\title{
LOW-ORDER MODELING OF THE UNSTEADY AERODYNAMICS IN FLAPPING WINGS
}

\author{
A Dissertation \\ Presented to the Faculty of the Graduate School \\ of Cornell University \\ in Partial Fulfillment of the Requirements for the Degree of \\ Doctor of Philosophy
}

\author{
by \\ Juan Carlos Gomez
}

August 2015 
(C) 2015 Juan Carlos Gomez

ALL RIGHTS RESERVED 


\section{LOW-ORDER MODELING OF THE UNSTEADY AERODYNAMICS IN FLAPPING WINGS Juan Carlos Gomez, Ph.D.}

Cornell University 2015

The investigation of unsteady aerodynamics is becoming a more attractive topic of research in enhancing flight capabilities. Natural flyers such as birds and insects can undergo flight maneuvers that are very difficult or not possible with current aerodynamic understanding. Modeling the unsteady phenomena produced by flapping wings is important to the understanding of these maneuvers, with possible applications to aircraft flight. We investigate reduced-order modeling of the unsteady aerodynamics generated by flapping wings using the two separate approaches of rotational lift and dynamic stall. A low order quasi steady model based on rotational lift and a revised version incorporating dynamic stall are presented. Both concepts are analyzed using simulated results, with experimental data measured with matching kinematics as a basis of comparison. This combined model is then used to conduct parametric studies of the time averaged aerodynamic forces over varying kinematic variables. 


\section{BIOGRAPHICAL SKETCH}

As a child of Colombian immigrants I grew up in a two bedroom government subsidized apartment with 7 relatives. My parents made very little money, but made the most of what they could provide. My childhood upbringing and education was characterized by many hurdles and roadblocks, but despite these economically and societally driven challenges I persevered to become the first in my family to achieve a college degree. In this early period of my life I developed the emotional courage, principles, and endurance to follow through with difficult tasks and to see the benefit of working for the greater good of society. It also gave me a very personal firsthand experience on the importance of educating others on the issues at the heart of the continued inequities and disparities in our society.

My undergraduate career at Rutgers University gave me my first opportunities to use my gifts and talents to broaden the educational exposure to underrepresented communities and to personally take responsibility for advocating for broader access to higher education. I began to gain a much deeper appreciation for why my parents had worked so long and hard to provide me and my sister with an education, despite their financial hardships: the realization that what you achieve in life is not defined by where you come from or where you are, but by your drive and commitment to achieve excellence.

Due to my family's financial situation, I chose to pursue a career in industry upon my graduation. I used this as an opportunity to broaden my experiences and gain valuable skills in the work place. The small size and rapid growth of the company allowed me to gain a wider array of responsibilities than what is typically associated with being a control systems engineer. As one of only three

minority members of a 50 member company I continued to work to broaden the 
conversation on the inequities and disparities in our society and to advocate for the spread of higher education. My time spent in industry, along with my previous undergraduate experiences, shaped and strengthened my desire to pursue a Ph.D. in aerospace engineering, not only for my intellectual pursuits, but also as a vehicle for greater outreach.

My graduate career has been a great personal struggle that in a way parallels my childhood challenges. I have struggled through self-doubt, alienation, and an inability to relate with many of my peers due to the drastically different background that I come from. Persevering through these hurdles I gained a renewed sense of emotional courage, principles, and endurance to follow through difficult times, as well as the importance of a dialogue on societal inequities. 
This document is dedicated to my adviser and mentor, the late Dr. Ephrahim Garcia. 


\section{ACKNOWLEDGEMENTS}

I would like to thank my fellow lab mates and colleagues for making my graduate career an interesting and engaging experience. I would also like to thank my friends and family for supporting me even through my most troubling times in academia. Special thanks go to my close friends in and outside of the engineering department and Cornell, whom there are too many to name here, for helping me retain my sanity through these times.

Most of all, I would like to thank Dr. Ephrahim Garcia. I thank him for believing in me when I did not even believe in myself, and, through a combination of incredible mentoring and sheer stubborness, being the only person to convince me to finish the doctoral program during my darkest moments. This

body of work could not have been done without him. For that, and for the past few years I had the pleasure of knowing him, I give him my greatest thanks. 


\section{TABLE OF CONTENTS}

Biographical Sketch . . . . . . . . . . . . . . . . iii

Dedication .......................

Acknowledgements . . . . . . . . . . . . . . . vi

Table of Contents . . . . . . . . . . . . . . . . . . vii

List of Figures $\ldots \ldots \ldots \ldots \ldots \ldots \ldots \ldots \ldots$ ix

1 Introduction 1

2 Low-Order Modeling of the Unsteady Aerodynamics in Flapping Wings

2.1 Nomenclature . . . . . . . . . . . . . . . . . 5

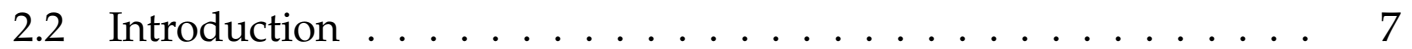

2.3 Modeling Approach . . . . . . . . . . . . . . . . . . . 12

2.3.1 Wing Kinematics . . . . . . . . . . . . . . . . . . . . . 13

2.3.2 Aerodynamic Modeling . . . . . . . . . . . . . . . . . . 16

2.4 Results and Discussion . . . . . . . . . . . . . . . . 20

2.4.1 Two Degrees of Freedom . . . . . . . . . . . . . . 23

2.4 .2 Three Degrees of Freedom . . . . . . . . . . . . . . . . . . . 29

2.4.3 Combining Models . . . . . . . . . . . . . . . . . . . . 31

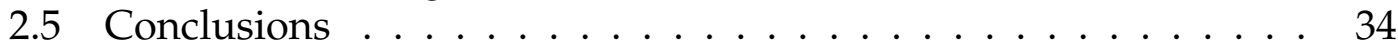

3 Parametric Study of the Unsteady Aerodynamics of Flapping Wings in Hover 36

3.1 Nomenclature . . . . . . . . . . . . . . . . . . . . . . . 36

3.2 Introduction . . . . . . . . . . . . . . . . 37

3.3 Modeling Approach $\ldots \ldots \ldots \ldots \ldots$

3.3.1 Wing Kinematics . . . . . . . . . . . . . . . . . . 40

3.3.2 Aerodynamic Modeling . . . . . . . . . . . . . . . . . . . 42

3.4 Results and Discussion . . . . . . . . . . . . . . . . . 44

3.4.1 Verification of Numerical Model . . . . . . . . . . . . . 44

3.4.2 Observations of Physical Mechanisms . . . . . . . . . . . . 49

3.4 .3 Frequency Effects . . . . . . . . . . . . . . . . 52

3.5 Conclusions . . . . . . . . . . . . . . . . . 53

4 Parametric Study of the Unsteady Aerodynamics of Flapping Wings in Forward Flight

4.1 Nomenclature . . . . . . . . . . . . . . . . . . . . . 55

4.2 Introduction . . . . . . . . . . . . . . . 56

4.3 Modeling Approach $\ldots \ldots \ldots \ldots \ldots$

4.3 .1 Wing Kinematics . . . . . . . . . . . . . . . . . . . . 59

4.3.2 Aerodynamic Modeling . . . . . . . . . . . . . . . 61

4.4 Results and Discussion . . . . . . . . . . . . . . . . . . . 64 
4.4.1 Verification of Numerical Model . . . . . . . . . . . . . . 64

4.4.2 Observations of Physical Mechanisms . . . . . . . . . . 70

4.4.3 Pitch and Stroke Amplitude Effects . . . . . . . . . . . 73

4.5 Conclusions . . . . . . . . . . . . . . . 75

$\begin{array}{lll}5 & \text { Conclusions } & 79\end{array}$

$\begin{array}{ll}\text { Bibliography } & 82\end{array}$ 


\section{LIST OF FIGURES}

2.1 Coordinate axes are shown in various views. Arrows point in the positive direction. . . . . . . . . . . . . . . . 13

2.2 Comparison of the lift curve amplitudes. Solid line comes from Kinsey and Dumas. Dashed line comes from the dynamic stall model with $\xi_{\text {nequiv }}=.85$ and $f_{n, \text { equiv }}^{\star}=0.14 \ldots \ldots \ldots \ldots$

2.3 The three waveforms chosen for the wing kinematics. Solid lines are the base waveforms. Rounded lines are the smoothed waveforms. . . . . . . . . . . . . . . . . . 21

2.4 Lift and drag profiles for $\Lambda=180^{\circ}, H=50^{\circ}$, and $\Lambda=180^{\circ}$, $H=50^{\circ} \ldots \ldots \ldots \ldots \ldots \ldots \ldots \ldots \ldots \ldots$

2.5 Forces due to unsteady terms, associated with Fig. 2.4 . . . . . 24

2.6 Lift and drag profiles for $\Lambda=60^{\circ}, H=0^{\circ}$, and $\Lambda=180^{\circ}, H=0^{\circ}$. 24

2.7 Forces due to unsteady terms, associated with Fig. 2.6. . . . . . . 25

2.8 Lift and drag profiles for $\Lambda=180^{\circ}, H=90^{\circ}$. . . . . . . . . 27

2.9 Component drag forces shared by both models. Left plot corresponds to Fig. 2.8. Right plot is a superposition of viscous forces

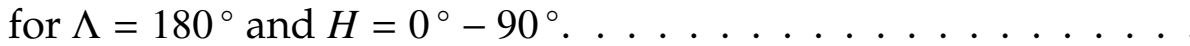

2.10 Simulations of the rotational lift and dynamic stall based models on the right, with Dickinson's results for comparison on the left [23]. A superposition of drag forces for $\Lambda=180^{\circ}$ and $H=0^{\circ}-90^{\circ} .28$

2.11 Lift and drag profiles for $\Lambda=180^{\circ}, H=45^{\circ}$, and $\Phi= \pm 25^{\circ}$. The frequency for $\phi(t)$ is set to the wingbeat frequency $f$. . . . . . . .

2.12 Lift and drag profiles for $\Lambda=180^{\circ}, H=45^{\circ}$, and $\Phi= \pm 25^{\circ}$. The frequency for $\phi(t)$ is set to twice the wingbeat frequency $f$. . . . .

2.13 Lift curves for 6 of the 8 two degree of freedom test cases. The dark solid line is the experimental data and the lighter dashed line is the combined model with appropriate values for the influence factors $s_{d}$ and $s_{r} \ldots \ldots \ldots \ldots \ldots \ldots \ldots$

3.1 Coordinate axes are shown in various views. Arrows point in the positive direction. . . . . . . . . . . . . . .

3.2 The three waveforms chosen for the wing kinematics. Solid lines are the base waveforms. Rounded lines are the smoothed waveforms. . . . . . . . . . . . . . . . . . 42

3.3 Stroke-averaged lift versus pitch amplitude. . . . . . . . . . . . 44

3.4 Experimental data minus forces not associated with Dynamic Stall and Rotational Lift. . . . . . . . . . . . . . . . . . . . . . 47

3.5 Stroke-averaged lift for $\Lambda=0^{\circ}-90^{\circ}$ and $H=0^{\circ}-90^{\circ}$. . . . . 50

3.6 Stroke-averaged lift for $0^{\circ}<\Lambda<90^{\circ}$ and $0^{\circ}<H<90^{\circ} \ldots \ldots \ldots 51$

3.7 Stroke-averaged lift for $f=0.1-1 \mathrm{~Hz} . \ldots \ldots \ldots \ldots$. . . . . 52

4.1 Coordinate axes are shown in various views. Arrows point in the positive direction. . . . . . . . . . . . . 
4.2 The three waveforms chosen for the wing kinematics. Solid lines are the base waveforms. Rounded lines are the smoothed wave-

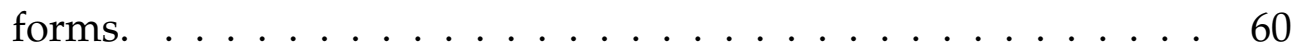

4.3 Lift and thrust for $\psi=45^{\circ}$ and $J=0.2 \ldots \ldots \ldots$. . . . . . 64

4.4 Instantaneous lift and thrust for $\psi=45^{\circ}$ and $J=0.47 \ldots \ldots . .65$

4.5 Experimental data minus forces not associated with Dynamic Stall and Rotational Lift for $J=0.2$. . . . . . . . . . . . . . 66

4.6 Experimental data minus forces not associated with Dynamic Stall and Rotational Lift for $J=0.47 \ldots \ldots \ldots$. . . . . . . . . 67

4.7 Stroke-averaged lift and thrust for all experimental data points. . 69

4.8 Stroke-averaged forces for $\psi=15^{\circ}-75^{\circ}$ and $J=0-0.6$. . . . . 70

4.9 Isolated contributions to stroke-averaged lift for $\psi=15^{\circ}-75^{\circ}$ and $J=0-0.6 \ldots \ldots \ldots \ldots \ldots \ldots \ldots \ldots \ldots \ldots \ldots \ldots \ldots$

4.10 Isolated contributions to stroke-averaged thrust for $\psi=15^{\circ}-75^{\circ}$

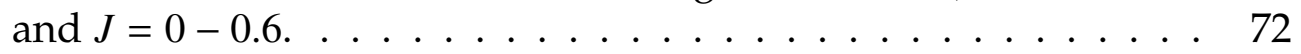

4.11 Peak stroke-averaged lift as pitch amplitude changes. . . . . . . . 74

4.12 Peak stroke-averaged thrust as pitch amplitude changes. . . . . . 74

4.13 Stroke averaged forces for $\Lambda=45^{\circ}$ and $H=10^{\circ}-90^{\circ}$. . . . . . 77

4.14 Stroke-averaged forces for $\Lambda=90^{\circ}$ and $H=10^{\circ}-90^{\circ}$. . . . . 78 
CHAPTER 1

\section{INTRODUCTION}

The unique attributes of unmanned aerial vehicles (UAVs) have driven their increased application in many areas. The commercial and personal uses that UAVs have begun to see range from point to point delivery service to recreational video and photography. They are also being applied to fulfill roles such as surveillance, reconnaissance, monitoring of disaster zones, and delivering medicine and supplies to remote areas. Their lower cost, increased ease of production, and mitigated risk to human life are some of the characteristics that make UAVs more appealing for these wide ranging applications, and has driven their increasing ubiquity in modern society.

Some of these applications may benefit from or even require capabilities, such as perching and hovering, that exist outside of the flight regime of fixed wing aircraft, but that we see birds and insects accomplish routinely. Because of this, another reason for the increased interest in UAVs is due to their potential flight capabilities.

Ornithopter flight, which is to say flapping wing flight, presents a host of unsteady aerodynamic phenomena that are not present in classical fixed wing aerodynamics. These phenomena are used by birds and insects to accomplish aerial feats that are either very difficult or not possible with conventional fixed wing aircraft, and, as such, are being studied for advancing current flight capabilities. The characteristics of UAVs mentioned before also make them ideal for experimental aerodynamics and flight research that involve these phenom-

ena. Morphing aircraft research such as wing shape change, re-orientation of tail and wing planform sections, and inflatable wings already make extensive 
use of UAVs [11].

Although classical aerodynamics, which deals with the steady fluid dynamics of fixed wings, has dominated for the greater majority of aviation history, interest in researching unsteady fluid phenomena has existed for almost a century. However, due to the popularity of fixed wing aircraft, ornithopter and unsteady fluid dynamics research waned for the majority of the $20^{\text {th }}$ century. More recently in the $21^{\text {st }}$ century, science and technology have reached a point where we can begin to look into taking advantage of unsteady aerodynamic effects to increase the efficiency and effectiveness of aerodynamic systems. This, in conjunction with the aforementioned increasing ubiquity of UAVs, has fueled the resurging focus on ornithopter and unsteady aerodynamics research.

The central role UAVs occupy is also guiding research efforts. The disparate applications UAVs face demand performance such as payload capacity, agility, and persistence in the field, objectives that vary depending on the application and situation. As a result, a need arises to perform design studies around flapping-wing UAVs to explore their capabilities and help guide the design of ornithopters optimized for specific applications.

A common approach for simulating fluid behaviour is to use CFD packages. The full 3D Navier Stokes equations are generally used, with appropriate simplification introduced that are problem dependent. However, due to the dynamic nature of flapping wings and complexity of the associated fluid dynamics, the simplifications required to make such problems tractable often render the solutions to be of little value. Even with the needed simplifications the computational costs tend to be too high for iterative algorithms such as in design and optimization studies. As such these are not often used in the study of the 
fluid dynamics of flapping wings.

Low-order numerical modeling, on the other hand, takes a different approach in tackling fluid dynamics problems. Low-order models take a phenomenological approach that is informed by the system being analyzed and focuses on only capturing the more important, governing aspects of a given problem. This inherently leads to singificantly more manageable computational costs when compared with the aforementioned approach. As such, a lowcomputational-cost, numerical framework is ideal for the purposes of design and optimization studies.

The impetus for this body of work is to evaluate the feasibility and applicability of low-order modeling techniques to the intersection between unsteady aerodynamics and ornithopter research. One of the cornerstones of the current study is the evaluation of how well a 2D low-order model formulated from limited $2 \mathrm{D}$ data can capture the salient features of the forces generated by a more complex system, namely 3D flapping wings. The other cornerstone is to demonstrate the potential of low-order models to characterize a large parameter space. These types of efforts will help enable future work in UAV design and optimization for specific roles and will help explore the capabilities of UAV ornithopters.

Chapter 2 focuses on analyzing the competing ideas of rotational lift and dynamic stall, two phenomena thought to be paramount in flapping wing flight. The comparison method involves numerically simulating a series of flapping wing experimental data sets. This analytical comparison is used to evaluate how well rotational lift and dynamic stall respectively capture the salient features of the forces generated by unsteady wing kinematics in three dimensions. The approach of treating them as seperate but supplementary concepts in the same 
model is also evaluated.

In chapter 3 we characterize the parametric space of an ornithopter in a hovering flight configuration using the low-order, combined model established in chapter 2 . The same experimental data is used to guide the choice of parametric space. We first investigate the stroke-averaged forces produced by the system and study how the combined model compares to experiment. We then utilize the combined model to characterize the parametric space and analyze how the stroke-averaged aerodynamic forces vary over the space.

Chapter 4 is the characterization of the parametric space of an ornithopter in a forward flight configuration. A different set of experimental data is used that describes forces generated by flapping wings configured for forward flight. In the same vein as chapter 2, here we investigate the stroke-averaged forces to study how the combined model compares to experiment and then utilize said model to characterize the parametric space and analyze how the strokeaveraged aerodynamic forces vary over said space. The capacity of the combined model is then evaluated by studying the behavior as we expand the boundaries of the parameter space.

This body of work is a starting point to move forward with greater understanding of how to better capture unsteady phenomena with low-order modeling techniques. It helps us in evaluating the approach of using existing data and techniques to model a more complicated system. In particular, it helps us gain a more intimate understanding of the applicability of the combined model, as well as its capacity as a simplified model utilizing limited finite data to model a more complex system. 
CHAPTER 2

LOW-ORDER MODELING OF THE UNSTEADY AERODYNAMICS IN

FLAPPING WINGS

\subsection{Nomenclature}

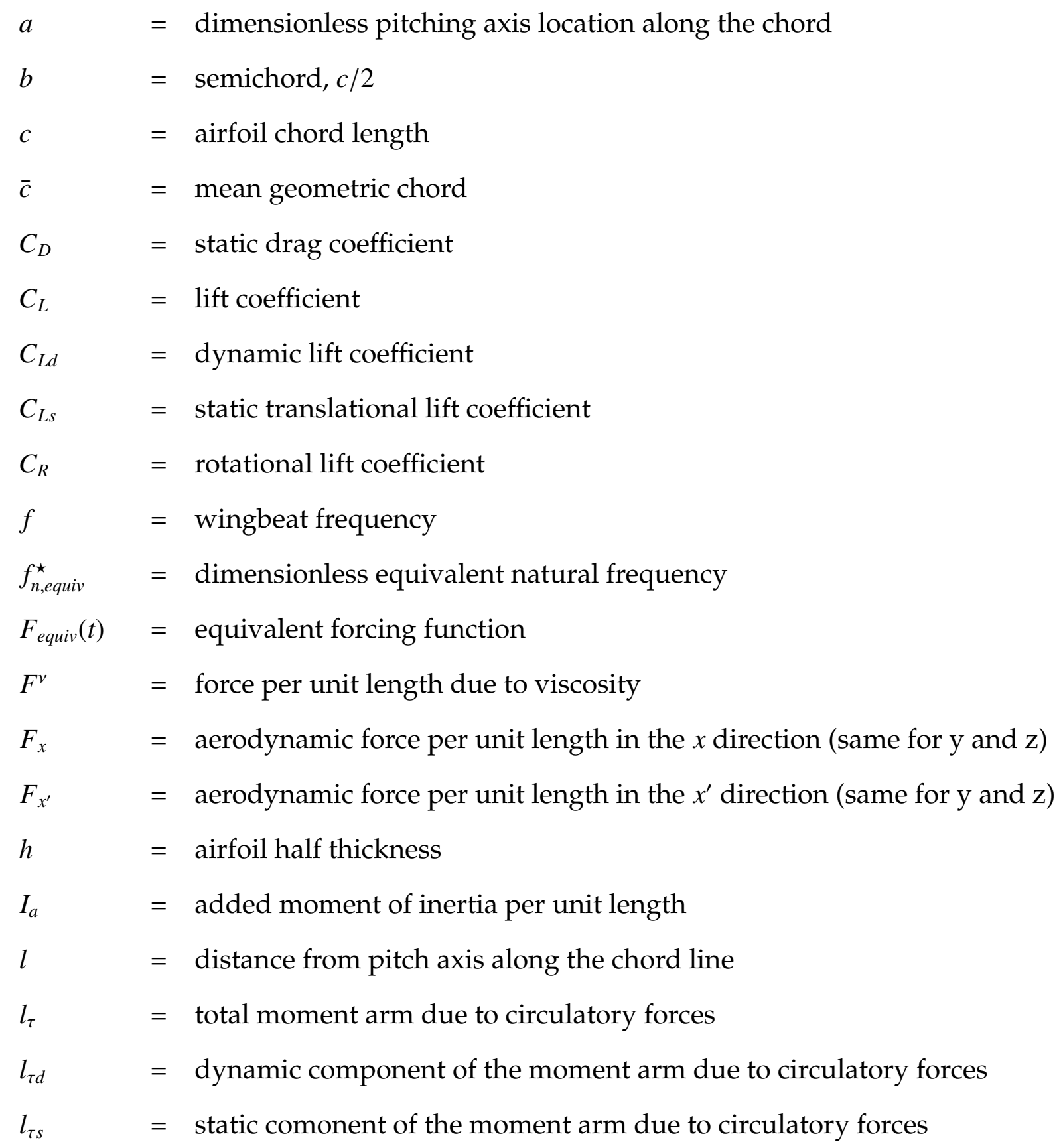




$$
\begin{array}{ll}
m & =\text { mass per unit length of one wing } \\
m_{11}, m_{22} & =\text { added mass terms, per unit length } \\
M_{p} & =\text { aerodynamic pitching moment per unit length acting about the pitch axis } \\
M^{v} & =\text { moment per unit length due to viscosity } \\
r & =\text { distance along the wingspan from the flapping rotation axis } \\
r_{w} & =\text { distance along the wingspan from the wing base } \\
R & =\text { span (length) of one wing } \\
s_{1}, s_{2}, s_{3} & =\text { dimensionless empirical coefficients of the dynamic stall model } \\
s_{d} & =\text { dynamic stall influence factor } \\
s_{r} & =\text { rotational lift influence factor } \\
t & =\text { time } \\
T_{v l} & =\text { vortex propogation timescale constant } \\
U_{c} & =\text { characteristic velocity } \\
U_{\infty} & =\text { freestream fluid velocity } \\
v_{n} & =\text { local velocity component normal to the arifoil chord line } \\
v_{x} & =\text { airfoil velocity component in the } x \text { direction (same for } \mathrm{y} \text { and } \mathrm{z} \text { ) } \\
v_{x^{\prime}} & =\text { airfoil velocity component in the } x^{\prime} \text { direction (same for } \mathrm{y} \text { and } \mathrm{z} \text { ) } \\
V_{x} & =\text { body velocity component in the } x \text { direction (same for } \mathrm{y} \text { and } \mathrm{z} \text { ) } \\
x_{c} & =\text { chordwise location from the leading edge } \\
\alpha & =\text { angle of attack } \\
\lambda(t) & =\text { sweep angle } \\
\Lambda & =\text { sweep amplitude } \\
\phi(t) & =\text { flapping angle } \\
\eta_{n} & =\text { flapping amplitude } \\
&
\end{array}
$$




$$
\begin{array}{ll}
\Gamma & =\text { circulation } \\
\rho_{\infty} & =\text { ambient fluid density } \\
\tau_{0} & =\text { flip start, expressed as fraction of wingbeat period } \\
\tau_{f} & =\text { flip duration midpoint, expressed as fraction of wingbeat period } \\
\tau_{v} & =\text { dimensionless vortex time } \\
\Delta \tau & =\text { flip duration, expressed as fraction of wingbeat period } \\
\omega & =\text { angular wingbeat frequency } \\
\omega_{n, \text { equiv }} & =\text { equivalent natural frequency } \\
\xi_{\text {equiv }} & =\text { equivalent damping ratio }
\end{array}
$$

\subsection{Introduction}

Ornithopter research has seen continually increasing interest in recent years. Science and technology have reached a point where we can begin to look into taking advantage of unsteady aerodynamic effects to increase the efficiency and effectiveness of aerodynamic systems. These advances also open the way to previously difficult or even unimaginable aircraft capabilities such as perching, hovering, and take-offs with no runway.

Although classical aerodynamics, which deals with the steady fluid dynamics of fixed wings, has dominated for the greater majority of aviation history, interest in researching unsteady fluid phenomena has existed for almost a century. These early endeavors are exemplified by Munk [16] who investigated the aerodynamic behavior of a wing during pitching, and Theodorsen [25] who studied aerodynamic instability and flutter, studies that are referenced for their scientific value to this day. However, due to the popularity of fixed wing air- 
craft, ornithopter and unsteady fluid dynamics research waned for the majority of the $20^{\text {th }}$ century.

Current ornithopter research is fueled by the increased interest and application of novel UAV and energy harvesting systems [11,4]. Unlike conventional fixed wing aircraft these systems inherently involve fluid dynamic behavior such as added mass and dynamic stall that are not captured by classical aerodynamic theory. As such, the need arises for modeling that can sufficiently capture the physical effects of these phenomena. Such models are required for furthering research into the capabilities of said aerodynamic systems through use in parametric and design optimization studies or in conjunction with other simulation tools such as structural models.

A common approach for simulating fluid behaviour is to use CFD modeling. The fundamental basis of almost all CFD problems are the full 3D Navier Stokes equations and the appropriate simplification of these equations. However, due to the dynamic nature of flapping wings and complexity of the associated fluid dynamics, the simplifications required to make such problems tractable tend to render the solutions so abstract as to not be of use for the types of studies previously described. Additionally, even with the needed simplifications the computational cost is too high for iterative algorithms such as in design and optimization studies.

An alternative approach, low-order numerical modeling, sacrifices the comprehensiveness and complexity of CFD simulations for simplicity and efficiency. Low-order models take a phenomenological approach and focus on only capturing the more important, governing aspects of a given problem or system which leads to much lower computational costs when compared with CFD simula- 
tions. With respect to unsteady aerodynamics these models quantify phenomena such as added mass, circulatory effects, and viscous effects that we see from physical experimentation by applying established theory and developing semiempirical terms based on available experimental data $[6,34,35]$.

In 1993 DeLaurier proposed applying a low-order analytical model to predict the forces on a flapping wing ornithopter [5]. This was the first application of low-order modeling techniques to simulating the forces generated by flapping wings. The impetus for this development was to calculate the lift and drag generated by a physical pterosaur model being built by Aerovironment for the National Air and Space Museum. This phenomenological model attempts to quantify some of the physical effects seen in flapping flight: circulation derived lift and drag, added mass, leading edge suction, and viscous effects through the use of seperate mathematical terms that encompass each phenomenon.

Another phenomenological model has been developed by Wang et al. [1]. This low-order model quantifies circulatory effects, viscosity, and added mass. A key feature is the treatment of the circulatory effects as separate translational and rotational terms. This particular model was initially developed to predict aerodynamic behavior at Reynolds numbers on the order of $10^{2}-10^{3}$. Whereas Delaurier modeled a Pterosaur with an 18ft wingspan, Wang's model was first developed to simulate falling cards and subsequently used for numerical simulations of insect flight [3].

Alternatively, Dickinson has performed detailed experimental analysis on a mechanical model of hawkmoth wings [6]. This is a motor powered, scaled mechanical model submerged in a mineral oil tank. This research consisted of multiple experimental runs with varying flapping and pitching amplitudes. The 
analysis quantifies aerodynamic characteristics such as translational induced lift, rotational induced lift, and wake capture. Particular emphasis was placed on how these phenomena change as the amplitude is varied. Such studies help to better inform the development of semi-empirical terms used in low-order aerodynamic models.

Ellington conducted similar research with a mechanical wing model, but with an additional experimental component with live hawkmoths for comparison [9]. This involved analysis of the flow visualization around both sets of experiments, which was accomplished with strategically placed smoke. The various vortices produced by the flapping motion and their time varying structures were tracked and analyzed. This visualization showed that dynamic stall takes a central role in producing these shed vortices.

More recent work involves numerical studies of canonical pitch-up and pitch-down wing maneuvers for varying cross-sections with companion experimental work $[20,7,19]$. These studies are formulated in such a way so as to tease out the relationship between varying aspects of wing kinematics and fluid phenomena, as well as the influence dimensionless quantities such as Reynolds number, reduced frequency, and Strouhal number have on phenomenon behavior.

With the insights gained through these research groups low-order models have matured and developed to more accurately reflect what is physically observed [13, 27]. An example of this evolution is Bryant et al.'s modified version of Wang's model [4]. As opposed to rotational lift, the work of Bryant et al. involves modifying the circulation coefficients to incorporate the effects of an unsteady phenomenon known as dynamic stall, a concept also used by Wick- 
enheiser and Garcia to model aircraft in perching maneuvers [32]. These modifications are in the same vein as those used by the ONERA model and involve expanding the circulatory lift coefficient into a static term and a dynamic term, with the dynamic term expanded into an ordinary differential equation with empirical coefficients of its own [26]. This dynamic stall approach was shown to have good results in the energy harvesting regime when compared to CFD simulations of a 2D pitching and heaving airfoil done by Kinsey and Dumas [14].

Certain studies have shown that the forces seen in flapping wings once thought to be solely due to rotational lift may also be related to dynamic stall effects. As in Wang's model, a prevailing school of thought for flapping wing aerodynamics separates the forces due to circulation into a static translational component and a rotational component, where the rotational component attempts to capture the phenomena not accounted for in the static component. Research by Walker shows that the unsteady circulatory forces that arise when rotation is introduced to wing motion may be of a more complicated nature than simply being due to the rotation itself and instead may be related to the vortices produced by such motion [29]. This insight is one of the motivating factors for the dynamic stall approach taken by Bryant et al. and introduces an alternative school of thought to rotational lift. Work such as that by Bryant's group also brings up the question of how well the two competing ideas capture unsteady effects and what aspects they may respectively encompass, a central focus of the current work.

The impetus for the present study is to evaluate the feasibility of utilizing low-order modeling within a greater investigative framework. The simplicity 
and low computational cost is ideal for integration into the types of iterative and multi-tiered numerical frameworks such as for example the ones used in design and optimization studies. However it is important, and the purpose of the current study, to evaluate how well a 2D low-order model formulated from limited 2D data can capture the salient features of the forces generated by 3D flapping wings.

This paper focuses on analyzing the competing ideas of rotational lift and dynamic stall. The comparison method involves numerically simulating a series of experimental tests conducted by Sane and Dickinson [23], which involved gathering force data across a range of wing kinematics in a Reynolds number regime between $10^{2}$ and $10^{3}$. This framework is used to explore the behavior of both concepts against real world unsteady aerodynamic behavior.

Here we evaluate how well rotational lift and dynamic stall respectively capture the salient features of the forces generated by unsteady wing kinematics in three dimensions, as well as evaluating the idea of treating them as seperate but supplementary concepts in the same model. This analysis is a starting point to move forward with greater understanding of how to better capture unsteady phenomena with low-order modeling techniques. It also helps us in evaluating the approach of using existing data and techniques to model a more complicated system.

\subsection{Modeling Approach}

The numerical models used in this paper are described here. Because both use the same basis phenomenological formulation there are certain characteristics 
common to both. Of these similarities it is worth noting that in their original forms these models are used for 2D calculation and analysis. As such, the theory and mathematics involved require some extension into the 3 dimensional realm. This is most evident in the wing kinematics section.

\subsubsection{Wing Kinematics}

We seek to model three-dimensional wing motion. Here we describe this by defining three degrees of freedom: the flapping angle $\phi(t)$, pitch angle $\eta(t)$, and sweep angle $\lambda(t)$. It should be noted that most engineering models only consider two degrees of wing freedom, heaving (flapping) and pitching, but we have extended our model to include sweep as our third degree of freedom.

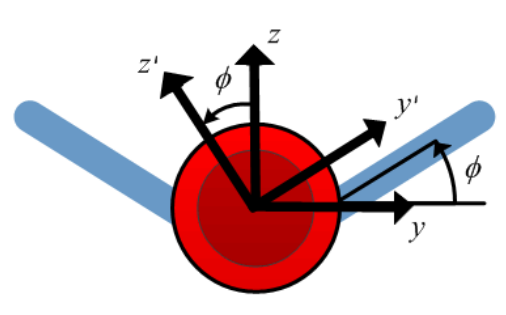

(a) Front view

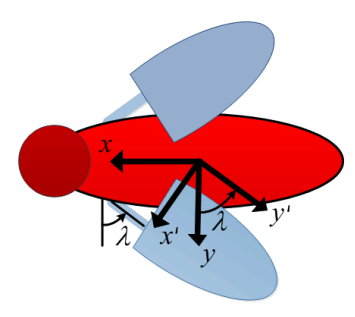

(b) Top view

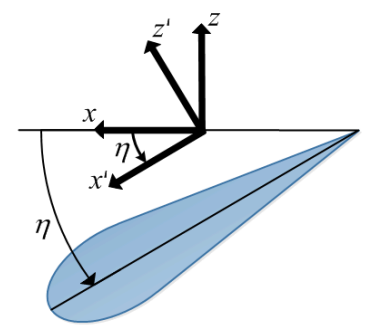

(c) Airfoil-centered view

Figure 2.1: Coordinate axes are shown in various views. Arrows point in the positive direction.

As can be seen in Fig. 2.1 these angles define the relationship between the three-dimensional vector orientation of a body-centered (unprimed) coordinate system and a coinciding coordinate system corotating with an airfoil section (primed), with a separate corotating system associated with each 2D airfoil section used in our three-dimensional approximation. The corotating systems realign themselves with the airfoil sections at each instance in time, but do not 
translate with them, instead having their origins always coincide with the origin of the body-centered system. These $x-y-z$ systems are defined in such a way as to preserve the right hand rule, with the directions shown in Fig. 2.1 being positive.

Because of the three-dimensional nature of the wing motion we require a clear and compact way of expressing the corresponding equations of motion. To accomplish this we use rotation matrices to mathematically describe our three degrees of freedom. Our defined rotations can be thought of as similar to simple gimbal rotations where each one is about a primary axis. This leads us to express the corresponding rotation matrices as

$R_{\lambda}(t)=\left[\begin{array}{ccc}\cos (\lambda) & \sin (\lambda) & 0 \\ -\sin (\lambda) & \cos (\lambda) & 0 \\ 0 & 0 & 1\end{array}\right], \quad R_{\phi}(t)=\left[\begin{array}{ccc}1 & 0 & 0 \\ 0 & \cos (\phi) & \sin (\phi) \\ 0 & -\sin (\phi) & \cos (\phi)\end{array}\right], \quad R_{\eta}(t)=\left[\begin{array}{ccc}\cos (\eta) & 0 & -\sin (\eta) \\ 0 & 1 & 0 \\ \sin (\eta) & 0 & \cos (\eta)\end{array}\right]$

where the element containing 1 is associated with the axis of rotation of that degree of freedom. Because we assume a rigid wing $\phi(t), \lambda(t)$, and $\eta(t)$ do not vary along the span and are not functions of $r$, the local distance from the shoulder joint or rotation axis. As a consequence the relation between the body-centered system and each airfoil-corotating system simplifies to the same mathematical expression. This allows us to characterize the velocities along the span of the wing in the compact form

$$
\underline{v}_{w}^{\prime}=R_{\eta}\left[R_{\phi}\left[R_{\lambda} \underline{v}_{b}+A_{\lambda}\right]+A_{\phi}\right]
$$

Here $\underline{v}_{b}$ and $\underline{v}_{w}^{\prime}$ are the velocity vectors measured in the inertial frame and are 
defined as

$$
\underline{v}_{b}(t)=\left[\begin{array}{c}
V_{x} \\
V_{y} \\
V_{z}
\end{array}\right], \quad \underline{v}_{w}^{\prime}(t, r)=\left[\begin{array}{l}
v_{x^{\prime}} \\
v_{y^{\prime}} \\
v_{z^{\prime}}
\end{array}\right]
$$

where $\underline{v}_{b}(t)$ contains the velocities associated with the ornithopter body oriented with the body-centered system and $\underline{v}_{w}^{\prime}(t, r)$ the airfoil velocities oriented with the airfoil-corotating system. As can be seen in Fig. 2.1 the elements $\left\langle V_{x}, V_{y}, V_{z}\right\rangle$ are associated with the $\langle x, y, z\rangle$ directions and $\left\langle v_{x^{\prime}}, v_{y^{\prime}}, v_{z^{\prime}}\right\rangle$ with the $\left\langle x^{\prime}, y^{\prime}, z^{\prime}\right\rangle$ directions, respectively. The terms $A_{\lambda}$ and $A_{\phi}$ contain elements that vary along the span, which are the tangential velocity components due to rotation about a point. These are expressed as

$$
A_{\lambda}(t, r)=\left[\begin{array}{c}
r \dot{\lambda} \\
0 \\
0
\end{array}\right], \quad A_{\phi}(t, r)=\left[\begin{array}{c}
0 \\
0 \\
r \dot{\phi}
\end{array}\right]
$$

Because the airfoil velocity is taken from the pitching pivot point, $\eta$ affects orientation but not velocity.

Assembling the airfoil velocity vectors $\underline{v}_{w}^{\prime}(t, r)$ associated with each airfoil section gives us the velocity profile of the entire wing. It should be noted that $\underline{\dot{v}}_{w}^{\prime}$ is with respect to the body reference frame, but expressed in the airfoilcorotating coordinate system. With this clarification and the convention chosen for our coordinate systems we can properly define our angle of attack as

$$
\alpha(t, r)=-\tan ^{-1}\left(\frac{v_{z^{\prime}}}{v_{x^{\prime}}}\right)
$$




\subsubsection{Aerodynamic Modeling}

We begin with Anderson, Pesavento, and Wang's rotational lift based aerodynamic model [1]. The fundamental purpose of this model is to predict the aerodynamic forces generated by an airfoil that is simultaneously heaving (translating) and pitching (rotating) in two dimensions. Here we assume the aerodynamic forces arise from two seperate sources: translational (circulatory) lift $C_{L s}$ generated by heaving and rotational lift $C_{R}$ generated by pitching.

In its original incarnation this model assumed rotation about the $\frac{1}{2}$ chord, however here we use a modified version that has been generalized for rotation about any point along the airfoil chord line.

The second aerodynamic model we use in our study is the dynamic stall model by Bryant, Gomez, and Garcia. The dynamic stall model foregoes rotational lift for dynamic stall effects. Dynamic stall arises from leading edge vortices (LEVs) that temporarily increase lift properties while they remain attached to the upper wing surface. This focus on modeling LEVs, as opposed to TEVs (trailing edge vortices), is supported by research that indicates LEVs are the dominant influencing vortical structure in the forces generated by pitching and plunging $[7,19]$. For an in-depth discussion on this dynamic stall model, as well as the generalized rotational lift model, please see Bryant, Gomez, and Garcia [4].

We must set appropriate values for the constants of the dynamic stall ODE. To facilitate this we first define an appropriate characteristic velocity $U_{c}$ and static translational lift coefficient $C_{L s}$. For $U_{c}$ we look to the work done by Shkarayev and Silin for insight and define our characteristic velocity as 


$$
U_{c}=\sqrt{\left(U_{\infty}\right)^{2}+(2 R f \Lambda)^{2}}
$$

where $2 R f \Lambda$ is the stroke averaged wingtip speed based on the sweep amplitude $\Lambda$ [24]. This formulation simplifies to the freestream fluid velocity (relative to the ornithopter body) $U_{\infty}$ at the limit of fixed wing forward motion and to $2 R f \Lambda$ at the limit of hovering ornithopter flight.

The equation for the static translational lift coefficient $C_{L s}$ we use here is that derived by Dickinson, Lehmann, and Sane for a scaled mechanical model of a fruit fly [6]. This was found by fitting a curve to experimental data gained through static wind tunnel testing of their three dimensional mechanical model. The equations for both $C_{L s}$ and the static drag coefficient $C_{D}$ are given as

$$
\begin{gathered}
C_{L s}=.225+1.58 \sin \left(2.13 \alpha-7.2 \frac{\pi}{180}\right) \\
C_{D}=1.92-1.55 \cos \left(2.04 \alpha-9.82 \frac{\pi}{180}\right)
\end{gathered}
$$

where everything within the sine and cosine functions are taken to be in radians. These equations are used in both the dynamic stall and rotational lift based models.

Inherent to 3D wing motion are the effects of unsteady tip vortices and finite span effects. Due to their nature they are out of the scope of 2D modeling. However with respect to this Eqs. (2.7) and (2.8) serve another purpose: as already mentioned these equations are derived from experimental data taken of a three dimensional wing and as such they encompass the effects of tip vortices and finite span under steady conditions. This is used in our model, as opposed 
to Bryant's original model where he uses data based on 2D simulations, as a first approximation to the tip vortex and finite span effects under flapping wing conditions.

With our characteristic variables defined we now look to properly tune our dynamic stall ODE constants. Appropriate relevant data is scarce. However studies by Eldredge, Wang, and OL have concluded that wing cross section has only a minor influence on the forces produced $[7,19]$, with other visualization work showing only fine feature differences in the vortices generated [20]. There has also been a plethora of work done across a number of Reynolds number ranges indicating a certain level of Re insensitivity with respect to LEV formation. In particular, very slight differences from Re 100-1000, and almost indistinguishable force and LEV formation from Re 2000-10000 [20].

With these insights on the insensitivities to wing cross section and Reynolds number, and due to a lack of available data to guide us, we follow Bryant et al. and use the CFD parametric study of Kinsey and Dumas [14]. This data set was produced for a 2D NACA0015 airfoil undergoing 2D motion consisting of pitching and heaving at a Reynolds number of 1100. Despite only representing a small range of simplified kinematics, we still take this to be a reasonable initial set of criteria for our parameter tuning. 


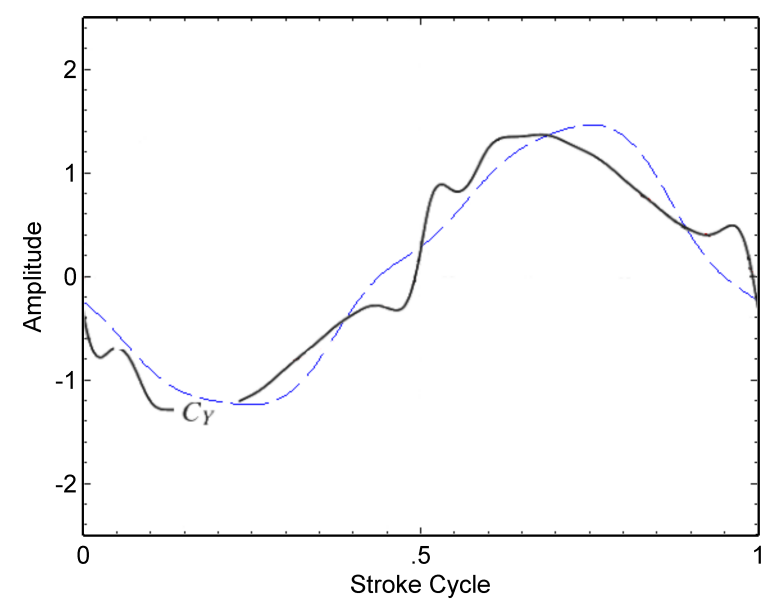

Figure 2.2: Comparison of the lift curve amplitudes. Solid line comes from Kinsey and Dumas. Dashed line comes from the dynamic stall model with $\xi_{n, \text { equiv }}=.85$ and $f_{n, \text { equiv }}^{\star}=0.14$.

The CFD data shows a peak in dynamic stall effects around a dimensionless frequency of 0.14 . We interpret this to be a sort of dynamic stall 'resonant frequency', which we in turn take as being equivalent to our dimensionless natural frequency $f_{n, \text { equiv }}^{\star}$. We tune our damping ratio $\xi_{n \text {,equiv }}$ so that our lift curve amplitudes approximate those given in Fig. 5 of reference [14], reproduced here in Fig. 2.2. This gives us $\xi_{n, \text { equiv }}=.85$.

Although LEVs are considered the main contributor to force augmentation, in three dimensional flow other structures such as tip vortices and TEVs also exist. CFD simulations have shown these phenomena to interact and merge with each other in varying ways to form very complex flow structures around the wing [28]. The modeling of such a system is a daunting task and out of the scope of current work. However, finite span effects are taken into consideration as previously described.

The blade element approach is used to extend both the rotational lift and 
dynamics stall models to three dimensions, where the forces are now calculated for multiple 2D airfoil cross sections and then integrated over the span of the wing.

\subsection{Results and Discussion}

Whereas Bryant used the dynamic stall model in a two dimensional energy extraction regime, here we wish to explore the behavior of both models under three dimensional conditions that produce lift and thrust. To accomplish this a set of experimental data gathered by Sane and Dickinson has been chosen as the basis for our simulations [23]. The physical parameters of our simulation are chosen such that it matches the Reynolds number, based on the sweep amplitude, of Dickinson's experiments which is on the order $10^{2}$, in the range of insect flight. Although the exact value varies with the kinematics of each experiment, it does remain within the appropriate range. As done in the original experimental study we take the wing geometry to be that of the Drosophila melanogaster (fruit fly) and match the associated dimensions to Dickinson's robofly testing platform.

To facilitate a biomimetic motion, as well as for the sake of comparative analysis, we are interested in the three waveforms shown in Fig. 2.3: the sine, trian-

gle, and trapezoidal waveforms for the heaving, sweep, and pitch, respectively. When combined these waveforms can produce patterns that roughly approximate the wing patterns of various insects including the fruit fly $[8,33]$.

To fully define the trapezoidal waveform we prescribe a flip duration $\Delta \tau$, the time it takes for the wing to flip, and a flip start $\tau_{0}$, the point in time that the flip 

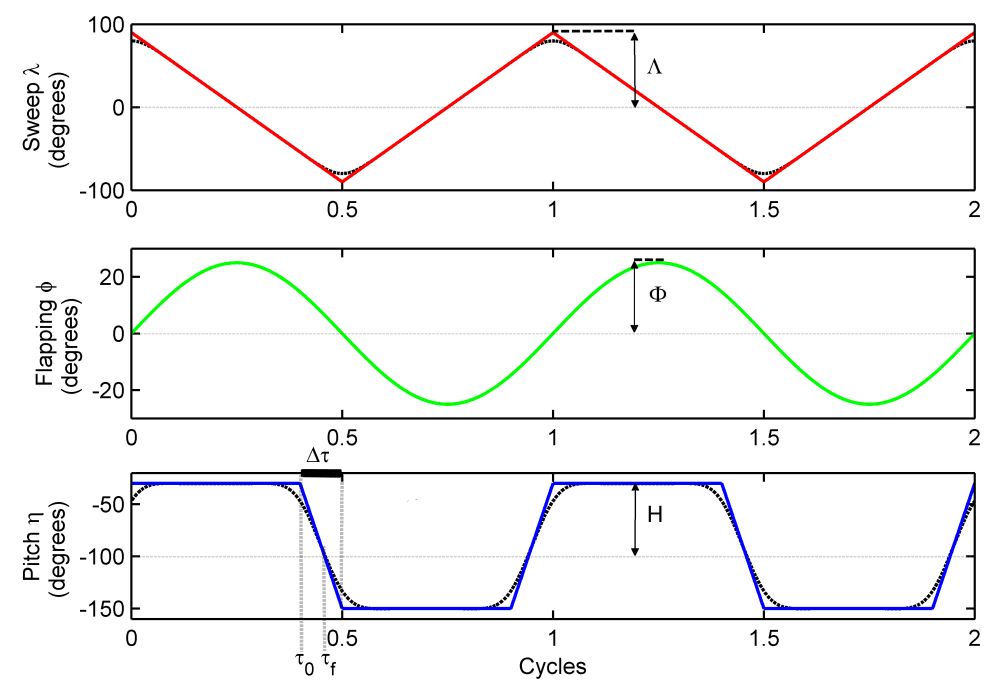

Figure 2.3: The three waveforms chosen for the wing kinematics. Solid lines are the base waveforms. Rounded lines are the smoothed waveforms.

begins, with both expressed as a fraction of wingbeat period. With these two variables we can also define the following useful quantity

$$
\tau_{f}=\tau_{0}+0.5 \Delta \tau
$$

where $\tau_{f}$ is the midpoint of the flip period, also expressed as a fraction of wingbeat period. We can now fully prescribe where and for how long the flip occurs during the stroke cycle.

The final piece needed to properly define representative wing kinematics is smoothing of the triangle and trapezoidal waveforms. Seperate functions have been devised to produce smooth versions of these waveforms such as the Eldredge function [7], however to gaurantee the same processing of the trapezoidal and triangular waveforms a $4^{\text {th }}$ order low-pass Bessel filter with a $50 \mathrm{~Hz}$ cutoff frequency was employed with representative results shown in Fig. 2.3 as the rounded lines. Here we have smoothed the sharp corners of our waveforms 
giving us derivatives that are well behaved at and around the corners, and motion that is more akin to insect wing kinematics [8]. A cutoff frequency of $50 \mathrm{~Hz}$ was chosen in order to retain the salient features of the waveforms, especially those associated with the flip period which tends to become easily obfuscated.

In addition to the experimental results Sane and Dickinson [23] also calculate the lift and drag predicted by the curve fitting to 3D static aerodynamic empirical data given by Eqs. (2.7) and (2.8). With the experimental data and static prediction we have a framework with which to analyze the behavior and nature of both models. For the sake of maximizing clarity in our comparisons, using editing software we have constructed from scratch figures that resemble those found in Dickinsons referenced work [23] and have extracted their profiles and overlayed them directly onto our plots.

We first look at the aerodynamic force behavior under a simplified set of kinematics involving only $\lambda(t)$ and $\eta(t)$, and then extend our analysis to kinematics involving all three degrees of freedom. For all of the following figures the solid black line is the experimental data, dotted black line static force estimates (ignoring all unsteady effects), lighter dashed line the rotational lift model, and lighter dot-dashed line the dynamic stall model. 


\subsubsection{Two Degrees of Freedom}

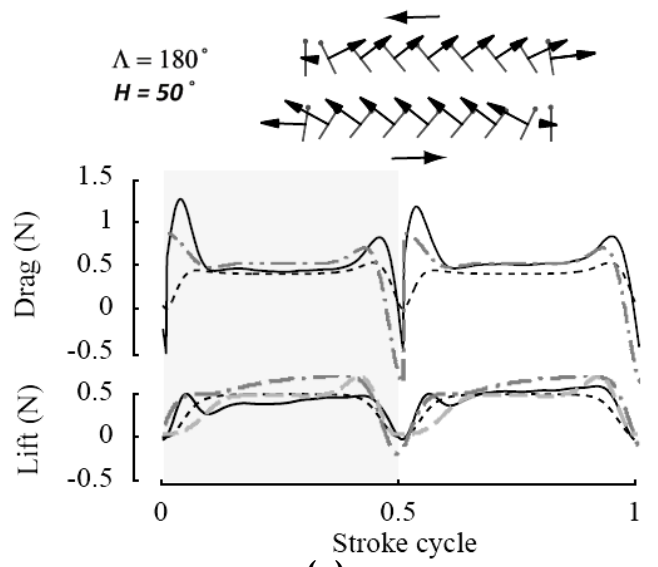

(a)

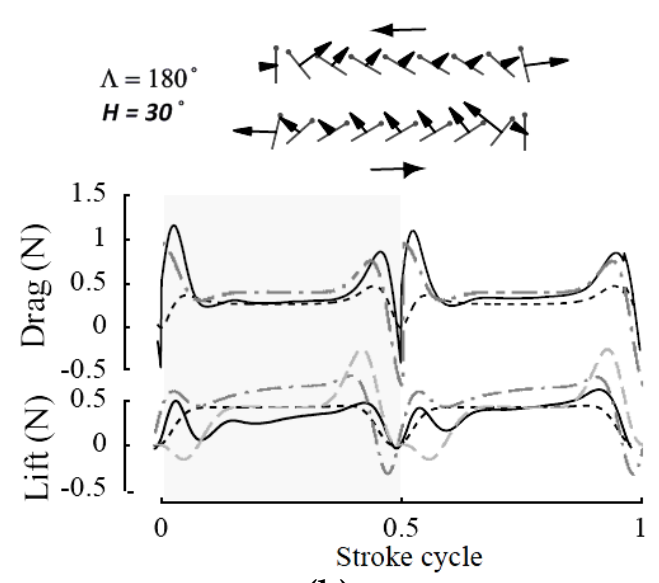

(b)

Figure 2.4: Lift and drag profiles for $\Lambda=180^{\circ}, H=50^{\circ}$, and $\Lambda=180^{\circ}$, $H=50^{\circ}$.

Using the experimental data curves in Sane and Dickinson's work as a guide we can see interesting differences in the mid-cycle behavior between the two models in Fig. 2.4, pertaining to $\Lambda=180^{\circ}$ and $H=50^{\circ}, 30^{\circ}$. During the midcycle (the time period between the half-cycle points $0,0.5$, and 1 ) the static force prediction gives a constant force, completely missing the time varying features of the experimental data. The rotational lift based model gives a constant force during the mid-cycle that is very similar to the static force prediction, also missing the salient features of the experimental data. The dynamic stall based model is the only one to give a time varying force curve that closely resembles the experimental data, albeit with slightly larger magnitudes.

These differing behaviors are due to the differences in the influencing factors of the rotational lift and dynamic stall terms. The rotational lift term is dependent on the instantaneous pitch rate $\dot{\eta}(t)$, which for these kinematics peaks around the half-cycle and goes to zero during the mid-cycle. The dynamic stall 


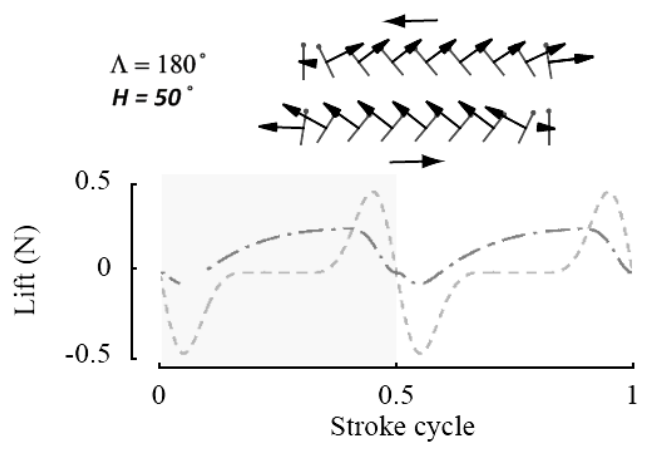

(a)

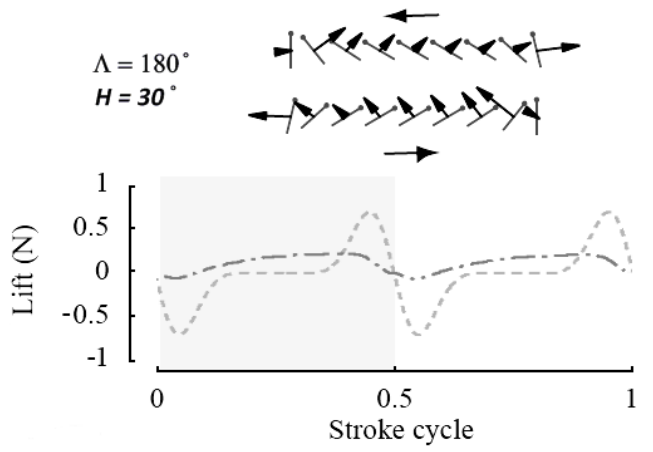

(b)

Figure 2.5: Forces due to unsteady terms, associated with Fig. 2.4.

model on the other hand utilizes an ordinary differential equation, giving us a curve that is affected by the behavior at the half-cycle points as well. This can more clearly be seen in Fig. 2.5 where we have isolated the force components due to rotational lift and dynamic stall. Here we see the force due to rotational lift drop to zero during the mid-cycle where $\dot{\eta}(t)$ also drops to zero, and the force due to dynamic stall take on a varying non-zero value which when combined with the other force terms more closely resembles the experimental lift force curves.

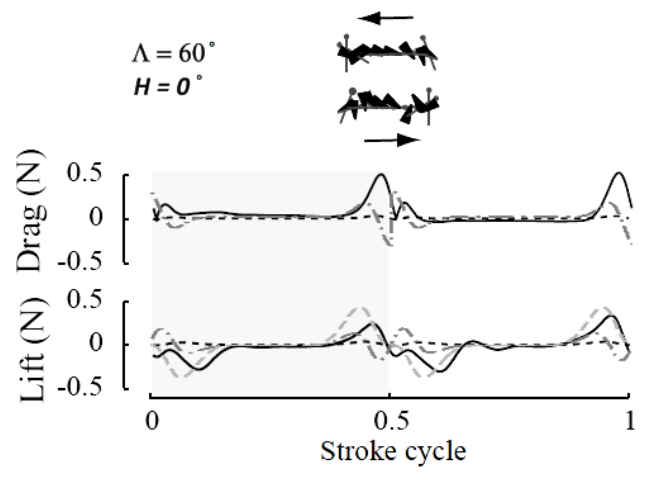

(a)

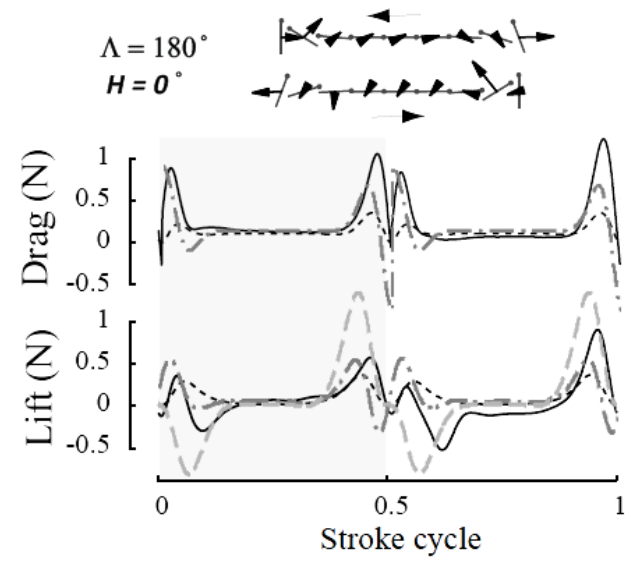

(b)

Figure 2.6: Lift and drag profiles for $\Lambda=60^{\circ}, H=0^{\circ}$, and $\Lambda=180^{\circ}$, $H=0^{\circ}$. 
We can shed more light on the half-cycle behavior of our models through the analysis of Fig. 2.6, pertaining to $\Lambda=60^{\circ}, 180^{\circ}$ and $H=0^{\circ}$. In these simulations the half-cycle behavior is more pronounced and thus more easily observed and analyzed, since the angle of attack of the wing is zero except at stroke reversal. This is due to the wing experiencing greater accelerations around the half-cycle points which is caused by the pitch angle flipping $180^{\circ}$ and completely reversing orientation around that point in the stroke cycle.

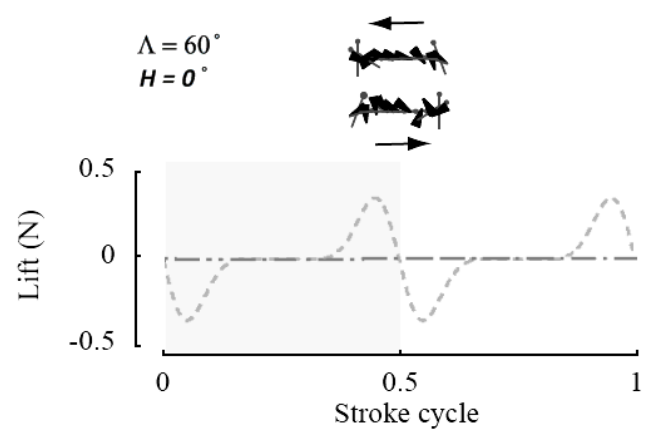

(a)

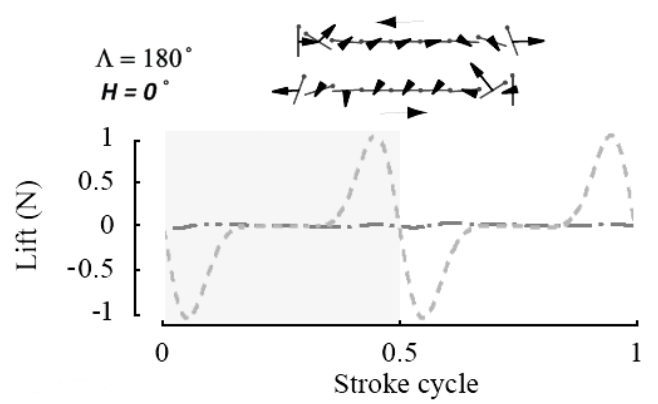

(b)

Figure 2.7: Forces due to unsteady terms, associated with Fig. 2.6.

The force curves in Fig. 2.6 show discrepancies between all models and experimental data. The shape of the curves corresponding to the rotational lift model appear to more closely resemble that of the experimental data, however the peaks and troughs around the half-cycle points are being considerably overestimated. The dynamic stall model curves exhibit a scaling similar to the experimental data, but the peaks that appear right before and troughs that appear after the half cycle are being underestimated.

The rotational lift and dynamic stall behaviors can more clearly be seen in Fig. 2.7, where we have once again isolated them from the rest of the terms in our phenomenological models. Significant spikes can be seen around the 
half-cycle for rotational lift, but no significant behavior is observed for the force curve pertaining to dynamic stall. We again see that the salient features of the rotational lift based force curves fall in the area of the stroke cycle where pitch rate $\dot{\eta}(t)$ occurs, having its greatest value in Fig. 2.6, and falls off where $\dot{\eta}(t)$ goes to zero. This in conjunction with our observation of Fig. 2.4 shows that the dependence on $\dot{\eta}(t)$ is one of the primary factors for the salient features of the rotational lift based model, but also the cause of the overestimation. The dynamic stall model on the other hand does not appear to be properly resolving the underlying phenomenological mechanism causing the peaks and troughs around the half-cycle. Gaining a better grasp of the source of these discrepencies will require further investigation in the future.

A characteristic that is particular to the test cases involving only two degrees of freedom is the drag force being exactly the same for both models, thus choosing to only show the dynamical stall line in the corresponding drag plots. This is due to having no freestream fluid velocity and $\phi(t)$ being held at $0^{\circ}$, which causes the angle of attack $\alpha$ and pitch $\eta$ to be the same. If we look back at our aerodynamic models, the rotational lift coefficient $C_{R}$ and dynamic stall coefficient $C_{L d}$ only appear in terms that will affect the force coefficient $C_{L}$, which due to $\alpha$ and $\eta$ being the same is applied purely in the $z$ direction. Because we define lift to be in the $z$ direction (vertical) and drag to always be parallel to the direction of motion of the airfoil, which in this case is always horizontal (in the sweeping plane) and perpendicular to the lift direction, the effects of both rotational lift and dynamic stall will be isolated to the $z$ direction.

There is however a striking behavioral trend to gather from analyzing the drag data. The trend stems from a discrepancy between the experimentally 
produced drag forces and those predicted by both reduced-order models. This phenomenon is evident when $\Lambda=180^{\circ}$ and is most pronounced in Fig. 2.8. Here we can see a significant peak in the drag post half-cycle that is not observed in the model derived drag curves.

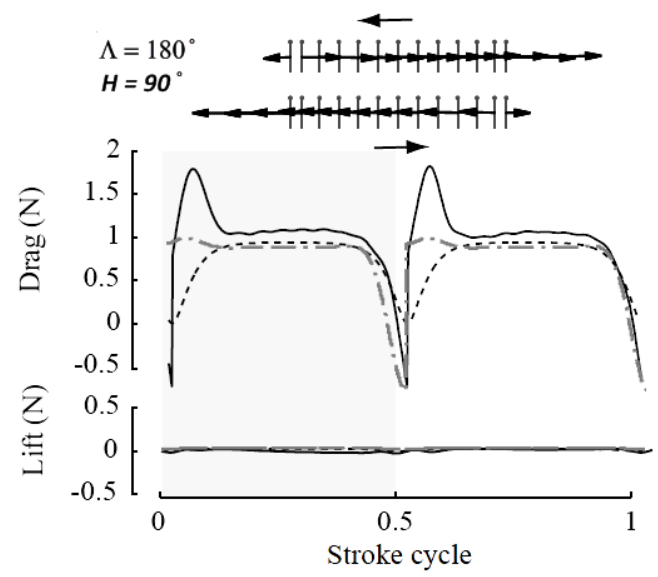

Figure 2.8: Lift and drag profiles for $\Lambda=180^{\circ}, H=90^{\circ}$.

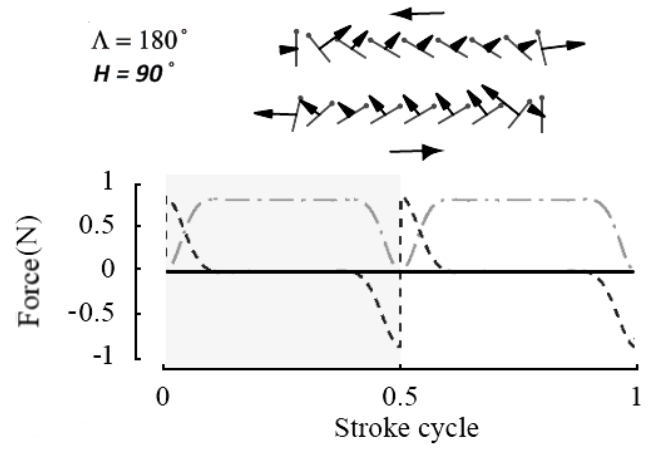

(a)

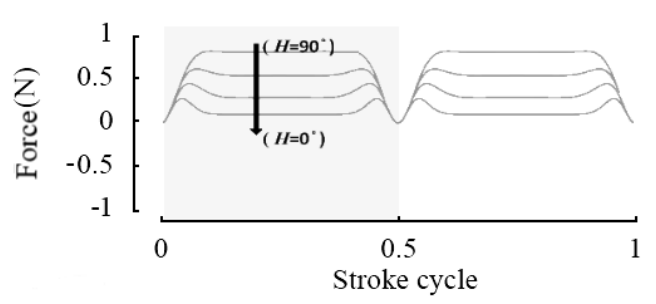

(b)

Figure 2.9: Component drag forces shared by both models. Left plot corresponds to Fig. 2.8. Right plot is a superposition of viscous forces for $\Lambda=180^{\circ}$ and $H=0^{\circ}-90^{\circ}$.

As explained earlier the effects of rotational lift and dynamic stall for these sets of kinematics are isolated to the $z$ direction and do not contribute to the drag, implying that the unsteady terms in our models have no contribution to these post half-cycle peaks in the drag curves. The left plot in Fig. 2.9 shows the 
individual drag force components involved, with only added mass and the force due to viscosity having significant contributions. Of these two only the viscous force undergoes any significant change when the pitching amplitude $H$ is varied between $0^{\circ}$ and $90^{\circ}$. The right plot of Fig. 2.9 shows the variations in the viscous force across this variation in $H$. When comparing these curves with those of the experimentally produced force curves we can see that the viscous force cannot be responsible for the post half-cycle peaks, indicating the possibility of another mechanism being involved.

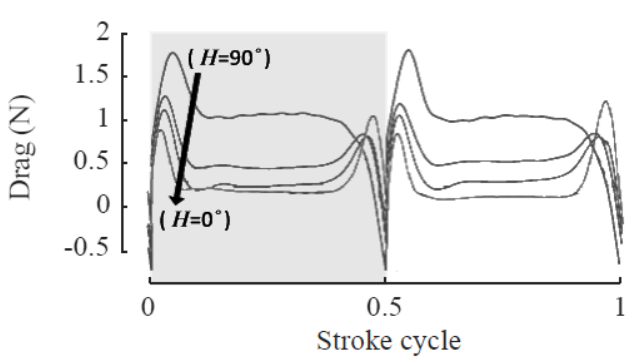

(a)

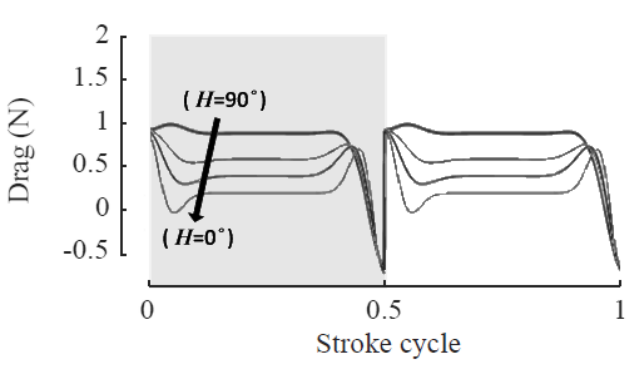

(b)

Figure 2.10: Simulations of the rotational lift and dynamic stall based models on the right, with Dickinson's results for comparison on the left [23]. A superposition of drag forces for $\Lambda=180^{\circ}$ and $H=0^{\circ}-90^{\circ}$.

A superposition of the experimental drag curves in Fig. 2.10 for the test cases involving $\Lambda=180^{\circ}$ shows a possible relationship to the pitch angle amplitude. As the pitching amplitude decreases so do the post half-cycle peaks and the values predicted by the reduced-order models, with $H=90^{\circ}$ having the greatest value, $H=0^{\circ}$ having the lowest value, and in-between values of $H$ following suit.

This may also be connected with the shed vortices left in the wake of the wing. Dynamic stall occurs while the leading edge vortex remains attached to the upper surface of the airfoil which eventually rolls off, detaches, and gets 
left in the airfoils wake. The cyclical nature of the wings motion causes it to interact with its own wake, and thus these post-dynamic stall shed vortices. Consequently, a similar type of shed vortex interaction may also be causing the discrepencies between the dynamic stall model and experimental data observed during the half-cycle analysis. The interaction between shed vortex, airfoil, and the impact on observed forces is investigated in greater detail by Lua et al. [15].

In the end the phenomenon causing this post half-cycle peak is not accounted for by either model, as evidenced by the absence of this feature in both model produced force curves. The source of this phenomenon, for example the wing interacting with its own wake during stroke reversal as proposed above, requires more study to ascertain.

\subsubsection{Three Degrees of Freedom}

We now extend our analysis to kinematics that involve all three degrees of freedom. Our focus is now on the sets of simulations corresponding to Fig. 8 of Sane and Dickinson's work [23]. These kinematics involve keeping the sweep and pitch amplitudes constant at $\Lambda=180^{\circ}$, and $H=45^{\circ}$. The flapping amplitude $\Phi$ is set to $+25^{\circ}$ and $-25^{\circ}$. Unlike the previous simulations where the wingbeat frequency $f$ defined the frequency of all three degrees of freedom, here it defines the frequency of only sweeping and pitching. The set of simulations depicted in Fig. 2.11 form an oval pattern with the flapping frequency at $f$. The other set depicted in Fig. 2.12 form a figure-of-eight pattern with the flapping frequency at $2 f$. Through each of these 4 simulations the trapezoidal parameters were also held constant at $\Delta \tau=0.1, \tau_{0}=-0.1$, and $\tau_{f}=-0.05$. 


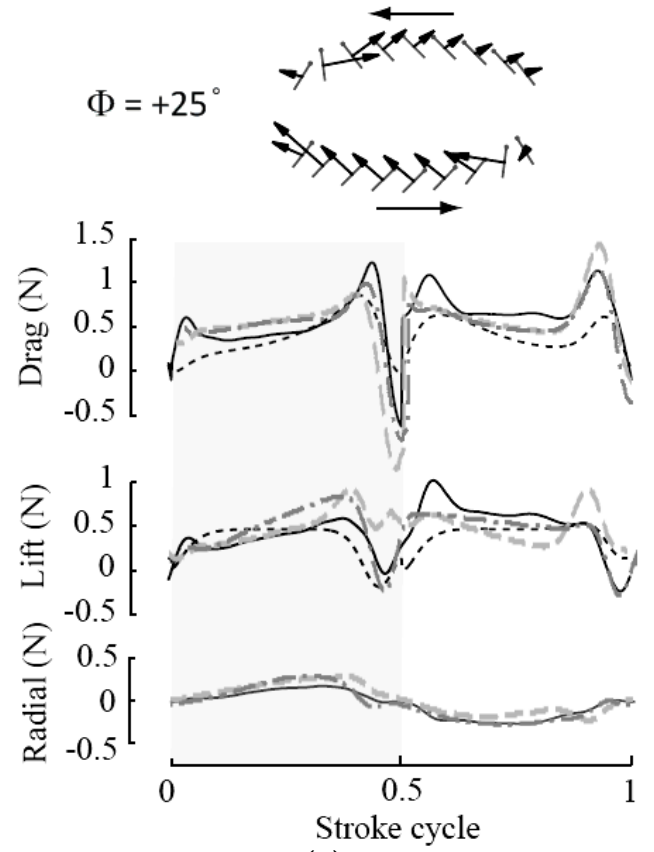

(a)
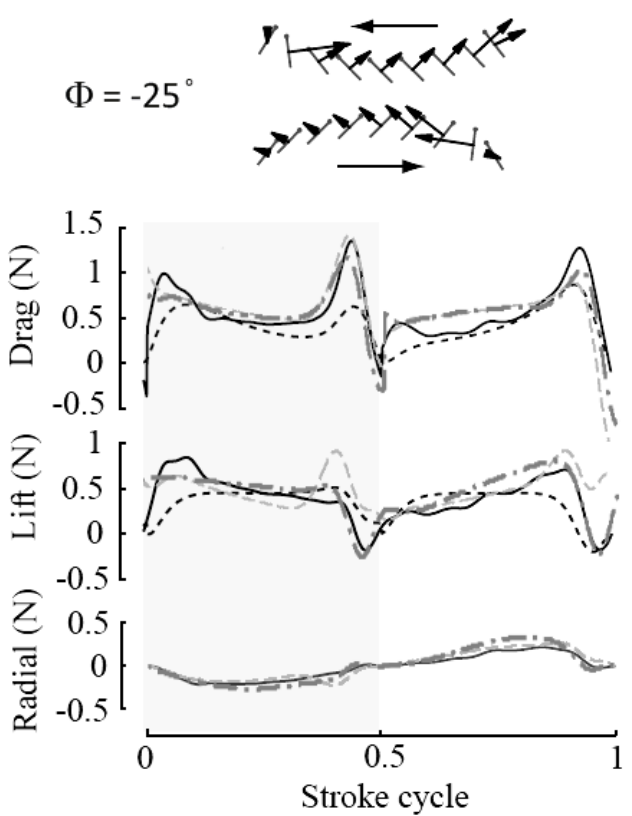

(b)

Figure 2.11: Lift and drag profiles for $\Lambda=180^{\circ}, H=45^{\circ}$, and $\Phi= \pm 25^{\circ}$. The frequency for $\phi(t)$ is set to the wingbeat frequency $f$.

As before we first focus on the lift force curves in our analysis and begin with Fig. 2.11. We can see a similar trend as with some of the simpler kinematics where differences between the reduced-order models arise during the half-cycle. The rotational lift based model seems to miss the troughs during the half-cycle, while the dynamic stall based model captures the trends but overestimates them. The mid-cycle behavior for both models is very similar and relatively faithful to the experimental data. This half-cycle and mid-cycle behavior with regards to lift is also apparent in Fig. 2.12 where the flapping frequency is set to twice the wingbeat frequency. 


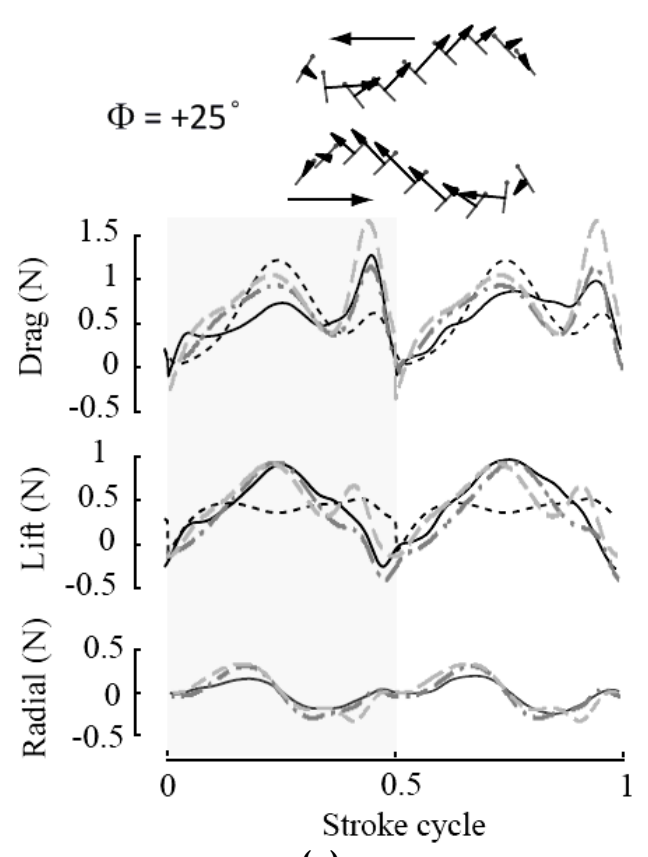

(a)

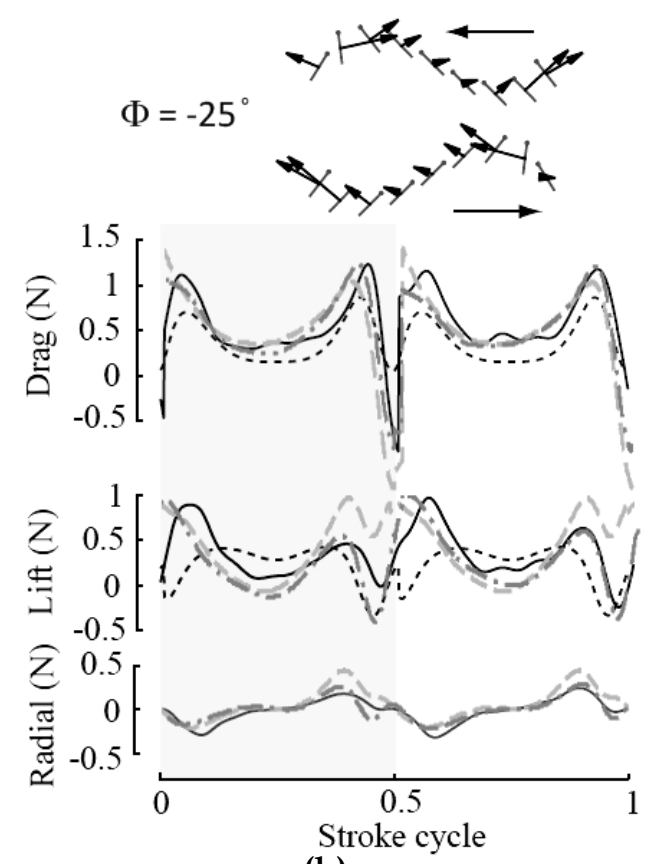

(b)

Figure 2.12: Lift and drag profiles for $\Lambda=180^{\circ}, H=45^{\circ}$, and $\Phi= \pm 25^{\circ}$. The frequency for $\phi(t)$ is set to twice the wingbeat frequency $f$.

Unlike the two degree of freedom test cases, the full three degree of freedom cases show no missing salient features in the drag curves. The only discrepancies between the experimental data and both models arises around the halfperiod, being most evident in the left plot of Fig. $2.11\left(\Phi=+25^{\circ}\right)$ and the right plot of Fig. $2.12\left(\Phi=-25^{\circ}\right)$ where the half-period troughs are being overestimated, especially by the rotational lift based model.

\subsubsection{Combining Models}

As we have seen, the dynamic stall and rotational lift concepts have differing behaviors and characteristics with respect to each other; yet both also share cer- 
tain similarities to the experimental data. This leads us to perceive each concept almost as a kind of 'mode' of flapping wing aerodynamics and explore the possibility of combining the two concepts in such a way as to gain even greater agreement with experimental data.

The circulation term is now modified to include the influence from both dynamic stall and rotational lift. Combining these approaches we now represent our circulation as

$$
\Gamma=\frac{1}{2} C_{L s} c \sqrt{v_{x^{\prime}}^{2}+v_{z^{\prime}}^{2}}+s_{d} \frac{1}{2} C_{L d} c \sqrt{v_{x^{\prime}}^{2}+v_{z^{\prime}}^{2}}-s_{r} C_{R} c^{2} \dot{\eta}
$$

where the first term again corresponds to translation, second term to dynamic stall, and the third term to rotation. This combined approach will henceforth be called the BGG model. The newly introduced coefficients $s_{d}$ and $s_{r}$ act as influence factors and determine the extent to which the corresponding phenomenon is expressed in the circulation term, with 0 being no expression and 1 being full expression. 


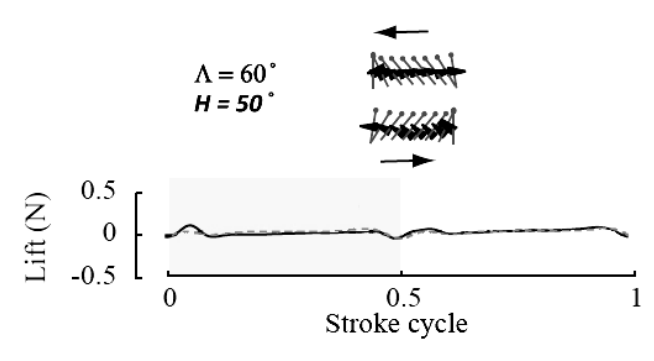

(a)

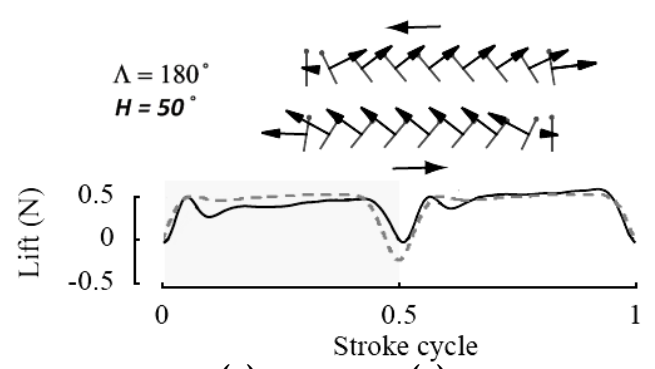

(c)

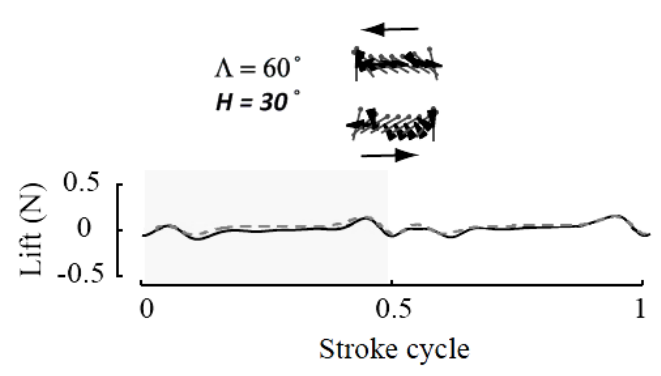

(e) (a)

(c)

(e)

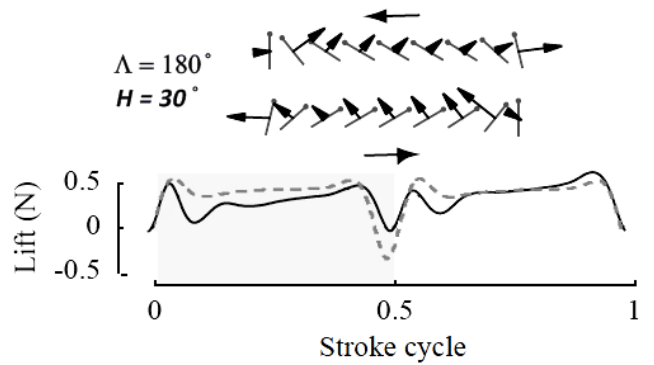

(b) (b)

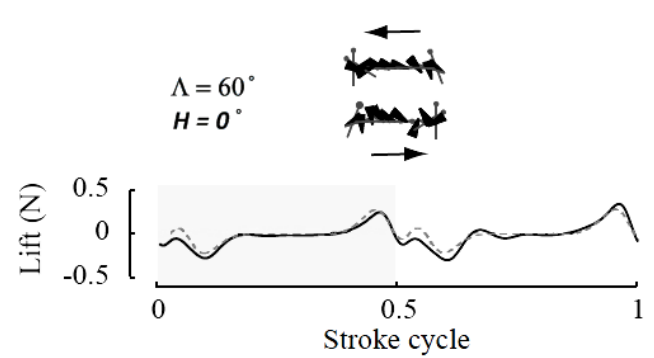

(d)

(d)

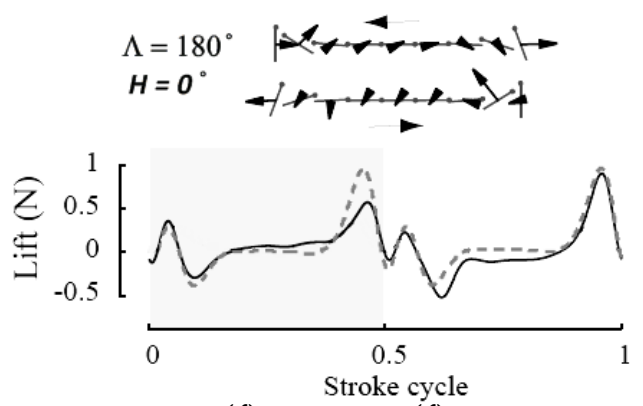

(f)

(f)

Figure 2.13: Lift curves for 6 of the 8 two degree of freedom test cases. The dark solid line is the experimental data and the lighter dashed line is the combined model with appropriate values for the influence factors $s_{d}$ and $s_{r}$.

This modified form of the circulation expression (Eq. 2.10) is used to reproduce 6 of the 8 two degree of freedom test cases. The cases involving $H=90^{\circ}$ are not reproduced due to the lift being near or at zero, thus generating uninteresting results. As can be seen in Fig. 2.13, with appropriate values for $s_{d}$ and $s_{r}$ chosen for each test case our combined model generates force curves with considerable agreement to the corresponding experimental data. The values used 
in Fig. 2.13 are: (a) $s_{d}=1$ and $s_{r}=.5$, (b) $s_{d}=.4$ and $s_{r}=0$, (c) $s_{d}=.4$ and $s_{r}=0$, (d) $s_{d}=1$ and $s_{r}=.5$, (e) $s_{d}=1$ and $s_{r}=.5$, and (f) $s_{d}=1$ and $s_{r}=.4$. These values were tuned ad hoc and are specific to the associated test cases. A study of what parameters contribute to and the type of impact they have on $s_{d}$ and $s_{r}$, as well as the form these coefficients should take (possibly a time dependent form) is left for future work.

\subsection{Conclusions}

We have built a low-order phenomenological model with which to evaluate the behavior of the rotational lift and dynamic stall based modeling concepts with respect to fluid behavior seen in flapping wings. Under the kinematics analyzed the dynamic stall and rotational lift based models each encompass certain aspects of the salient features of the lift and drag. Each approach has its own respective influencing factors that lead to differing behaviors and results in different characteristics of the experimental data being expressed in each of the approaches.

The differences in both approaches are leveraged in a combined model to generate force curves with considerably better correlation to the experimental data. The ad hoc values for the influence factors $s_{d}$ and $s_{r}$ in the present study show the potential for a combined model to adhere more faithfully to what is observed in experiments. However, a more generalized form of $s_{d}$ and $s_{r}$ based on physical parameters, possibly taking the form of a lumped parameter coefficient, is a focus of future work. The investigation of what parameters factor into these coefficients, such as Reynolds number or reduced frequency, is an area of 
continuing research.

We have seen that combining concepts offers a feasible approach to developing a low-order model with which to integrate into other numerical frameworks such as in optimization and parametric studies. On going work will include simulation work on the canonical wing maneuvers described earlier to begin to tease out the relationship between varying aspects of wing kinematics and fluid phenomena, as well as the influence dimensionless quantities such as Reynolds number, reduced frequency, and Strouhal number have on phenomenon behavior and the extent to which our current modeling methods capture these behaviors. This will help to better inform the tuning of the specific model parameters. This in conjunction with parametric studies will increase our understanding of the parametric space in which our current methods are adequate in simulating flapping wings. 
CHAPTER 3

\section{PARAMETRIC STUDY OF THE UNSTEADY AERODYNAMICS OF FLAPPING WINGS IN HOVER}

\subsection{Nomenclature}

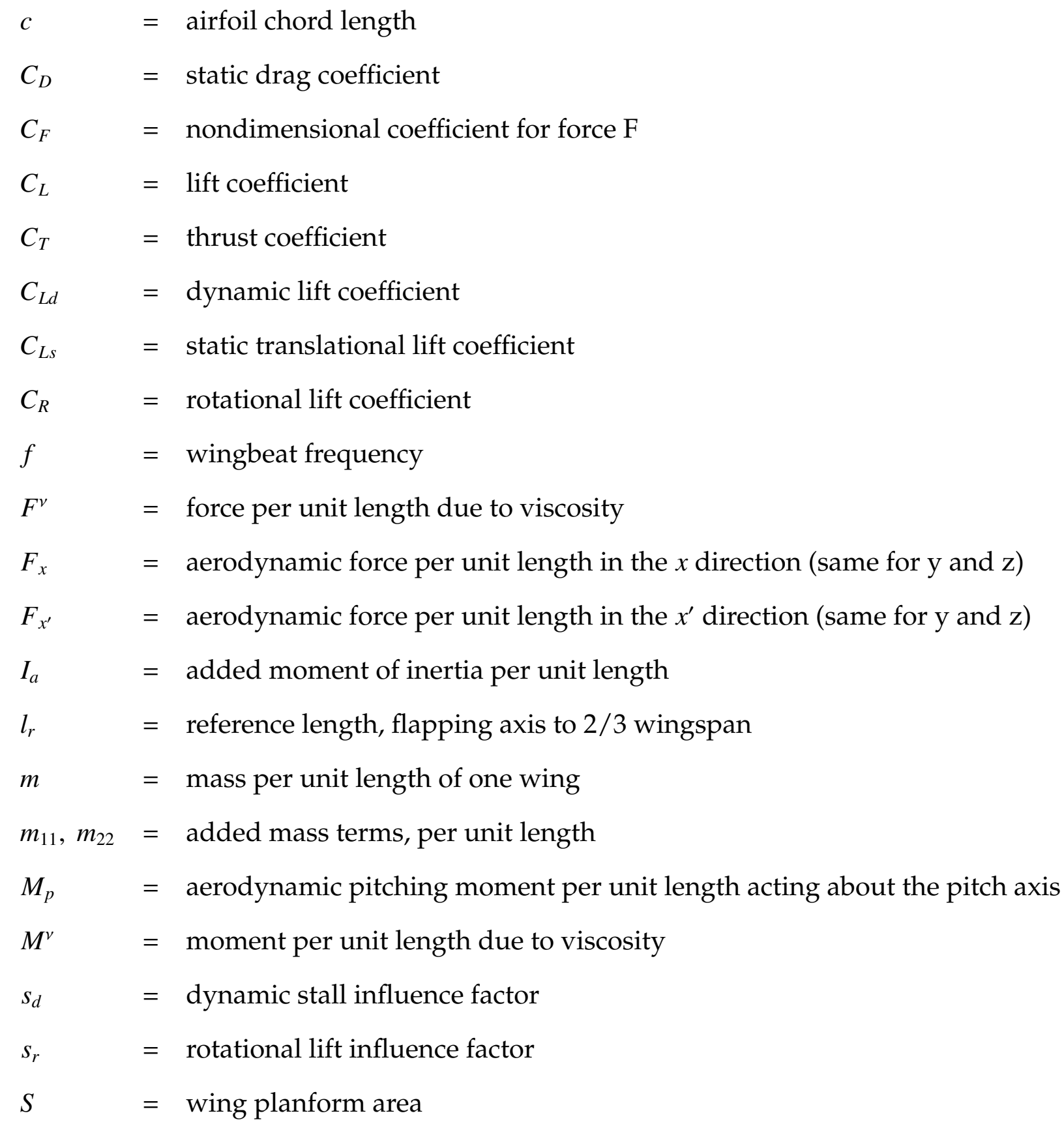




$$
\begin{array}{ll}
t & =\text { time } \\
U_{c} & =\text { characteristic velocity } \\
U_{\infty} & =\text { freestream fluid velocity } \\
v_{x} & =\text { airfoil velocity component in the } x \text { direction (same for } \mathrm{y} \text { and } \mathrm{z} \text { ) } \\
v_{x^{\prime}} & =\text { airfoil velocity component in the } x^{\prime} \text { direction (same for } \mathrm{y} \text { and } \mathrm{z} \text { ) } \\
V_{x} & =\text { body velocity component in the } x \text { direction (same for } \mathrm{y} \text { and } \mathrm{z} \text { ) } \\
V_{0} & =\text { reference velocity } \\
\alpha & =\text { angle of attack } \\
\lambda(t) & =\text { sweep angle } \\
\Lambda & =\text { sweep amplitude } \\
\phi(t) & =\text { flapping angle } \\
\Phi & =\text { flapping amplitude } \\
\eta(t) & =\text { pitching angle } \\
H & =\text { pitching amplitude } \\
\Gamma & =\text { circulation } \\
\rho_{\infty} & =\text { ambient fluid density } \\
\tau_{0} & =\text { flip start, expressed as fraction of wingbeat period } \\
\tau_{f} & =\text { flip duration midpoint, expressed as fraction of wingbeat period } \\
\tau_{v} & =\text { dimensionless vortex time } \\
\Delta \tau & =\text { flip duration, expressed as fraction of wingbeat period } \\
\omega & =
\end{array}
$$

\subsection{Introduction}

The unique attributes of unmanned aerial vehicles (UAVs) have driven their increased application in many areas. The lower cost and increased ease of pro- 
duction, as well as mitigated risk to human life make UAVs ideal for experimental aerodynamics and flight research. Morphing aircraft research such as wing shape change, re-orientation of tail and wing planform sections, and inflatable wings make use of UAVs extensively [11].

UAVs also have a central role in the study of the unsteady aerodynamics seen in flapping wings [21, 22, 28, 34]. Flapping wing flight, otherwise known as ornithopter flight, evinces a host of unsteady aerodynamic phenomena, such as dynamic stall, that are not present in classical, fixed-wing aerodynamics [28]. These phenomena are used by birds and insects to accomplish aerial feats that are either very difficult or impossible with conventional, fixed-wing aircraft and, as such, are being studied for advancing current flight capabilities.

UAVs have also seen use in a variety of different flight capacities. They are being applied to fulfill roles such as surveillance, reconnaissance, monitoring of disaster zones, and delivering medicine and supplies to remote areas. These are the types of roles that may require capabilities such as perching and hovering that exist outside of the flight regime of fixed wing aircraft, but that we see birds and insects accomplish routinely.

In addition, these disparate applications demand performance such as payload capacity, agility, and persistence in the field, that must be achieved in different ways depending on the application and situation. As a result, a need arises to perform design studies around flapping-wing UAVs to explore their capabilities and help guide the design of an ornithopter optimized for a particular application.

For the purposes of design and optimization studies, a low-computational- 
cost, numerical framework is ideal. A common approach for simulating fluid behaviour is to simulate the 3D Navier Stokes equations with commercial codes such as Fluent. However, even with appropriate simplifications, the computational cost is too high for iterative algorithms such as for the intended design and optimization studies, and as such are not often used for flapping wings.

Low-order numerical modeling, on the other hand, sacrifices the comprehensiveness and accuracy of CFD simulations for simplicity and efficiency. Low-order models take a phenomenological approach and focus on only capturing the more important, governing aspects of a given problem which leads to much lower computational costs when compared with CFD simulations. With respect to unsteady aerodynamics, these models quantify phenomena such as added mass, circulatory effects, and viscous effects that we see from physical experimentation by applying established theory and developing semi-empirical terms based on available experimental data $[6,34,35]$.

The impetus for the present study is to demonstrate the potential of loworder models to characterize a large parameter space. These attributes are ideal for integration into the types of iterative and multi-tiered numerical frameworks seen in such investigations. These types of efforts will help enable future work in UAV design and optimization for specific roles and will help explore the capabilities of UAV ornithopters.

In this study we explore the parametric space of an ornithopter in a hovering flight configuration. The low-order phenomenological model developed by Gomez, Bryant, and Garcia is used in a greater investigative framework to generate the parametric study by varying certain kinematic variables [10]. The experimental data of Sane and Dickinson, in the range of $10^{2}<\operatorname{Re}<10^{3}$ and a 
flapping frequency of $0.17 \mathrm{~Hz}$, is used to guide the choice of parametric space to investigate and to help guide our analysis by forming a basis of comparison with the generated numerical data [23]. With this framework, we first study how the combined model compares to the experimental data of interest and analyze where differences may arise. We then move on to generating parametric studies to analyze how the stroke-averaged aerodynamic forces change over the space. This is also used to gain a more general understanding of the characteristics of the combined model, as well as its capacity as a simplified model utilizing limited finite data to model a more complex system.

\subsection{Modeling Approach}

\subsubsection{Wing Kinematics}

The seminal work done by Dudley and Ellington into flapping wing mechanics is used often in ornithopter studies, and is used here as the basis for defining our wing kinematics [8]. In particular, we define our wing kinematics the same as that in Gomez, Bryant, and Garcia [10]. A brief synopsis is given here.

Wing motion is described by three degrees of freedom: the flapping angle $\phi(t)$, pitch angle $\eta(t)$, and sweep angle $\lambda(t)$. As can be seen in Fig. 3.1 these angles define the relationship between the three-dimensional vector orientation of a body-centered (unprimed) coordinate system and a series of coinciding (primed) coordinate systems corotating with each 2D airfoil section that comprises the wing. Simple gimbal rotation matrices are used, each one rotating about a primary axis and associated with a defined degree of freedom. These 
are shown in Eq. 3.1.

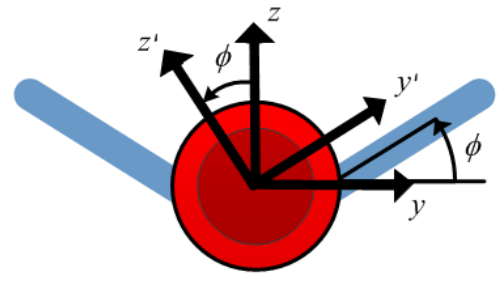

(a) Front view

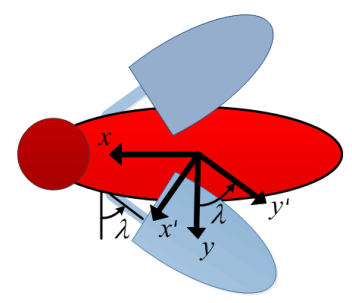

(b) Top view

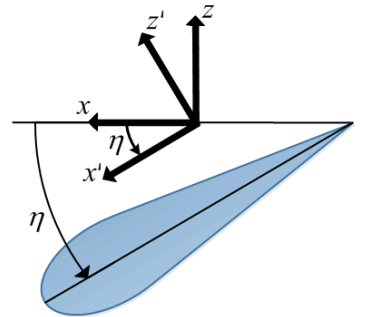

(c) Airfoil-centered view

Figure 3.1: Coordinate axes are shown in various views. Arrows point in the positive direction.

$$
R_{\phi}(t)=\left[\begin{array}{ccc}
1 & 0 & 0 \\
0 & \cos (\phi) & \sin (\phi) \\
0 & -\sin (\phi) & \cos (\phi)
\end{array}\right], \quad R_{\lambda}(t)=\left[\begin{array}{ccc}
\cos (\lambda) & \sin (\lambda) & 0 \\
-\sin (\lambda) & \cos (\lambda) & 0 \\
0 & 0 & 1
\end{array}\right], \quad R_{\eta}(t)=\left[\begin{array}{ccc}
\cos (\eta) & 0 & -\sin (\eta) \\
0 & 1 & 0 \\
\sin (\eta) & 0 & \cos (\eta)
\end{array}\right]
$$

We have studied the three waveforms shown in Fig. 3.2: the sine, triangle, and trapezoidal waveforms for the heaving, sweep, and pitch, respectively, which mimic the flight of birds and insects. When combined, these waveforms can produce patterns that roughly approximate the wing patterns of various insects including the fruit fly $[8,33]$. As in Dickinson and Sane's work, the trapezoidal waveform includes a flip duration $\Delta \tau$, the time it takes for the wing to flip, and a flip start $\tau_{0}$, the point in time that the flip begins, with both expressed as a fraction of wingbeat period [23].

A more in-depth treatment of the wing kinematics can be found in the previous work [10]. 

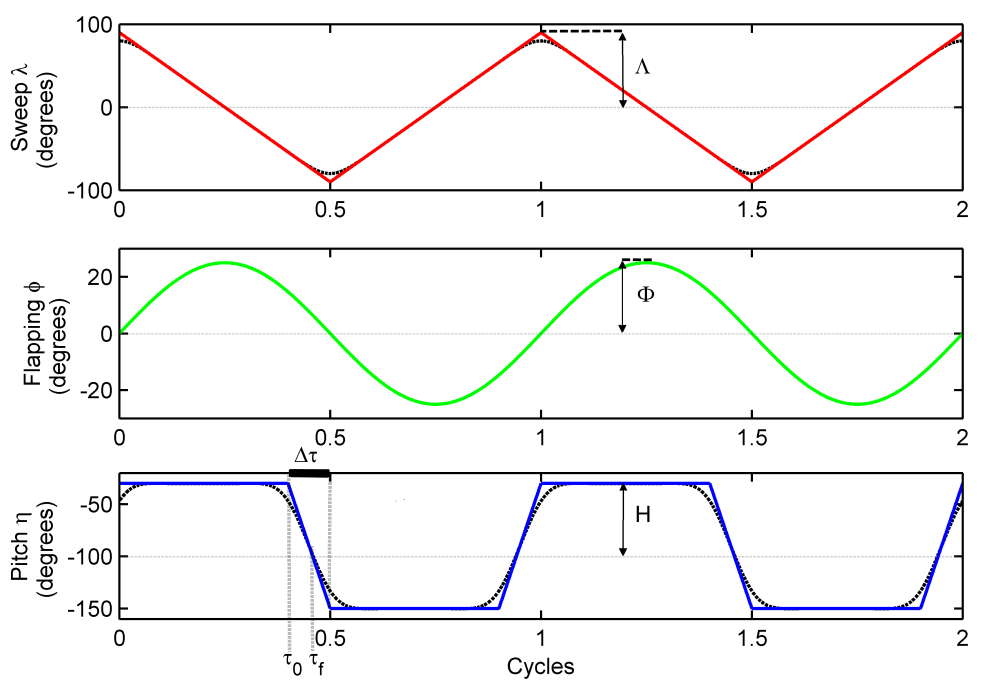

Figure 3.2: The three waveforms chosen for the wing kinematics. Solid lines are the base waveforms. Rounded lines are the smoothed waveforms.

\subsubsection{Aerodynamic Modeling}

We closely follow the model developed by Gomez, Bryant, and Garcia [10]. In this model, the circulation is written as

$$
\Gamma=\frac{1}{2} C_{L s} c \sqrt{v_{x^{\prime}}^{2}+v_{z^{\prime}}^{2}}+\frac{1}{2} C_{L d} c \sqrt{v_{x^{\prime}}^{2}+v_{z^{\prime}}^{2}}-C_{R} c^{2} \dot{\eta}
$$

where the first term corresponds to translation from classical aerodynamic theory, the second corresponds to dynamic stall, and the third corresponds to rotational lift.

The rotational lift term we use here comes from Anderson, Pesavento, and Wang's rotational-lift-based aerodynamic model [1]. This quasi-steady approach assumes the aerodynamic forces generated by an airfoil are due to its simultaneous heaving (translating) and pitching (rotating) motions in two dimensions. Here we assume the aerodynamic forces arise from two seperate 
sources: translational (circulatory) lift $C_{L s}$ generated by heaving, and rotational lift $C_{R}$ generated by pitching.

The dynamic stall term comes from the model by Bryant, Gomez, and Garcia [4]. Dynamic stall arises from leading-edge vortices (LEVs) that temporarily increase lift properties while attached to the upper wing surface. This liftenhancing phenomenon was first observed experimentally in bird and insect flight $[33,9,30,31]$.

With the circulation defined in Eq. 3.2, the aerodynamic forces per unit span generated by a moving airfoil as expressed by this combined model are

$$
\begin{gathered}
F_{x^{\prime}}=-\left(m+m_{22}\right) \dot{\eta} v_{z^{\prime}}-\rho_{\infty} \Gamma v_{z^{\prime}}-m_{11} \dot{v_{x^{\prime}}}-F_{x^{\prime}}^{v} \\
F_{z^{\prime}}=\left(m+m_{11}\right) \dot{\eta} v_{x^{\prime}}+\rho_{\infty} \Gamma v_{x^{\prime}}-m_{22} \dot{v_{z^{\prime}}}-F_{z^{\prime}}^{v}
\end{gathered}
$$

where the four terms, in turn, account for (1) the effects of calculating forces from a rotating reference frame, (2) circulation effects, (3) added mass effects, and (4) viscous effects. These forces are converted into lift and thrust coefficients by following the nondimensionalizing approach of Nagai et. al. [18]:

$$
\begin{gathered}
C_{F}=F /\left(0.5 \rho_{\infty} V_{0}^{2} S\right) \\
V_{0}=2 \pi f \Lambda l_{r}
\end{gathered}
$$

All other terms and expressions are the same as in Bryant, Gomez, and Gar- 
cia's previous works, discussed in detail in [10,4].

\subsection{Results and Discussion}

\subsubsection{Verification of Numerical Model}

We first compare the combined model to the experimental data being used as the basis for our parametric study. The experimental data used is that of Sane and Dickinson's study of ornithopters in the hovering configuration [23]. For the hovering case, we are particularly interested in the lift averaged over one flapping cycle, here called the stroke-averaged lift. The numerical and experimental data are shown in Fig. 3.3(a) \& 3.3(b), where $\Lambda$ is kept constant at $90^{\circ}$ for Fig. 3.3(a) and at $30^{\circ}$ for Fig. 3.3(b). The circular markers indicate experimental data points, the square markers the numerical data points from the combined model, and the diamond and triangle markers the numerical data points from the isolated dynamic stall and rotational lift contributions, respectively.

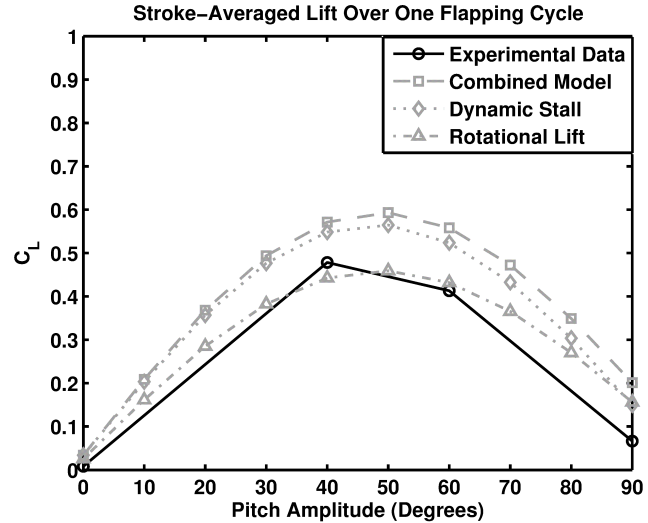

(a) Stroke Amplitude $\Lambda=90^{\circ}$

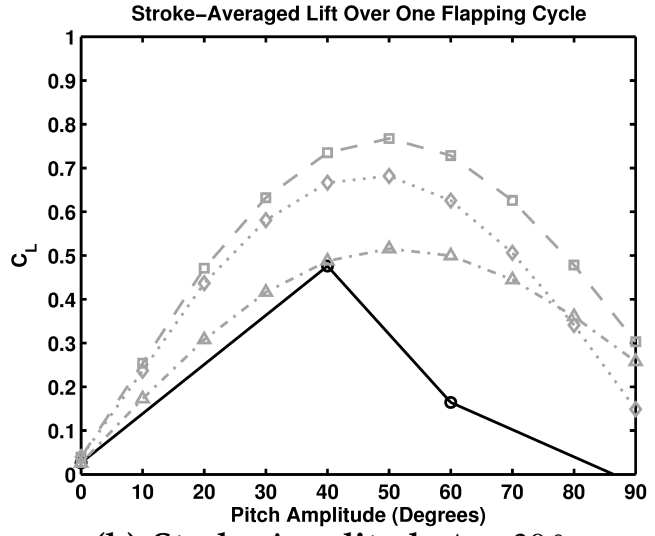

(b) Stroke Amplitude $\Lambda=30^{\circ}$

Figure 3.3: Stroke-averaged lift versus pitch amplitude. 
Our goal is to gain an understanding of how well the low-order combined model captures the general behavior of the lift force with respect to the experimental data. This is done by observing where the model performs well and where discrepencies arise. Through analysis of both the experiments and numerical model we can gain insight into the mechanisms that bring about these behaviors and gain a broader picture of the combined models capacity.

For the case of $\Lambda=90^{\circ}$, the combined model shows good correlation with the experimental data. In Fig. 3.3(a) we see the combined model capturing the general behavior of the experimentally derived stroke-averaged lift, with lift increasing and peaking as we approach $40^{\circ}<H<60^{\circ}$. In this figure the lift from the combined model peaks at $H=50^{\circ}$ and the experimental data peaks at $H=40^{\circ}$, keeping in mind that we have a limited number of experimental data points with which to compare and thus do not have experimental information for $40^{\circ}<H<60^{\circ}$.

For the case of $\Lambda=30^{\circ}$, greater discrepencies arise for $H>40^{\circ}$. In Fig. 3.3(b) we see the combined model has the same general behavior as in Fig. 3.3(a), with the experimental data on the other hand showing the same behavior only up to $H=40^{\circ}$. For $H=60^{\circ}$ the experimental lift falls considerably and goes slightly negative for $H=90^{\circ}$, with this behavior not being reflected in the combined model.

Several factors can be contributing to these discrepencies in behavior. In addition to the total lift we also see the contributions from the dynamic stall and rotational lift terms of the combined model in Fig. 3.3(b). We see again that dynamic stall and rotational lift exhibit the same behavior that they did for $\Lambda=90^{\circ}$, even though the experimental data does not. This indicates a possible 
limitation of the combined model for small stroke amplitudes, in this case $\Lambda=$ $30^{\circ}$. This is a potential consequence of the low-order nature of the model: using simplified schemes and limited 2D data to model a more complex system. For example, at the heart of the dynamic stall term is a second-order differential equation that models the forces from fully formed shed vortices. Because of this the model may be missing more complex interactions such as underdeveloped vortices and secondary and tertiary wing-vortex interactions that may arise for lower stroke amplitudes. 


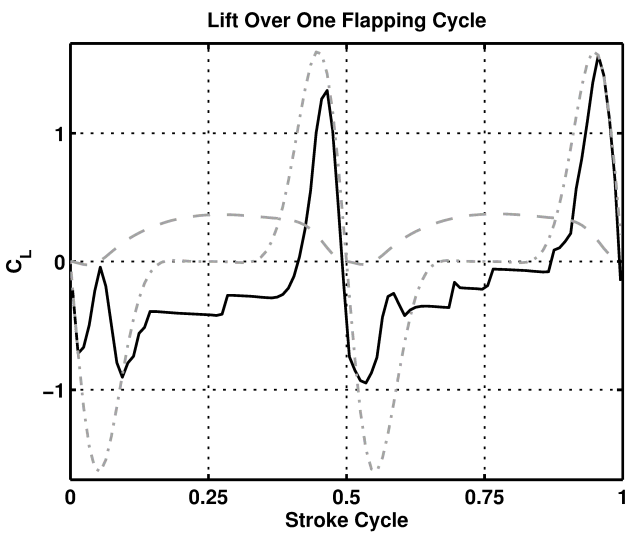

(a) $\Lambda=30^{\circ}, H=40^{\circ}$

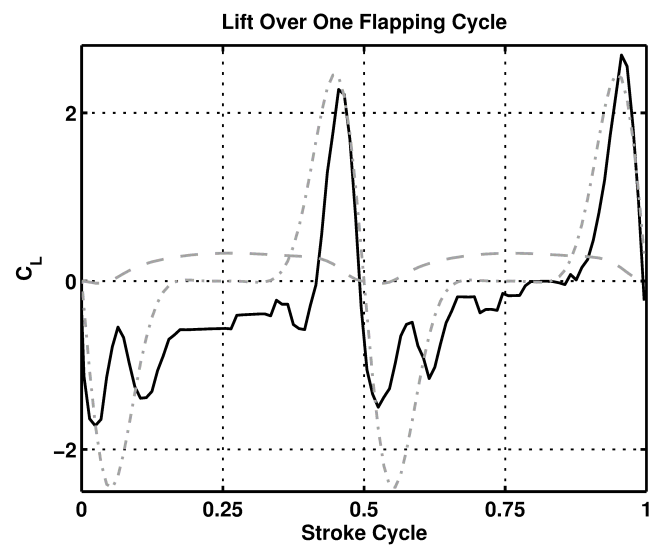

(c) $\Lambda=30^{\circ}, H=60^{\circ}$

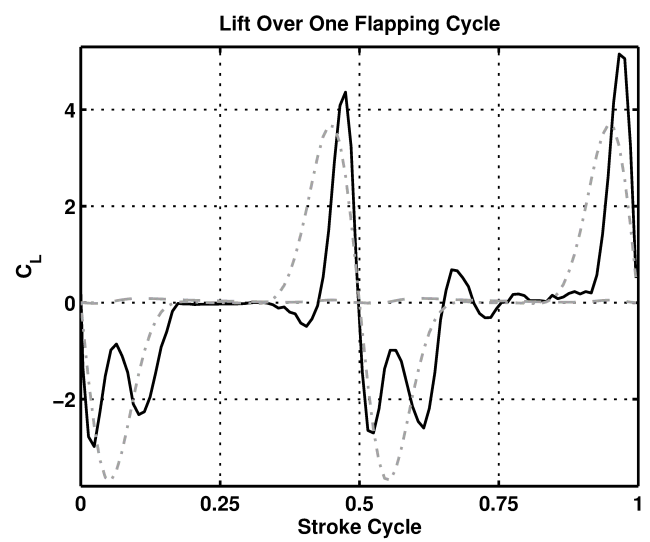

(e) $\Lambda=30^{\circ}, H=90^{\circ}$

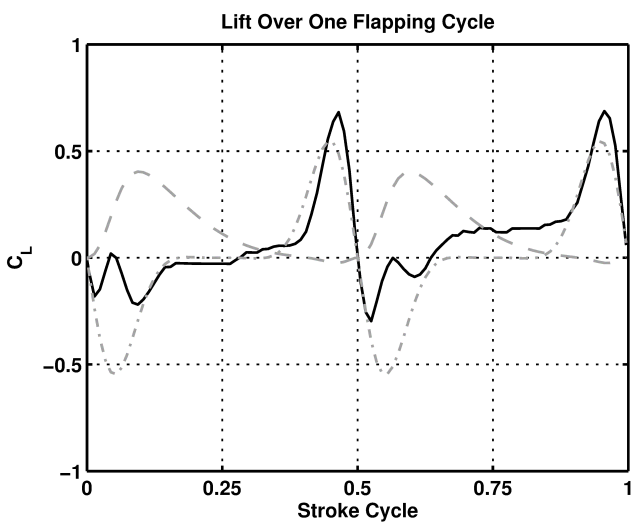

(b) $\Lambda=90^{\circ}, H=40^{\circ}$

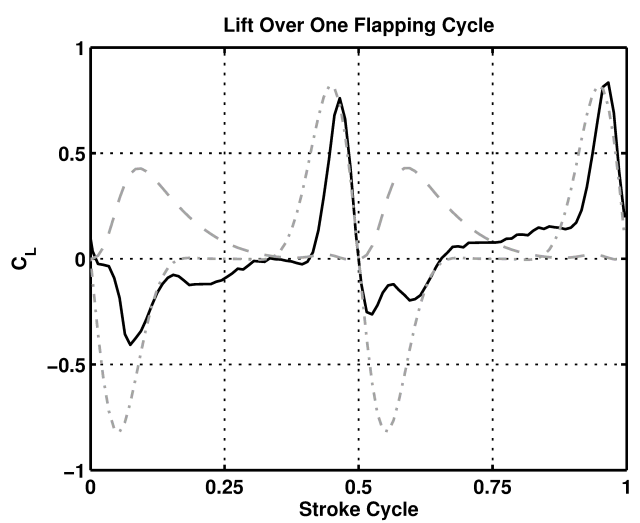

(d) $\Lambda=90^{\circ}, H=60^{\circ}$

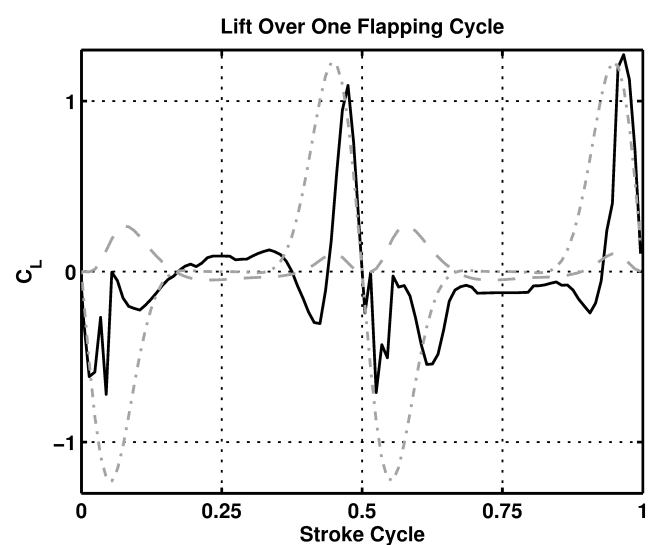

(f) $\Lambda=90^{\circ}, H=90^{\circ}$

Figure 3.4: Experimental data minus forces not associated with Dynamic Stall and Rotational Lift.

To better understand the roles that dynamic stall and rotational lift play in 
these discrepencies it is helpful to compare these components of the combined model directly with the corresponding quantities in the experimental data. Despite not having such a breakdown of the experimental data, we can proceed by making an assumption about the modeling of the forces not associated with dynamic stall and rotational lift utilized in our combined model. We know that these terms, especially when compared to dynamic stall and rotational lift, are much better understood and thus can assume with reasonable certainty that they are being accurately modeled. This assumption allows us to subtract these numerical quantities directly from the experimental data, leaving us with a reasonable approximation of the quantities in the experimental data directly associated with dynamic stall and rotational lift.

This direct comparison of dynamic stall and rotational lift components is plotted in Fig. 3.4. For each sets of figures the black solid line represents the isolated experimental data, the grey dashed line represents the isolated numerical dynamic stall component and the grey dot-dashed line represents the isolated numerical rotational lift component. The figures associated with $H=0^{\circ}$ are not shown due to the force curves being zero and thus not showing any meaningful insight.

We can see that for the cases where $\Lambda=90^{\circ}$ dynamic stall and rotational lift are working together to approximate the salient features of the experimental data. For the cases where $\Lambda=90^{\circ}$ we see rotational lift solely covering the important salient features and dynamic stall not meaningfully contributing to a better approximation of the experimental data. This difference, and the ineffectiveness of the dynamic stall term at the lower stroke angle, points toward a host of kinematic configurations where rotational lift begins to dominate and the dy- 
namic stall term in its current form struggles to compensate. This represents an extreme where the dynamic stall ODE of our combined model reaches a limit in its current form and may require more invetigation for greater refinement.

Additional insight can be gained by studying the original experimental data. Looking back at Sane and Dickinson's original work, specifically the force versus time plots, we can see for the cases with $\Lambda=30^{\circ}$ the lift is at or hovering very close to zero, with few very prominent features visible [23]. This low lift production indicates that, atleast from an engineering design stand point, smaller stroke angles such as $\Lambda=30^{\circ}$ is of less interest as this is not ideal for hovering flight.

We have now gained an understanding of the capacity of the combined model to capture the general behaviors seen in the experimental data. Through analysis of both the experiments and numerical model we have seen that for $\Lambda=90^{\circ}$, an area of interest with respect to engineering design due to higher lift prodcution, there is strong correlation between experimental and numerical data. For lower stroke amplitude values discrepencies arise which may be an indication of the limitation of the low-order model and our dynamic stall term. However these areas, as represented by the $\Lambda=30^{\circ}$ experimental data, are of less interest due to little to no lift production.

\subsubsection{Observations of Physical Mechanisms}

The combined model is used to characterize the parametric space encompassed by the experimental data of Sane and Dickinson used in our verification. The stroke-averaged lift changing over the space is shown in Fig. 3.5, where we vary 
both the sweep and pitch amplitudes $0^{\circ}-90^{\circ}$.

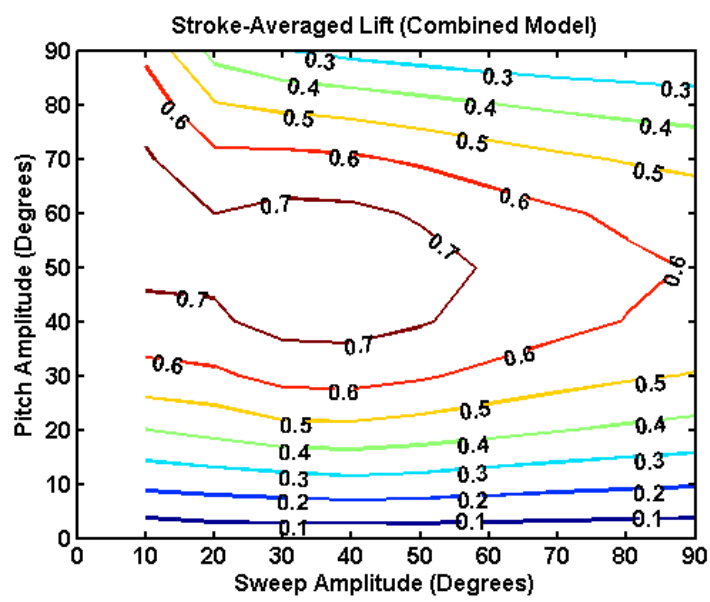

Figure 3.5: Stroke-averaged lift for $\Lambda=0^{\circ}-90^{\circ}$ and $H=0^{\circ}-90^{\circ}$.

As also seen in Fig. 3.3(a) \& 3.3(b), the contour plot of Fig. 3.5 shows the stroke averaged lift peaks around a pitch amplitude of $50^{\circ}$ for all values of the stroke (sweep) amplitude, with lift having the highest magnitude in the parametric area around $20^{\circ}<\Lambda<50^{\circ}$ and $40^{\circ}<\Lambda<60^{\circ}$, and a maximum value around a stroke amplitude of $45^{\circ}$.

There also exists areas of zero or near-zero lift in the extremes of the parametric space. The areas of zero lift occur when the pitch amplitude is $0^{\circ}$ and when the sweep amplitude is $0^{\circ}$. These results at the limits of the parametric space are consistant with hovering kinematics with a non-cambered airfoil: no lift if no stroke, and no lift if no pitch.

It should be noted that the zero lift region around $\Lambda=0^{\circ}$ is difficult to discern in Fig. 3.5. If we look at the lift force without nondimensionalization we would see that it is going to zero as stroke amplitude goes to zero. An issue arises in our nondimensionalizing term being dependent on the stroke amplitude and also going to zero as $\Lambda$ approaches zero, obfuscating this behavior. 
This illustrates one of the difficulties in analyzing hovering flight, namely the choice of an appropriate non-dimensionalizing term. This issue only arises in the limiting case of the stroke amplitude approaching zero, an area of the parametric space that from an engineering design standpoint is not of interest due to little to no lift production.

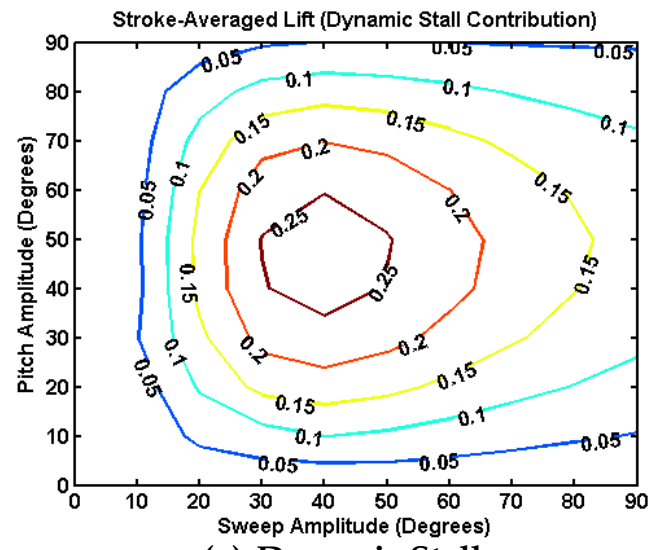

(a) Dynamic Stall

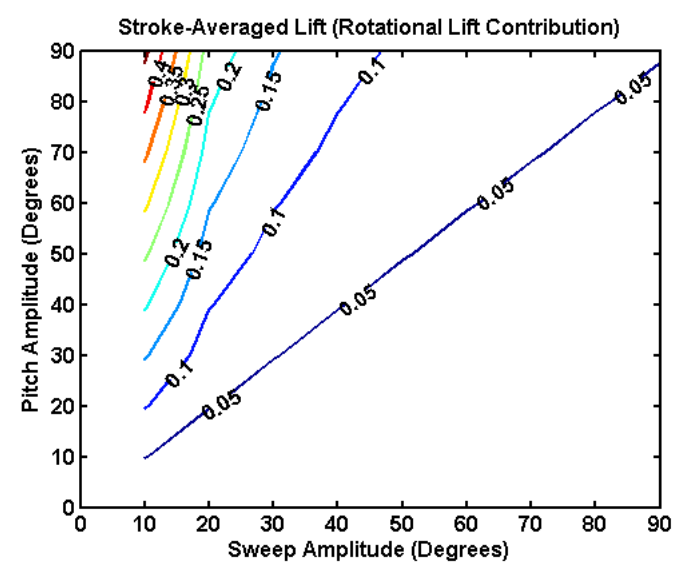

(b) Rotational Lift

Figure 3.6: Stroke-averaged lift for $0^{\circ}<\Lambda<90^{\circ}$ and $0^{\circ}<H<90^{\circ}$.

To better understand some of the observed behaviors we look at the contributions to lift from the dynamic stall and rotational lift phenomena, shown in Fig. 3.6. In Fig. 3.6(b) we can see that the contribution from rotational lift increases as pitch amplitude increases, a direct consequence of the rotational lift term being directly proportional to the pitch rate. This increasing rotational lift is also true for decreasing stroke amplitude, as decreasing stroke amplitude also leads to an increase in pitch rate. These two behaviors lead to the rotational lift contribution peaking as $H$ approaches $90^{\circ}$ and $\Lambda$ approaches $0^{\circ}$, also contributing to the spike in total lift in the upper left corner of Fig. 3.5.

Dynamic stall on the other hand shows a different behavior in the parametric space. In Fig. 3.6(a) we see the dynamic stall derived lift increasing as we 
approach the middle of the parametric space and peaking at a pitch amplitude of $50^{\circ}$ and stroke amplitude of $45^{\circ}$. This coincides very closely with the peak total lift depicted in Fig. 3.5, indicating that the main contributing factor to the behavior seen in the total lift, specifically the increasing and then peaking as we approach $H=50^{\circ}$ and $\Lambda=45^{\circ}$, is due to dynamic stall.

\subsubsection{Frequency Effects}

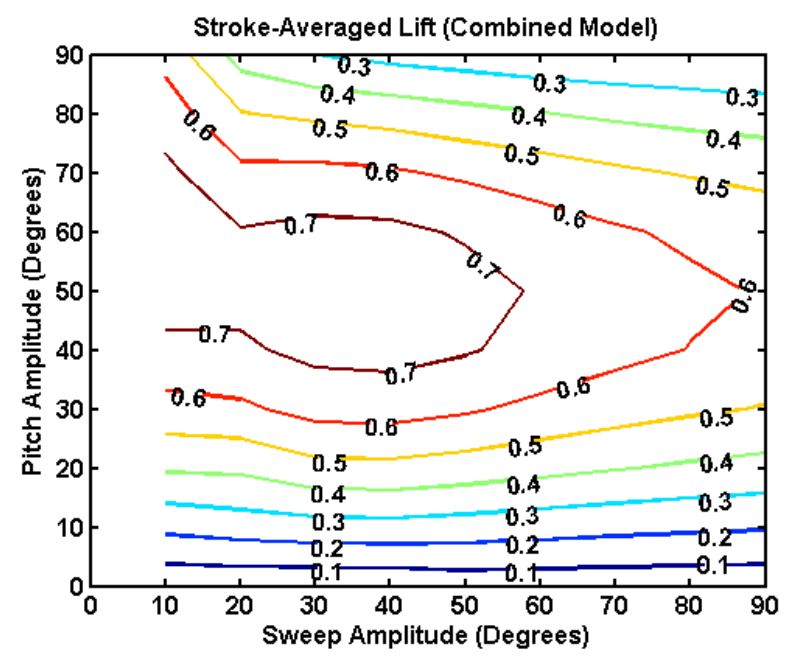

Figure 3.7: Stroke-averaged lift for $f=0.1-1 \mathrm{~Hz}$.

We now expand our parametric study by varying the flapping frequency $f$ from 0.1 to $1 \mathrm{~Hz}$. When we do this we see that the characteristic behavior, including the magnitude, of the stroke-averaged lift over the parametric space remains the same, depicted in Fig. 3.7, indicating that the combined model has frequency independence. The contributions from dynamic stall and rotational lift are also the same as for $f=0.17 \mathrm{~Hz}$.

The consistancy in magnitude across flapping frequencies is comparable to that seen in fixed-wing airfoil wind tunnel studies. In these studies the lift co- 
efficient $C_{L}$ contains a nondimensionalizing term that is dependent on and thus scales with wind speed. This in turn causes $C_{L}$, atleast at higher Reynolds numbers, to be independent of wind speed and only a function of angle of attack. This parrallels nicely with what we see in the current study; our nondimensionalizing coefficient is dependent on flapping frequency, which in turn causes our $C_{L}$ to be frequency independent and only a function of the kinematic variables $H$ and $\Lambda$.

The dynamic stall and rotational lift phenomena's dependence on behavior is not clearly undestood and requires more research to understand. The similarities described above could be an example of how to gain an understanding of complex systems by observing parrallels with simpler systems. It could, however, also potentially be due to the reduced-order nature of our approach, and as such represent a limitation of our model. Further experimental data is needed to gain insight into how well this represents physical behavior and where nonlinearities may breakdown our approach and introduce discrepencies.

\subsection{Conclusions}

We have implemented a low-order phenomenological model to explore the parametric space of an ornithopter in hovering conditions. Experimental data was used as the basis for the initial parametric space where pitch and sweep were varied to characterize how the stroke-averaged lift changes with respect to these variables. The dynamic stall phenomenon was seen to have a more significant contribution to the forces produced where the total lift increases and then peaks as we approach $H=50^{\circ}$ and $\Lambda=45^{\circ}$. 
Expanding our parametric space to explore varying flapping frequencies reveals similar trends. As we vary $f=0.1-1 \mathrm{~Hz}$ we see that the behavior and magnitude of the stroke-averaged lift over the parametric space remains the same as for $f=0.17 \mathrm{~Hz}$, indicating that the combined model has frequency independence. This also applies to the contributions from dynamic stall and rotational lift.

This study is the beginning of exploring the feasibility of using the BGG low-order model in numerical frameworks such as optimization and parametric studies. On going work will include simulation work on ornithopter forward flight configurations and exploring lift and thrust behavior over kinematic parameter spaces of interest. This will help round out our initial study of ornithopter flight from an overall stroke-averaged force generation stand point, adding to our continuously growing undertanding of flapping wing flight. 
CHAPTER 4

\section{PARAMETRIC STUDY OF THE UNSTEADY AERODYNAMICS OF FLAPPING WINGS IN FORWARD FLIGHT}

\subsection{Nomenclature}

$$
\begin{aligned}
& c=\text { airfoil chord length } \\
& C_{D} \quad=\quad \text { static drag coefficient } \\
& C_{L} \quad=\text { lift coefficient } \\
& C_{L d} \quad=\text { dynamic lift coefficient } \\
& C_{L s}=\text { static translational lift coefficient } \\
& C_{R} \quad=\text { rotational lift coefficient } \\
& f \quad=\text { wingbeat frequency } \\
& F^{v} \quad=\text { force per unit length due to viscosity } \\
& F_{x} \quad=\text { aerodynamic force per unit length in the } x \text { direction (same for } \mathrm{y} \text { and } \mathrm{z} \text { ) } \\
& F_{x^{\prime}} \quad=\text { aerodynamic force per unit length in the } x^{\prime} \text { direction (same for } \mathrm{y} \text { and } \mathrm{z} \text { ) } \\
& I_{a} \quad=\text { added moment of inertia per unit length } \\
& m \quad=\quad \text { mass per unit length of one wing } \\
& m_{11}, m_{22}=\text { added mass terms, per unit length } \\
& M_{p} \quad=\text { aerodynamic pitching moment per unit length acting about the pitch axis } \\
& M^{v} \quad=\text { moment per unit length due to viscosity } \\
& s_{d} \quad=\text { dynamic stall influence factor } \\
& s_{r} \quad=\text { rotational lift influence factor } \\
& t \quad=\text { time } \\
& U_{c} \quad=\text { characteristic velocity } \\
& U_{\infty} \quad=\text { freestream fluid velocity } \\
& v_{x} \quad=\text { airfoil velocity component in the } x \text { direction (same for } \mathrm{y} \text { and } \mathrm{z} \text { ) }
\end{aligned}
$$




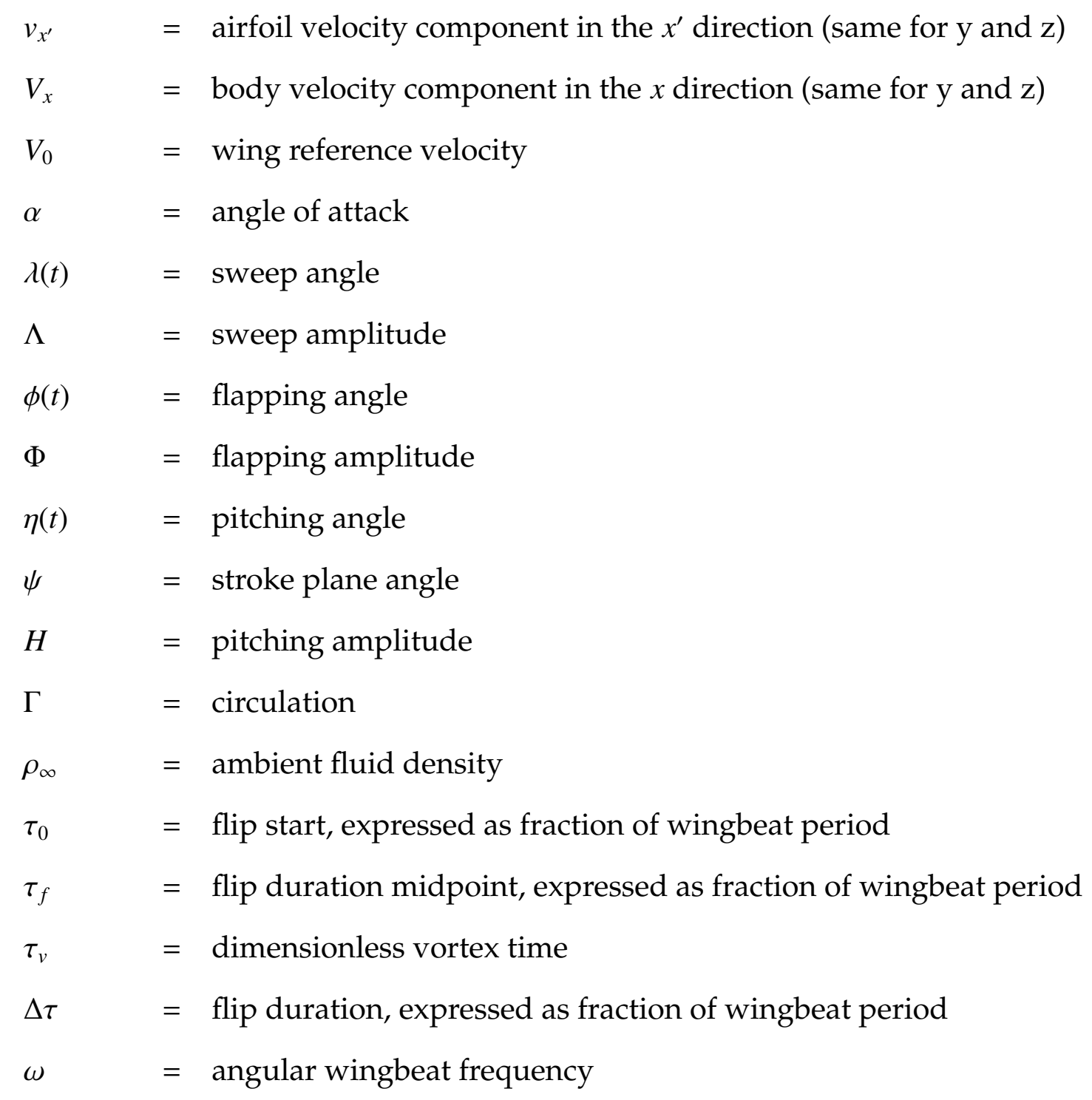

\subsection{Introduction}

Unmanned aerial vehicles (UAVs) have seen an increase of use in the modern world. These uses range from point to point delivery service to recreational video and photography. The lower cost and increased ease of production are some of the characteristics that make UAVs ideal for a wide range of applications. 
Another reason for the increased interest in $\mathrm{UAVs}$ is due to their potential flight capabilities. Ornithopter flight presents a host of unsteady aerodynamic phenomena, such as dynamic stall, that are not present in classical fixed wing aerodynamics $[20,7,19]$. These phenomena are used by birds and insects to accomplish aerial feats that are either very difficult or not possible with conventional fixed wing aircraft. As such the application of these flight capabilities to UAVs is of great interest not only from a commercial perspective but also from a scientific one [28].

UAVs have also begun to fill a number of different roles for important and sometimes critical situations. Applications such as surveillance, reconnaissance, monitoring of disaster zones, and delivering medicine and supplies to remote areas. Applications that may require capabilities such as perching and hovering that exist outside of the flight regime of fixed wing aircraft, but that we see birds and insects accomplish routinely.

For application in these roles ornithopter behavior in a forward flight configuration must be studied. Specifically the phenomena not seen in classical aerodynamics must be investigated to see the impact on flight attributes such as maneuverability and flight efficiency, and how their behavior changes with changing conditions. As a result a need arises for the ability to perform design studies around flapping wing UAVs to explore their capabilities and help guide the design of an ornithopter optimized for a particular application.

For the purposes of design and optimization studies a low-computationalcost numerical framework is ideal. Low-order numerical modeling sacrifices the comprehensiveness and complexity of CFD simulations for simplicity and efficiency. Low-order models take a phenomenological approach and focus on 
only capturing the more important, governing aspects of a given problem which leads to much lower computational costs when compared with CFD simulations. With respect to unsteady aerodynamics these models quantify phenomena such as added mass, circulatory effects, and viscous effects that we see from physical experimentation by applying established theory and developing semiempirical terms based on available experimental data $[6,34,35]$.

The impetus for the present study is to begin the ground work for investigating the forward flight behavior of ornithopters. In order for design studies on subjects such as flight trajectory and path planning to be done, an understanding of the aerodynamic forces integral to flight must first be established. This involves studying the lift and drag generated by flapping wings and analyzing how they change over a parametric space, as well as how unsteady phenomena affects there behavior.

In this study we explore the parametric space of an ornithopter in a forward flight configuration. The low-order phenomenological model developed by Gomez, Bryant, and Garcia is used in a greater investigative framework to generate the parametric study by varying certain kinematic variables [10]. The experimental data of Nagai and Hayase is used as the basis for the parametric spaces of interest [18]. With this framework we first compare the phenomenological model to the experimental data and move on to expanding the parametric space to analyze how the aerodynamic forces change. 


\subsection{Modeling Approach}

\subsubsection{Wing Kinematics}

The seminal work done by Dudley and Ellington into flapping wing mechanics is used often in ornithopter studies, and is used here as the basis for defining our wing kinematics [8]. In particular we describe wing motion by three degrees of freedom: the flapping angle $\phi(t)$, pitch angle $\eta(t)$, and sweep angle $\lambda(t)$. As can be seen in Fig. 4.1 these angles define the relationship between the three-dimensional vector orientation of a body-centered (unprimed) coordinate system and a series of coinciding coordinate systems (primed) corotating with each 2D airfoil section that comprises the wing. Simple gimbal rotation matrices are used where each one is about a primary axis and associated with a defined degree of freedom. These are expressed in Eq. 4.1.

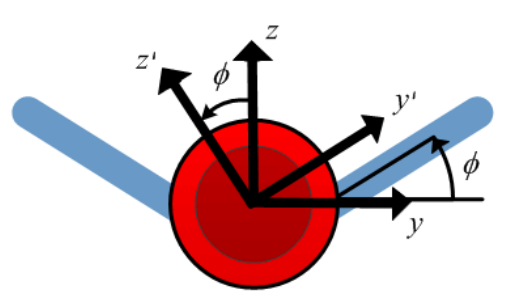

(a) Front view

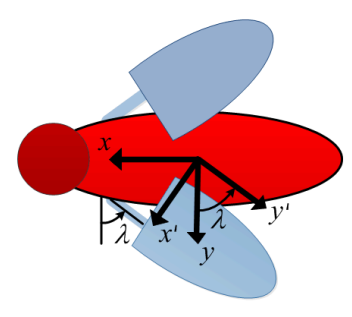

(b) Top view

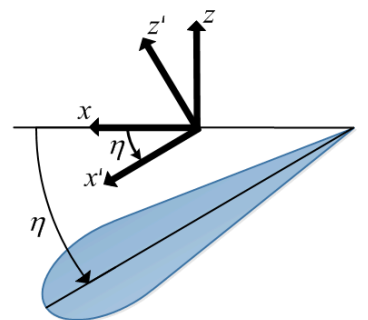

(c) Airfoil-centered view

Figure 4.1: Coordinate axes are shown in various views. Arrows point in the positive direction.

In addition to these degrees of freedom we also define the stroke plane angle $\psi$. As in Nagai's work the stroke plane is defined as the plane in which the wings undergo flapping motion [18, 17]. The stroke plane is horizontal in hovering flight and tilted at an angle $\psi$ in forward flight around the y axis of the body 
centered coordinate system.

$$
R_{\phi}(t)=\left[\begin{array}{ccc}
1 & 0 & 0 \\
0 & \cos (\phi) & \sin (\phi) \\
0 & -\sin (\phi) & \cos (\phi)
\end{array}\right], \quad R_{\lambda}(t)=\left[\begin{array}{ccc}
\cos (\lambda) & \sin (\lambda) & 0 \\
-\sin (\lambda) & \cos (\lambda) & 0 \\
0 & 0 & 1
\end{array}\right], \quad R_{\eta}(t)=\left[\begin{array}{ccc}
\cos (\eta) & 0 & -\sin (\eta) \\
0 & 1 & 0 \\
\sin (\eta) & 0 & \cos (\eta)
\end{array}\right]
$$

To facilitate a biomimetic motion we are interested in the three waveforms shown in Fig. 4.2: the sine, triangle, and trapezoidal waveforms for the heaving, sweep, and pitch, respectively. When combined these waveforms can produce patterns that roughly approximate the wing patterns of various insects including the fruit fly $[8,33]$. As in Dickinson and Sane's work, to fully define the trapezoidal waveform we prescribe a flip duration $\Delta \tau$, the time it takes for the wing to flip, and a flip start $\tau_{0}$, the point in time that the flip begins, with both expressed as a fraction of wingbeat period [23]. We relate these to the quantities used by Nagai so that the rotational acceleration time, rotational phase duration, and reversal time are $\tau_{a}=\tau_{r} / 4, \tau_{r}=0.3$, and $\tau_{t}=0.2$. respectively.
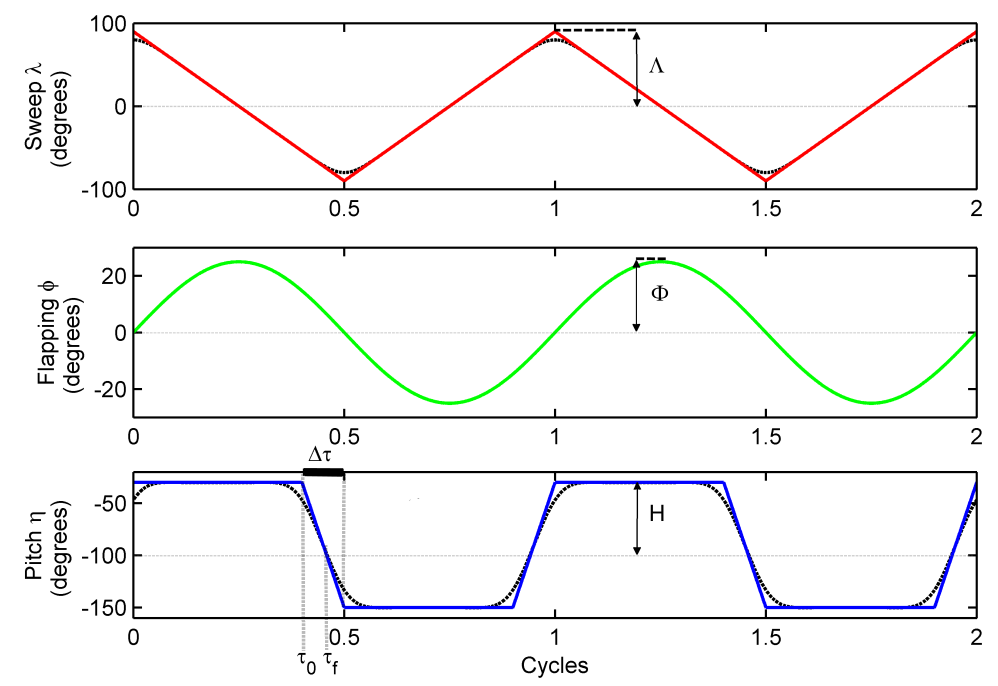

Figure 4.2: The three waveforms chosen for the wing kinematics. Solid lines are the base waveforms. Rounded lines are the smoothed waveforms. 
A reference velocity for the wing motion $V_{0}$ is taken from Nagai's referenced works and is expressed as

$$
V_{0}=2 \pi f \Lambda l_{r}
$$

where $l_{r}$ is the length from the flapping axis to the reference chord. In this case the reference chord is taken to be roughly at $2 / 3$ the wing length, giving us $l_{r}=90.7 \mathrm{~mm}$. With this we can define the advance ratio as

$$
J=U_{\infty} / V_{0}
$$

The advance ratio $J$ being one of our variables of interest for generating our parametric studies.

A more in-depth treatment of the wing kinematics and associated terms can be found in the previous works of Gomez, Bryant, and Garcia [10], and Nagai and Hayase [18].

\subsubsection{Aerodynamic Modeling}

For our aerodynamic modeling we employ the combined model developed by Gomez, Bryant, and Garcia [10]. The aerodynamic forces per unit span generated by a moving airfoil as expressed by this model are

$$
F_{x^{\prime}}=-\left(m+m_{22}\right) \dot{\eta} v_{z^{\prime}}-\rho_{\infty} \Gamma v_{z^{\prime}}-m_{11} v_{x^{\prime}}-F_{x^{\prime}}^{v}
$$




$$
F_{z^{\prime}}=\left(m+m_{11}\right) \dot{\eta} v_{x^{\prime}}+\rho_{\infty} \Gamma v_{x^{\prime}}-m_{22} \dot{v_{z^{\prime}}}-F_{z^{\prime}}^{v}
$$

where the first term in each equation accounts for the effects of calculating forces from a rotating reference frame, the second term circulation effects, the third term added mass effects, and the last term viscous effects. The circulation term is modified to include two terms that quantify the effects of rotational lift and dynamic stall, respectively.

The rotational lift concept we use comes from Anderson, Pesavento, and Wang's rotational lift based aerodynamic model [1]. This quasi-steady approach assumes the aerodynamic forces generated by an airfoil are due to its simultaneous heaving (translating) and pitching (rotating) motions in two dimensions. Here we assume the aerodynamic forces arise from two seperate sources: translational (circulatory) lift $C_{L s}$ generated by heaving and rotational lift $C_{R}$ generated by pitching.

The second term quantifies dynamic stall effects and comes from the model by Bryant, Gomez, and Garcia [4]. Dynamic stall arises from leading edge vortices (LEVs) that temporarily increase lift properties while attached to the upper wing surface. This lift enhancing phenomenon was first observed experimentally in bird and insect flight $[33,9,30,31]$, and has lead to studies conducted with mechanical models $[6,2,12,15]$.

The dynamic stall term is governed by the second order differential equation shown in Eq. 4.6. In it's original incarnation, the ODE's empirical coefficients $s_{1}$ and $s_{2}$ were fit to experimental data in the absence of rotational lift effects [4]. For use in the current combined model this ODE has been updated so that $b$ now represents the full chord instead of the half-chord, and the empirical coef- 
ficient $s_{1}$ which governs the damping ratio has been multiplied by a factor of 2 to account for rotational lift effects.

$$
\ddot{C}_{L d}+\frac{U_{c}}{b} s_{1} \dot{C}_{L d}+\frac{U_{c}^{2}}{b^{2}} s_{2} C_{L d}=\frac{U_{c}}{b} \frac{\dot{C}_{L s}}{2}
$$

With this update to the dynamic stall ODE, we now represent the circulation of our combined model as

$$
\Gamma=\frac{1}{2} C_{L s} c \sqrt{v_{x^{\prime}}^{2}+v_{z^{\prime}}^{2}}+s_{d} \frac{1}{2} C_{L d} c \sqrt{v_{x^{\prime}}^{2}+v_{z^{\prime}}^{2}}-s_{r} C_{R} c^{2} \dot{\eta}
$$

where the first term corresponds to translation from classical aerodynamic theory, second dynamic stall, and third rotational lift. This combined approach is called the BGG (Bryant-Gomez-Garcia) model. The newly introduced coefficients $s_{d}$ and $s_{r}$ act as influence factors and determine the extent to which the corresponding phenomenon is expressed in the circulation term, with both being set to 1 for the present study. This choice is supported by studies done across a number of Reynolds number ranges indicating a certain level of Re insensitivity with respect to LEV formation, with only slight differences from Re 100-1000, and nearly indistinguishable force and LEV formation from Re 2000-10000 [20].

The forces calculated by the above equations are for individual airfoil cross sections, and are integrated over the wingspan to get the overall force profile of the wing. In particular for the present study we calculate the average force generated over one flapping cycle, here called the stroke-averaged lift, by integrating the lift and drag over one flapping period.

All other terms and expressions are the same as in Bryant, Gomez, and Garcia's previous works [10, 4]. For an in-depth discussion on the combined model, 
as well as dynamic stall and rotational lift, please see the previous references.

\subsection{Results and Discussion}

\subsubsection{Verification of Numerical Model}

We begin our analysis by comparing the BGG model to the experimental data of an ornithopter in a forward flight configuration by Nagai and Hayase [18]. We will analyze the instantaneous lift and thrust generated over one flapping cycle, and then move on to the stroke averaged forces.

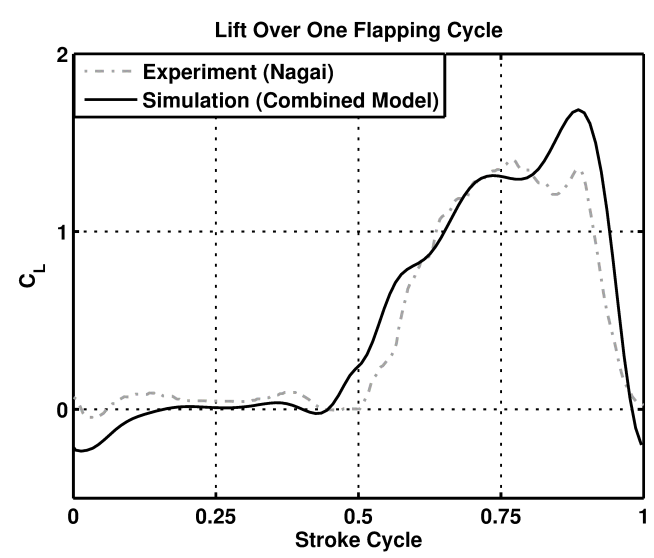

(a) Lift

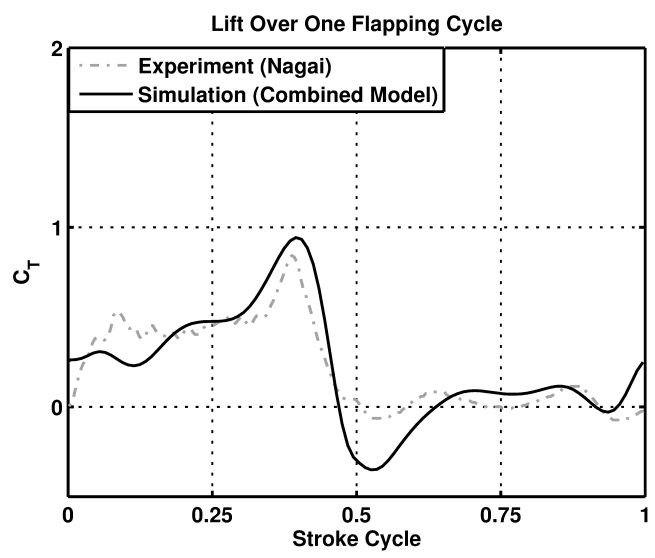

(b) Thrust

Figure 4.3: Lift and thrust for $\psi=45^{\circ}$ and $J=0.2$.

In Nagai and Hayase's work the flapping and pitching amplitudes were fixed at $60^{\circ}$ and $45^{\circ}$ respectively, while individual experiments were run for stroke plane angles of $15^{\circ}, 30^{\circ}, 45^{\circ}, 60^{\circ}$, and $75^{\circ}$. These sets of experiments were conducted for advance ratio values of $J=0.2-0.6$, forming a parametric space with which we can analyze the behavior of the combined model. 


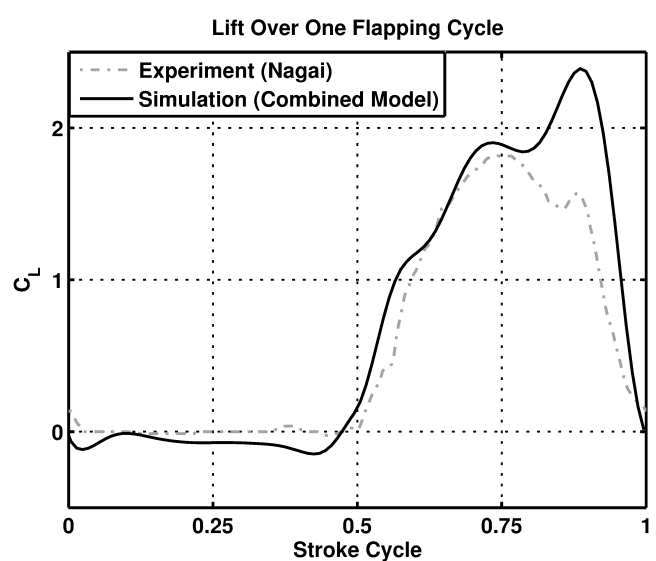

(a) Lift

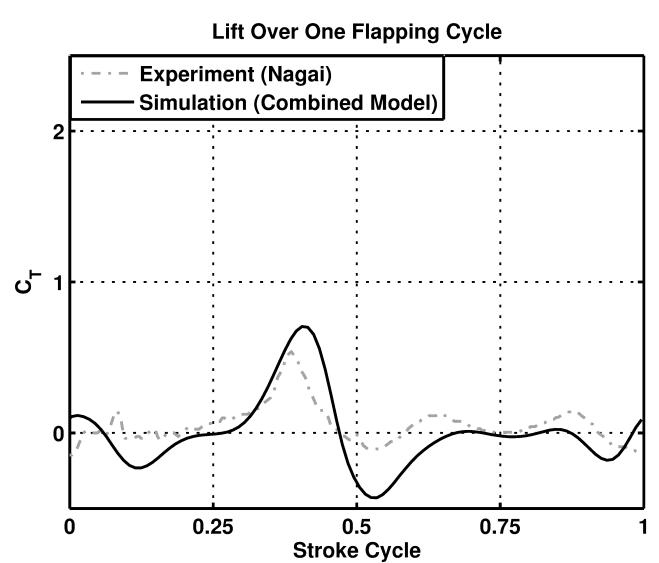

(b) Thrust

Figure 4.4: Instantaneous lift and thrust for $\psi=45^{\circ}$ and $J=0.47$.

The data for these experiments show a predictable and consistant trend of increasing total thrust and decreasing total lift as stroke plane angle increases. The combined model matches very well with all experimental data and shows the same predictable trends of increasing thrust and decreasing lift across the varying parameters. This can be seen in Fig. 4.3 and 4.4 where we have reproduced the experimental lift and drag data for $\psi=45^{\circ}$ and overlayed the numerical data results for an example comparison. These figures show a close match with respect to the instantaneous forces, a characteristic that holds true for all of the other experimental data not shown here.

There are however slight discrepencies that arise in particular parts of the force curves. To better understand the roles that dynamic stall and rotational lift play in these discrepencies it is helpful to compare these components of the combined model directly with the corresponding quantities in the experimental data. We unfortunately do not have such a breakdown of the experimental data. However, we can proceed by making an assumption about the non dynamic stall and non rotational lift components of the combined model. We 


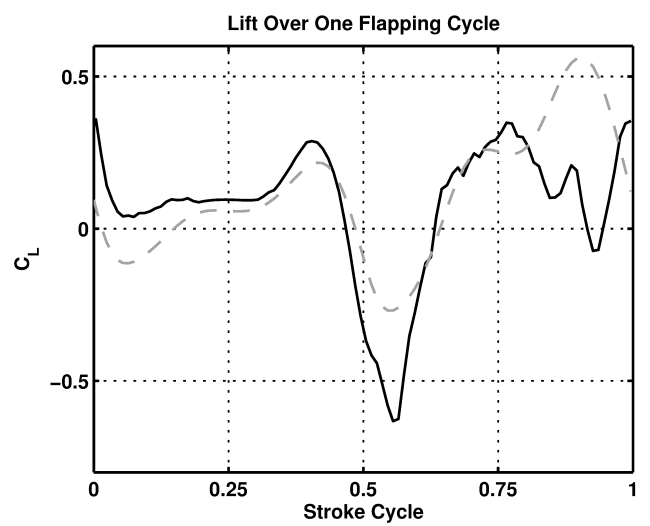

(a) Lift

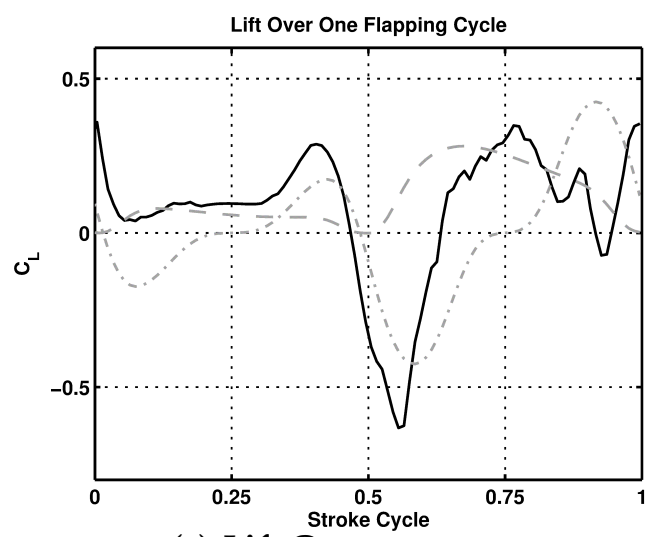

(c) Lift Components

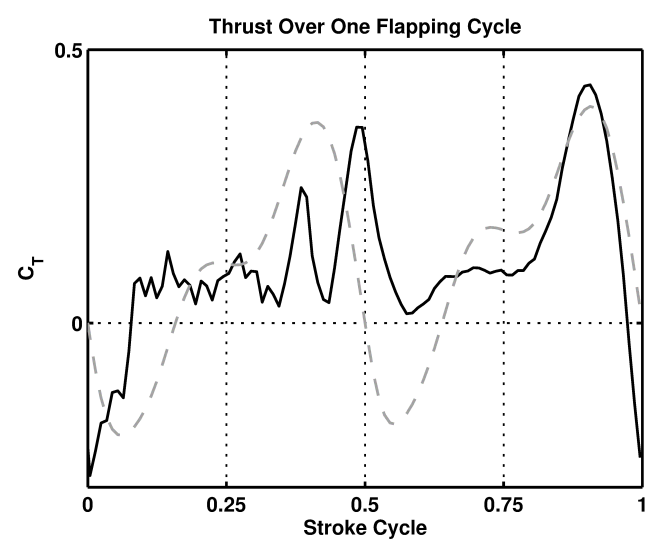

(b) Thrust

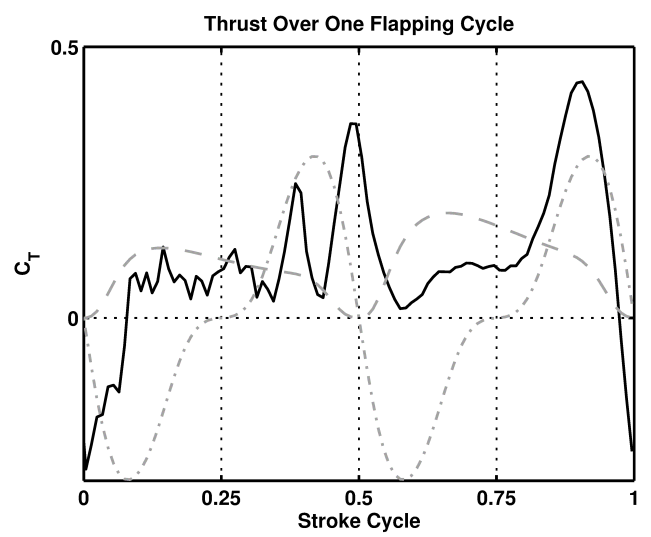

(d) Thrust Components

Figure 4.5: Experimental data minus forces not associated with Dynamic Stall and Rotational Lift for $J=0.2$.

know that these terms, such as viscous and added mass effects, are much better understood and thus can assume with reasonable certainty that they are being accurately modeled. This assumption allows us to subtract these numerical quantities directly from the experimental data, leaving us with a reasonable approximation of the quantities in the experimental data directly associated with dynamic stall and rotational lift.

This direct comparison of dynamic stall and rotational lift components is plotted in Fig. 4.5 and 4.6. The force curves in Fig. 4.5 are derived from the full experimental data in Fig. 4.3, and Fig. 4.6 from the experimental data in Fig. 4.4. 


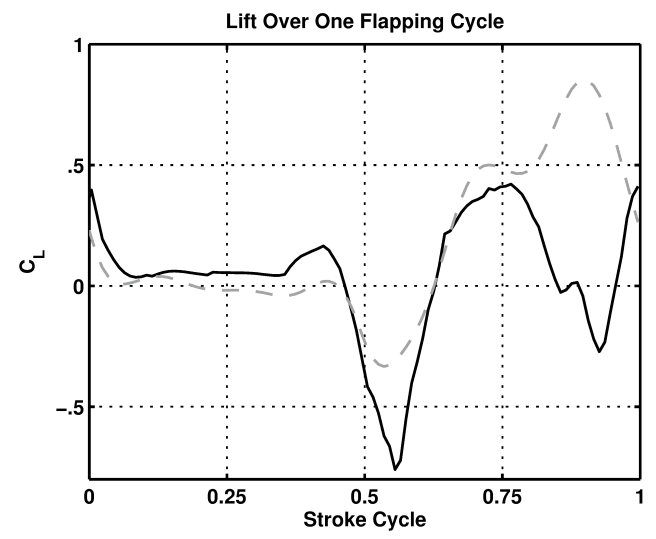

(a) Lift

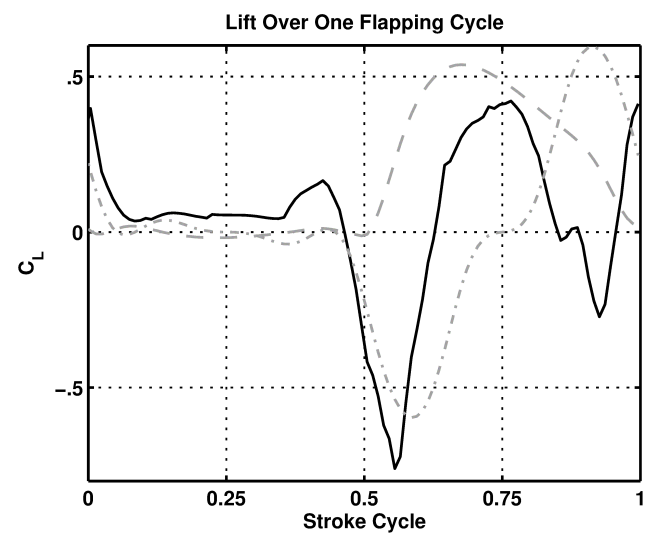

(c) Lift Components

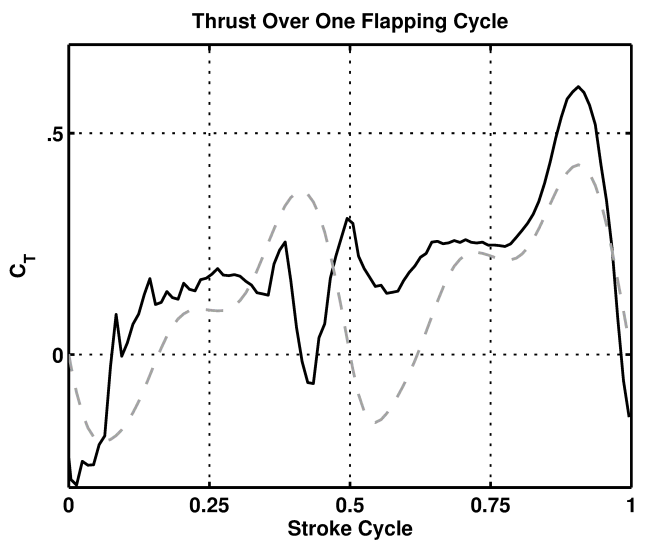

(b) Thrust

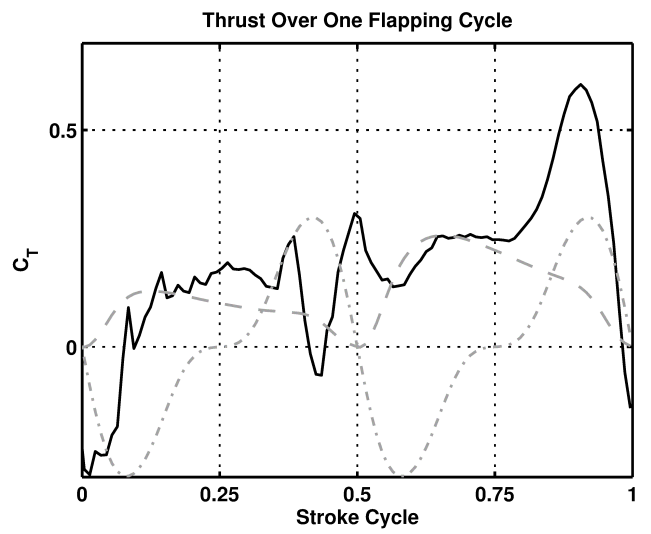

(d) Thrust Components

Figure 4.6: Experimental data minus forces not associated with Dynamic Stall and Rotational Lift for $J=0.47$.

For each sets of figures, in subfigures (a) and (b) the solid black line reprsents experimental data and the grey dashed line represents the combined numerical data for dynamic stall and rotational lift components. In subfigures (c) and (d) the grey dashed line represents the isolated numerical dynamic stall component and the grey dot-dashed line represents the isolated numerical rotational lift component.

Several important observations can be made from these figures. First, in Fig. 4.5(a), 4.5(b), and Fig. 4.6(a), 4.6(b) we can see that the discrepencies between 
numerical and experimental data match up exactly with the discrepencies seen in the full data sets from Fig. 4.3 and 4.4. This gives us confidence in the validity of the assumptions that went into making these figures, namely that the non rotational lift and non dynamic stall components are being accurately modeled, and that the components of interest are being properly teased out of the experimental data.

In addition, we can more clearly observe where these discrepencies between numerical and experimental data arise from. In Fig. 4.5(c) we can see in the second half of the stroke cycle the contributions from dynamic stall and rotational lift are offset in time slightly from the salient features in the experimental data, with the peaks and troughs from the numerical data occuring slightly ahead of that seen in the experimental data. We can see that this offset in time directly leads to the discrepency in the force curves of Fig. 4.5(a). The thrust on the other hand is not as straight forward, with the rotational lift and dynamic stall components also over and undershooting what is seen in the experimental data at different points, particularly just past the stroke cycles halfway point.

The second important observation can be gathered from Fig. 4.5(c), 4.5(d), and Fig. 4.6(c), 4.6(d). In these figures we can see that dynamic stall and rotational lift both cover seperate, but equally important, salient features of the experimental data. This again adds validity to our approach, specifically of using both components in a combined model to properly model forward flight aerodynamics within the confines of our experimental data set. Although more research is needed to understand how generally this approach holds true for flapping wing aerodynamic modeling, it is an important observation moving forward. 


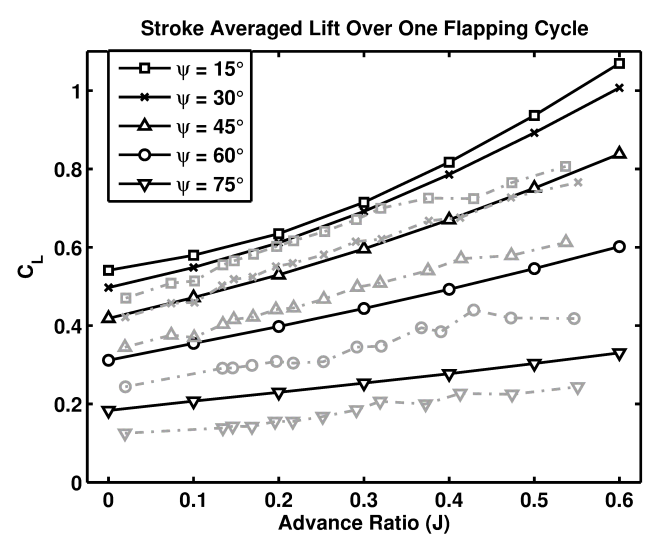

(a) Lift

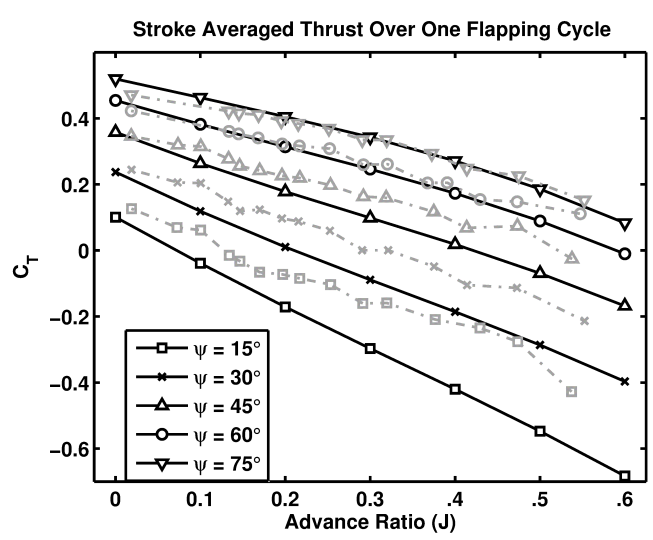

(b) Thrust

Figure 4.7: Stroke-averaged lift and thrust for all experimental data points.

The numerical and experimental data for the stroke-averaged forces are shown in Fig. 4.7. The black solid lines correspond to experimental data and grey dot-dashed lines to numerical data. As with the instantaneous forces of Fig. 4.3 and 4.4 we see a good match throughout all of the available data. The stroke-averaged lift and thrust behavior as stroke plane angle $\psi$ increases are the same in both the numerical and experimental data, corroborating previous statements. In addition the behavior seen as advance ratio varies also exhibits a close match between experimental and numerical data, with lift increasing and thrust decreasing as advance ratio increases.

This comparative analysis has shown that with respect to both the instantaneous and stroke-averaged forces the combined model does a faithful job of matching what is seen in the experimental data, justifying our choice of numerical models. 


\subsubsection{Observations of Physical Mechanisms}

The stroke-averaged lift and thrust changing over the experimental parameter space is shown in Fig. 4.8(a) and 4.8(b), respectively. Looking at the two figures as a whole we see that lift and thrust have antithetical behaviors, with lift decreasing and thrust increasing as stroke plane angle increases and the opposite occuring as advance ratio increases. This leads to the peaks and dips of the lift and drag profiles being in opposing areas of the parametric space, with the peak in lift and nadir in thrust at an advance ratio of 0.6 and stroke plane angle of $15^{\circ}$, and the peak in thrust and nadir in lift at an advance ratio of 0 and stroke plane angle of $75^{\circ}$.

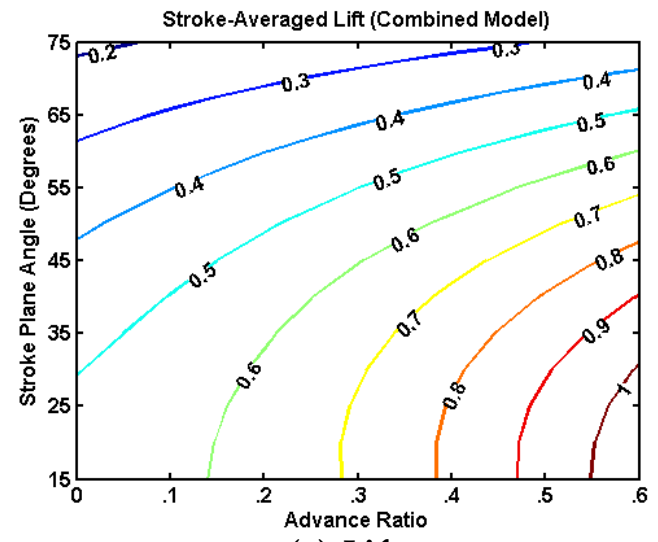

(a) Lift

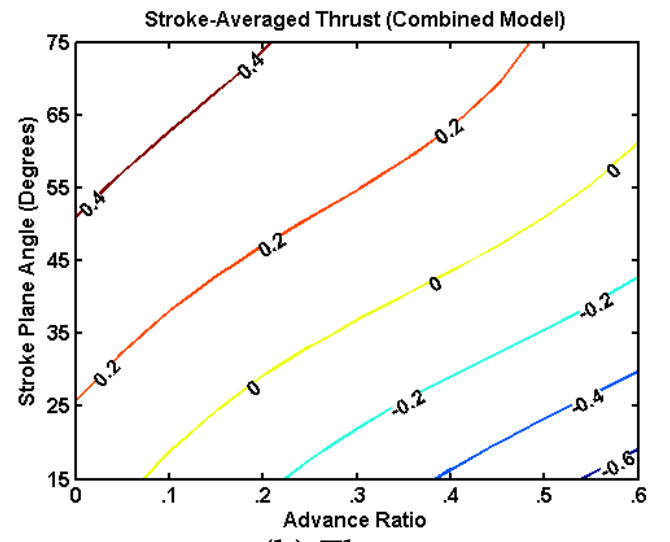

(b) Thrust

Figure 4.8: Stroke-averaged forces for $\psi=15^{\circ}-75^{\circ}$ and $J=0-0.6$.

When the stroke plane angle is zero our flapping wings are in the thovering configuration' and the aerodynamic force vector is purely in the lift direction. As the stroke plane angle increases this aerodynamic force vector shifts from the lift to the thrust direction, explaining the general decrease in lift and increase in thrust as stroke plane angle increases. 
When holding the wing kinematics constant an increase in advance ratio is caused by an increase in $U_{\infty}$. In addtion, within the experimental parameter space the magnitude of $U_{\infty}$ never approaches that of $V_{0}\left(U_{\infty}\right.$ and $V_{0}$ are equal when $J=1$ ), resulting in a net increase in lift as advance ratio increases. This may not be the result when $U_{\infty}$ is comparable to or exceeds $V_{0}$ as this would mean the ambient fluid velocity is comparable to or has exceeded the reference velocity of the wings, a kinematic area that exists outside of our current experimental parameter space.

The stroke-averaged thrust spans through negative and positive values as can be seen in Fig. 4.8(b). A boundary where net thrust equals zero exists roughly between the points $J=0.15, \psi=15^{\circ}$ and $J=.6, \psi=60^{\circ}$. Looking again at Fig. 4.8(b), above and to the left of this boundary thrust is positive, while below and to the right thrust is negative. This zero thrust boundary across the parameter space is where the drag from the ambient fluid velocity $U_{\infty}$ has balanced out the thrust produced from the flapping wings, resulting in zero net thrust on our system and a constant velocity in the thrust direction. To either side of the zero thrust boundary we have a net force acting on our system, resulting in a positve or negative acceleration depending on which side we are on. This analysis is important to begin to understand how wing kinematics affect forward flight behavior as we can now see where steady state forward flight is possible, as well as a possible way to invoke acceleration and deccelaration on our system.

The isolated contributions from dynamic stall and rotational lift are shown in Fig. 4.9 and 4.10. The contribution from rotational lift is very small, an order of magnitude smaller than dynamic stall. Dynamic stall on the other hand has 


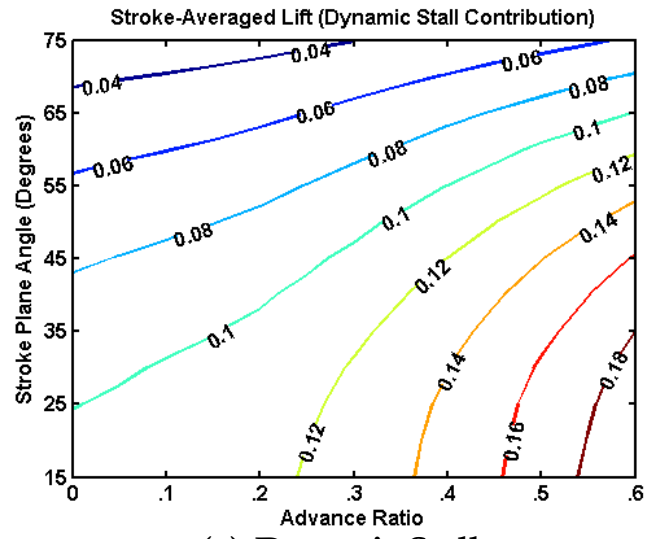

(a) Dynamic Stall

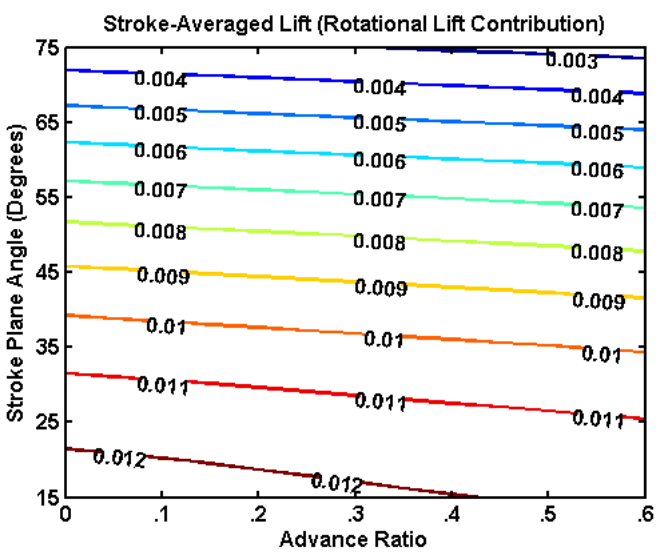

(b) Rotational Lift

Figure 4.9: Isolated contributions to stroke-averaged lift for $\psi=15^{\circ}-75^{\circ}$ and $J=0-0.6$.

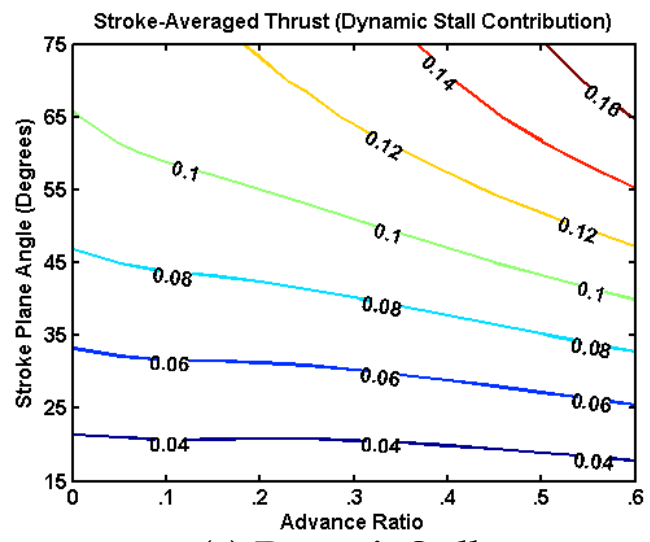

(a) Dynamic Stall

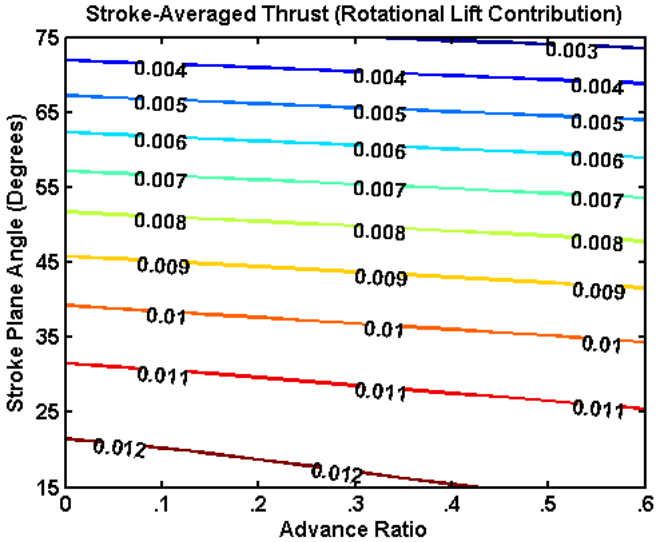

(b) Rotational Lift

Figure 4.10: Isolated contributions to stroke-averaged thrust for $\psi=15^{\circ}-$ $75^{\circ}$ and $J=0-0.6$.

a much more significant contribution with trends in the parametric space that follow that seen in the overall lift and thrust. This shows that the overall trends and main contributing factor to total lift is due to dynamic stall. 


\subsubsection{Pitch and Stroke Amplitude Effects}

We now expand our parametric study by varying the pitching amplitude $H=$ $10-90^{\circ}$. This is done for two values of the stroke amplitude; $\Lambda=45^{\circ}$ and $\Lambda=90^{\circ}$, with results shown in Fig. 4.13 and 4.14, respectively.

Looking at the $\Lambda=45^{\circ}$ case in Fig. 4.13 we see peak stroke-averaged lift occuring at $J=0.6$. This point shifts from $\psi=45^{\circ}-15^{\circ}$ as $H$ varies $10^{\circ}-$ $90^{\circ}$. This shifting of the peak lift is due primarily to a changing effective angle of attack that the wings experience as $\psi$ varies. The change in angle of attack impacts the angle of the primary force vector of the wings, causing the shift in peak lift region.

There are two points of interest with respect to the stroke-averaged thrust; peak positive thrust and peak negative thrust. Peak positive thrust remains at the same point in the parametric space, $J=0, \psi=75^{\circ}$. Peak negative thrust on the other hand occurs on the other side of the parametric space at $J=0.6$, and shifts from $\psi=15^{\circ}-75^{\circ}$ as $H$ varies $10^{\circ}-90^{\circ}$. This shows the advance ratio having the most prominent impact on stroke averaged thrust, with stroke plane angle having no influence on peak positive thrust and an influence on peak negative thrust more noticeable at greater values. These behaviors also hold true for the $\Lambda=90^{\circ}$ case depicted in Fig. 4.14.

The only significant discrepencies that arise between the $\Lambda=45^{\circ}$ and $\Lambda=90^{\circ}$ cases are in the force magnitudes. To better understand these descrepencies we analyze the changes in the peak stroke-averaged forces over the extended parametric spaces. The peak stroke-averaged lift as a function of pitch amplitude is plotted in Fig. 4.11 and the peak stroke-averaged positive and neg- 


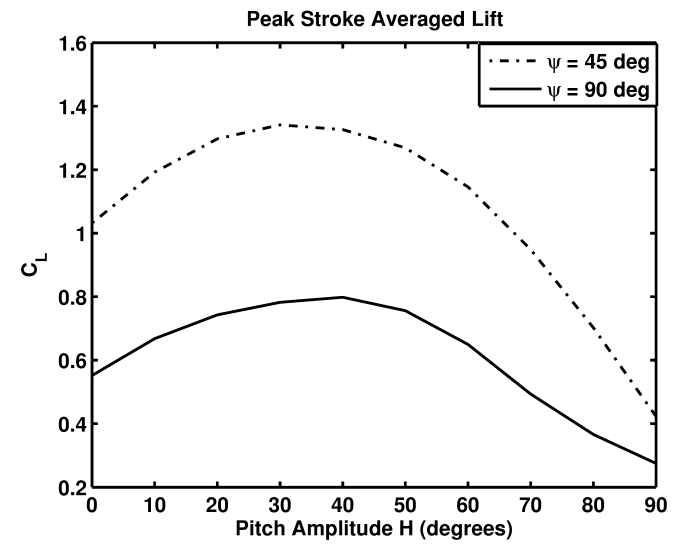

Figure 4.11: Peak stroke-averaged lift as pitch amplitude changes.

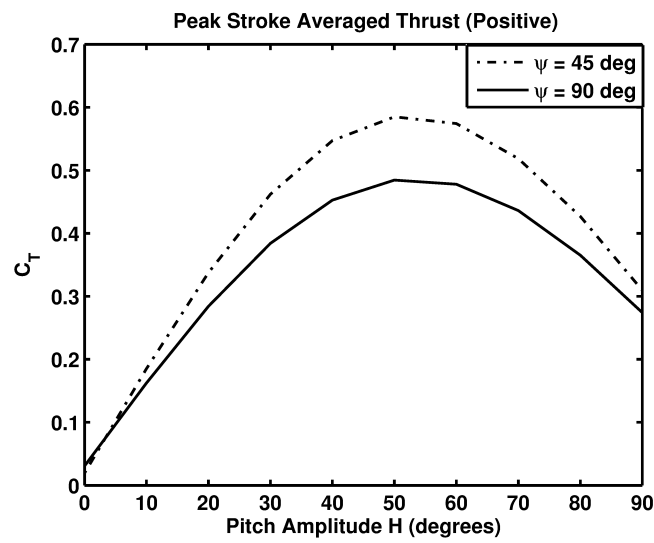

(a) Peak Positive Thrust

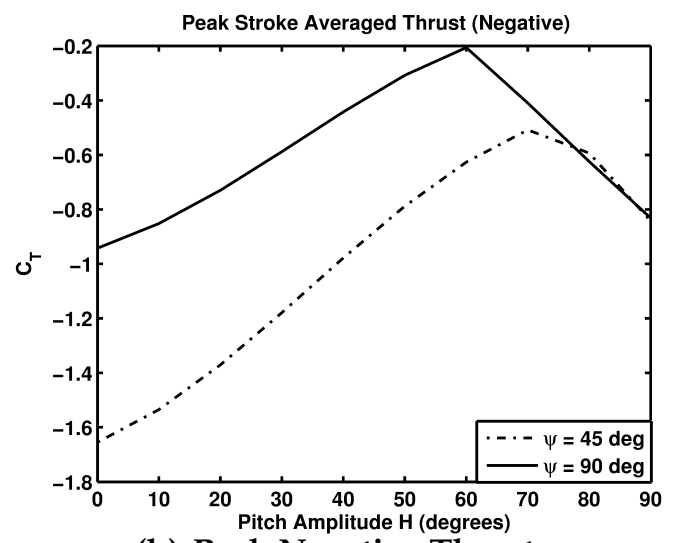

(b) Peak Negative Thrust

Figure 4.12: Peak stroke-averaged thrust as pitch amplitude changes.

ative thrust is plotted in Fig. 4.12(a) and 4.12(b), respectively. In Fig. 4.11 we see that the peak lift is significantly higher for $\Lambda=45^{\circ}$, with the maximum peak lift almost twice the magnitude of the maximum peak lift for $\Lambda=90^{\circ}$. A shift is also seen in the pitch amplitude where maximum peak lift occurs from $H=30^{\circ}$ to $40^{\circ}$ for $\Lambda=45^{\circ}$ to $90^{\circ}$.

The peak stroke-averaged negative and positive thrust also vary in magnitude between the two $\Lambda$ cases. The peak negative thrust for $\Lambda=90^{\circ}$ is larger than for $\Lambda=45^{\circ}$, with a significant difference at $H=0^{\circ}$ and tapering until the 
values converge around $H=80^{\circ}$. The maximum peak thrust again has roughly a two fold magnitude difference with the $\Lambda=90^{\circ}$ case being larger, opposite of what is seen in the stroke-averaged lift. The location of maximum peak negative thrust is shifted from the maximum peak lift to $\Lambda=60^{\circ}$ and $70^{\circ}$.

The peak stroke-averaged positive thrust on the other hand shows significantly closer magnitude between the two $\Lambda$ cases. Similar to peak lift, $\Lambda=45^{\circ}$ produces slightly higher peak positive thrust with maximum positive thrust located at $H=50^{\circ}$ for both cases.

It should also be noted that the maximum peak lift and maximum peak negative thrust occurs at $J=0.6$, and maximum peak positive thrust occurs at $J=0$. This indicates that one must be careful when seeking maximum lift output as the negative thrust, which can be unwanted and in these cases sought to be minimized, has the same tendencies to increase as advance ratio increases.

\subsection{Conclusions}

The combined low-order phenomenological model incorporating dynamic stall and rotational lift effects has been used to explore the parametric space of flapping wings in a forward flight configuration. Experimental data found in the literature was used as the basis for the initial parametric space. The changes in stroke-averaged lift and thrust around this parametric space were studied as stroke plane angle and advance ratio were varied. The lift and thrust have antithetical behaviors, with lift and thrust increasing and decreasing at opposite

points in the parametric space. Specifically, stroke-averaged lift decreases and thrust increases as stroke plane angle increases and the opposite occurring as 
advance ratio increases. Dynamic stall is also seen to have a much more significant contribution to the behavior of the overall forces produced when compared to rotational lift.

Building on the above parameter space we also vary the pitching amplitude $10^{\circ}-90^{\circ}$ for two stroke amplitude cases; $\Lambda=45^{\circ}$ and $\Lambda=90^{\circ}$. The area of peak lift and peak negative thrust shift from $\psi=45^{\circ}-15^{\circ}$ and $\psi=15^{\circ}-75^{\circ}$, respectively, as $H$ varies $10^{\circ}-90^{\circ}$ with a constant advance ratio of 0.6. Peak positive thrust on the other hand remains at a fixed advance ratio of $J=0$ and stroke plane angle $\psi=75^{\circ}$, a point in our parametric space almost antithetical to where the peak lift and negative thrust resides. These behaviors are the same for both $\Lambda$ cases, with the only significant variation seen in the force magnitudes.

This study is an example of using a combined low-order model in numerical frameworks such as optimization and, such as in this case, parametric studies. This helps round out our initial study of ornithopter flight from an overall stroke-averaged force generation stand point. Further work must be done to develop these tools for application that benifit from the low computational cost and flexibility of the low-order model. Applications such as mission specific design studies and flight path planning for example. 


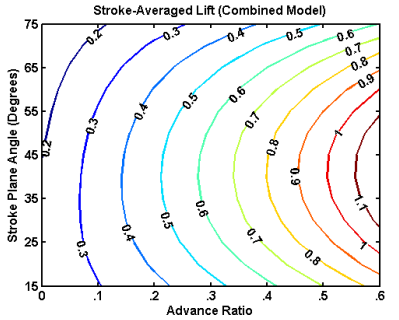

(a) Lift for $H=10^{\circ}$

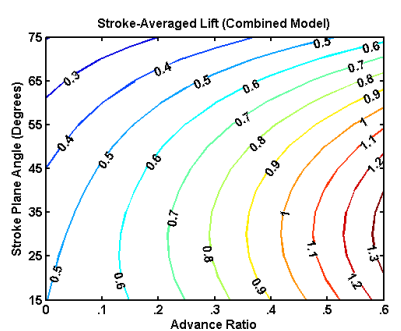

(c) Lift for $H=30^{\circ}$

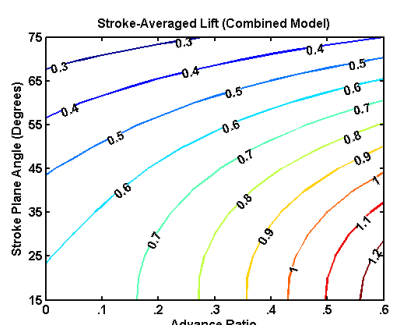

(e) Lift for $H=50^{\circ}$

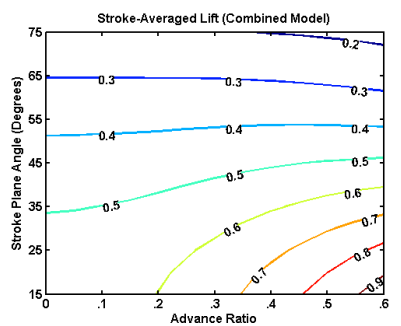

(g) Lift for $H=70^{\circ}$

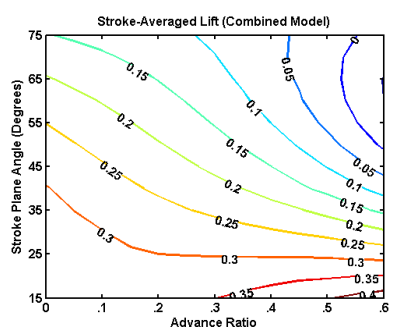

(i) Lift for $H=90^{\circ}$

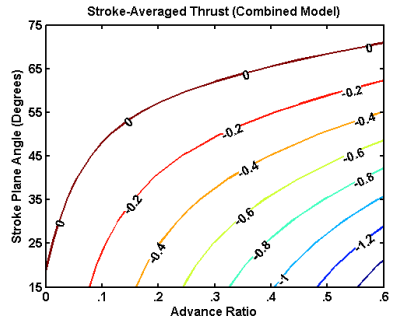

(b) Thrust for $H=10^{\circ}$

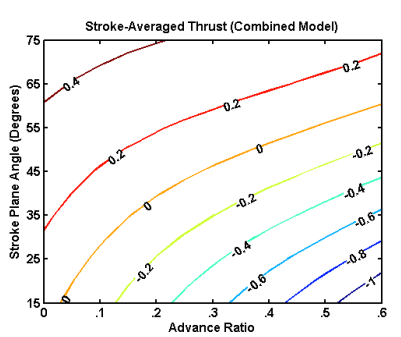

(d) Thrust for $H=30^{\circ}$

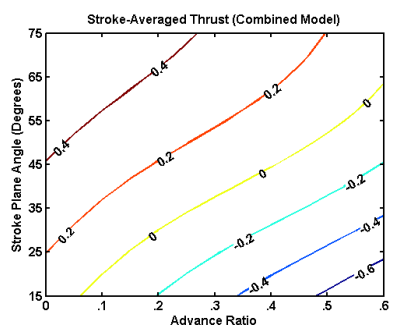

(f) Thrust for $H=50^{\circ}$

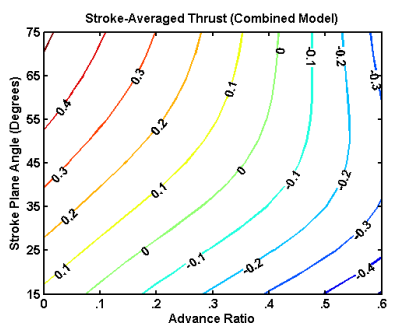

(h) Thrust for $H=70^{\circ}$

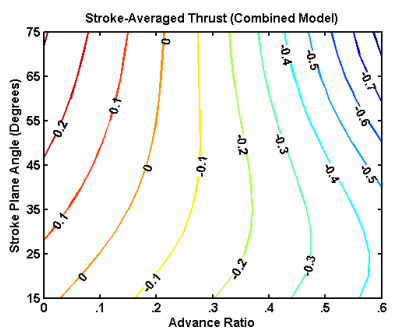

(j) Thrust for $H=90^{\circ}$

Figure 4.13: Stroke averaged forces for $\Lambda=45^{\circ}$ and $H=10^{\circ}-90^{\circ}$. 


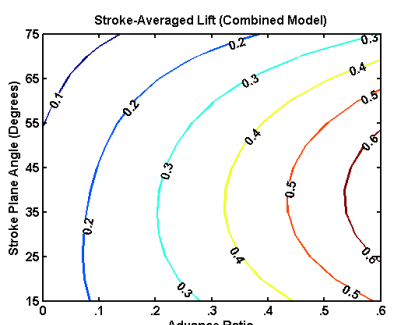

(a) Lift for $H=10^{\circ}$

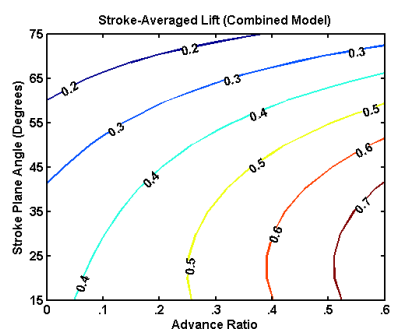

(c) Lift for $H=30^{\circ}$

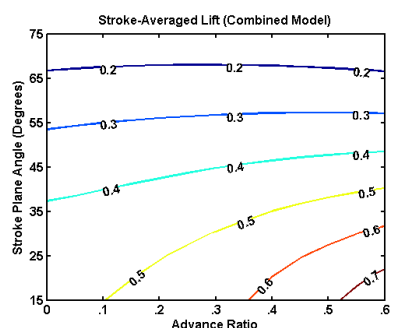

(e) Lift for $H=50^{\circ}$

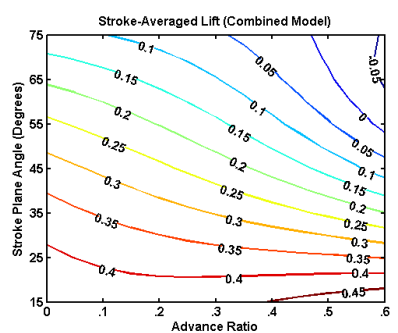

(g) $\operatorname{Lift}$ for $H=70^{\circ}$

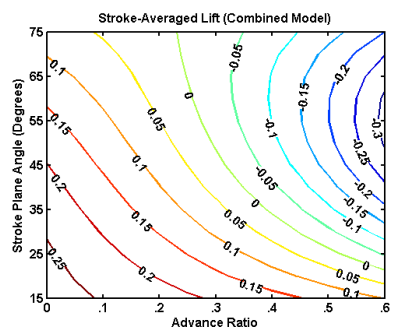

(i) Lift for $H=90^{\circ}$

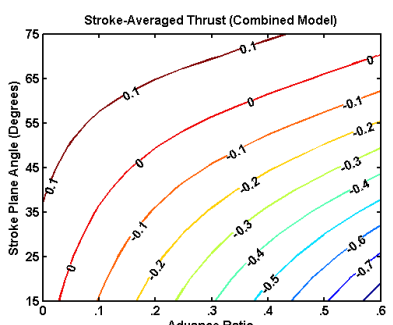

(b) Thrust for $H=10^{\circ}$

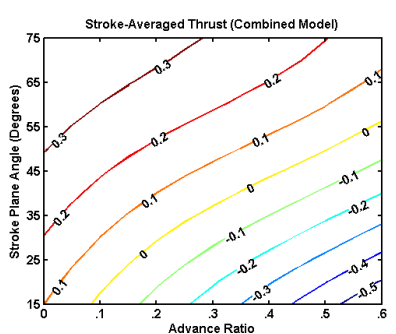

(d) Thrust for $H=30^{\circ}$

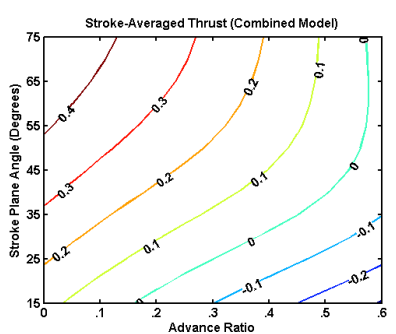

(f) Thrust for $H=50^{\circ}$

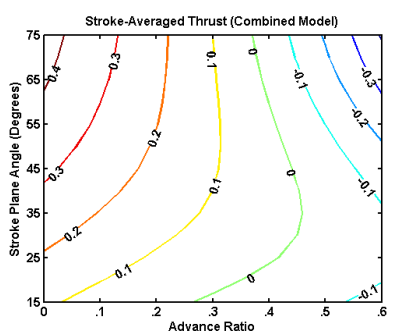

(h) Thrust for $H=70^{\circ}$

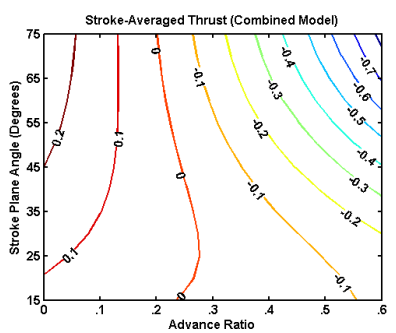

(j) Thrust for $H=90^{\circ}$

Figure 4.14: Stroke-averaged forces for $\Lambda=90^{\circ}$ and $H=10^{\circ}-90^{\circ}$. 


\section{CHAPTER 5}

\section{CONCLUSIONS}

The unique attributes and applicability of UAVs to a wide range of roles has driven their increasing ubiquity in modern society. Some of these applications may benefit from the potential flight capabilities of UAVs that exist outside of the flight envelope of current fixed wing aircraft. Ornithopter flight enables these flight capabilites by presenting a host of unsteady aerodynamic phenomena that are not present in classical fixed wing aerodynamics.

The central role UAVs occupy in ornithopter and unsteady aerodynamics research is also guiding these efforts by demanding design studies around flapping-wing UAVs to explore their capabilities and help guide the design of ornithopters optimized for specific applications.

Low-order numerical modeling takes a phenomenological approach that is informed by the system being analyzed and focuses on only capturing the more important, governing aspects of a given problem. This approach inherently has low associated computational costs that is ideal for the purposes of design and optimization studies.

The impetus for this body of work is to evaluate the capacity of low-order modeling techniques, particularly a 2D low-order model formulated from limited 2D data, to capture the salient features of the forces generated by a more

complex system, namely 3D flapping wings. The potential of these low-order models to characterize a large parameter space is also demonstrated.

Chapter 2 focused on analyzing quasi-steady rotational lift and unsteady dynamic stall. Under the kinematics analyzed the dynamic stall and rotational 
lift based models each encompass certain aspects of the salient features of the lift and drag seen in experimental data. Each approach has its own respective influencing factors that lead to differing behaviors and results in different characteristics of the experimental data being expressed in each of the approaches. Combining concepts offers a feasible approach and leads to the development of a combined model.

In chapter 3 we characterize the parametric space of an ornithopter in a hovering flight configuration using the low-order, combined model established in chapter 2. The same experimental data is used to guide the choice of parametric space where pitch and sweep are varied to characterize how the stroke-averaged lift changes with respect to these variables. The dynamic stall phenomenon was seen to have a more significant contribution to the forces produced where the total lift increases and then peaks as we approach $H=50^{\circ}$ and $\Lambda=45^{\circ}$. Expanding our parametric space to explore varying flapping frequencies reveals that the combined model has frequency independence, applying to the contributions from dynamic stall and rotational lift as well.

In chapter 4 we characterize the parametric space of an ornithopter in a forward flight configuration. In the same vein as chapter 2, here we investigated the stroke-averaged forces to study how the combined model compares to experiment and found that the lift and thrust have antithetical behaviors, with stroke-averaged lift decreasing and thrust increasing as stroke plane angle increases and the opposite occurring as advance ratio increases. As in the hovering configurtion, dynamic stall is again seen to have a much more significant contribution to the behavior of the overall forces produced. When we expand the parameter space to also include varing the pitching amplitude $10^{\circ}-90^{\circ}$ for 
two stroke amplitude cases, we see that the area of peak lift and peak negative thrust shift across the parameter space. The peak positive thrust on the other hand remains at a fixed location in our parametric space that is almost antithetical to where the peak lift and negative thrust resides.

This study is an example of using a combined low-order model in numerical frameworks such as, what is used here, parametric studies. This helps round out our initial study of ornithopter flight from an overall stroke-averaged force generation stand point. This body of work is a starting point to move forward with greater understanding of how to better capture unsteady phenomena with loworder modeling techniques and helps us in evaluating low-order, phenomenological modeling approaches that use existing data and techniques to model a more complex system. In particular, it helps us gain a more intimate understanding of the applicability of the combined model, as well as its capacity as a simplified model utilizing limited finite data to model a more complex threedimensional system. 


\section{BIBLIOGRAPHY}

[1] A. Andersen, U. Pesavento, and Z. Jane Wang. Unsteady aerodynamics of fluttering and tumbling plates. Journal of Fluid Mechanics, 541:65-90, October 2005.

[2] Hikaru Aono, Satish K. Chimakurthi, Pin Wu, Erik Sallstrom, Bret K. Stanford, Carlos E. S. Cesnik, Peter Ifju, Lawrence Ukeiley, and Wei Shyy. A computational and experimental study of flexible flapping wing aerodynamics. In AIAA Aerospace Sciences Meeting Including the New Horizons Forum and Aerospace Exposition, number January, pages 1-22, Orlando, Florida, 2010.

[3] Gordon J. Berman and Z. Jane Wang. Energy-minimizing kinematics in hovering insect flight. Journal of Fluid Mechanics, 582:153-68, June 2007.

[4] Matthew Bryant, Juan Carlos Gomez, and Ephrahim Garcia. ReducedOrder Aerodynamic Modeling of Flapping Wing Energy Harvesting at Low Reynolds Number. AIAA Journal, pages 1-12, September 2013.

[5] JD DeLaurier. An aerodynamic model for flapping-wing flight. Aeronautical Journal, 97:125-130, 1993.

[6] M. H. Dickinson, F Lehmann, and Sanjay Sane. Wing Rotation and the Aerodynamic Basis of Insect Flight. Science, 284(5422):1954-1960, June 1999.

[7] Jeff D Eldredge, Chengjie Wang, and Michael V OL. A Computational Study of a Canonical Pitch-up, Pitch-down Wing Maneuver. In 39th AIAA Fluid Dynamics Conference, number June, pages 1-14, 2009.

[8] CP Ellington. The aerodynamics of hovering insect flight. III. Kinematics. Philosophical Transactions of the Royal Society B: Biological Sciences, 305(1122):41-78, 1984.

[9] CP Ellington, C Van Den Berg, AP Willmott, and Adrian L. R. Thomas. Leading-edge vortices in insect flight. Letters To Nature, 384:626-30, 1996.

[10] Juan C. Gomez, Matthew Bryant, and Ephrahim Garcia. Low-Order Modeling of the Unsteady Aerodynamics in Flapping Wings. Journal of Aircraft, pages 1-10, 2014. 
[11] Juan Carlos Gomez and Ephrahim Garcia. Morphing unmanned aerial vehicles. Smart Materials and Structures, 20(10):1-16, October 2011.

[12] S. Heathcote, Z. Wang, and I. Gursul. Effect of spanwise flexibility on flapping wing propulsion. Journal of Fluids and Structures, 24(2):183-199, February 2008.

[13] Dae-Kwan Kim, Jun-Seong Lee, Jin-Young Lee, and Jae-Hung Han. An aeroelastic analysis of a flexible flapping wing using modified strip theory. In Proceedings of SPIE, volume 6928, pages 69281O-1-69281O-8. Spie, 2008.

[14] T. Kinsey and G. Dumas. Parametric Study of an Oscillating Airfoil in a Power-Extraction Regime. AIAA Journal, 46(6):1318-1330, June 2008.

[15] K. B. Lua, T. T. Lim, and K. S. Yeo. Effect of wingwake interaction on aerodynamic force generation on a 2D flapping wing. Experiments in Fluids, 51(1):177-195, January 2011.

[16] Max M. Munk. Note On the Air Forces On a Wing Caused By Pitching. Technical report, National Advisory Committee for Aeronautics, 1925.

[17] Hiroto Nagai and Koji Isogai. Effects of Flapping Wing Kinematics on Hovering and Forward Flight Aerodynamics. AIAA Journal, 49(8):1750-1762, 2011.

[18] Hiroto Nagai, Koji Isogai, Tatsumi Fujimoto, and Toshiyuki Hayase. Experimental and Numerical Study of Forward Flight Aerodynamics of Insect Flapping Wing. AIAA Journal, 47(3):730-742, 2009.

[19] Michael V OL. The High-Frequency , High-Amplitude Pitch Problem : Airfoils, Plates and Wings. In 39th AIAA Fluid Dynamics Conference, number June, pages 1-14, 2009.

[20] Michael V OL, Jeff D Eldredge, Yongsheng Lian, Daniel J Garmann, and Aaron Altman. Résumé of the AIAA FDTC Low Reynolds Number Discussion Groups Canonical Cases. In 48th AIAA Aerospace Sciences Meeting, number January, pages 1-18, 2010.

[21] Christopher T. Orlowski and Anouck R. Girard. Modeling and Simulation of Nonlinear Dynamics of Flapping Wing Micro Air Vehicles. AIAA Journal, 49(5):969-981, May 2011. 
[22] Thomas Rakotomamonjy, Mustapha Ouladsine, and Thierry Le Moing. Modelization and Kinematics Optimization for a Flapping-Wing Microair Vehicle. Journal of Aircraft, 44(1):217-231, January 2007.

[23] S P Sane and M H Dickinson. The control of flight force by a flapping wing: lift and drag production. The Journal of experimental biology, 204(Pt 15):2607-26, August 2001.

[24] Sergey Shkarayev and Dmytro Silin. Measurements of Aerodynamic Coefficients for Flapping Wings at 0-90 Angles of Attack. AIAA Journal, 50(10):2034-2042, October 2012.

[25] Theodore Theodorsen. General Theory of Aerodynamic Instability and the Mechanism of Flutter. Technical report, National Advisory Committee for Aeronautics, 1935.

[26] C. T. Tran and D. Petot. Semi-Empirical Model for the Dynamic Stall of Airfoils in View of Application to the Calculated Responses of a Helicopter in Forward Flight. Vertica, 5(1):35-53, 1981.

[27] Marcos Vanella, Timothy Fitzgerald, Sergio Preidikman, Elias Balaras, and Balakumar Balachandran. Influence of flexibility on the aerodynamic performance of a hovering wing. The Journal of experimental biology, 212(Pt 1):95-105, January 2009.

[28] Dragos Viieru, Jian Tang, Yongsheng Lian, Hao Liu, and Wei Shyy. Flapping and Flexible Wing Aerodynamics of Low Reynolds Number Flight Vehicles. In Aerospace Sciences Meeting and Exhibit, number January, pages 1-18, Reno, Nevada, 2006.

[29] Jeffrey A. Walker. Rotational Lift: Something Different or More of the Same? The Journal of Experimental Biology, 205:3783-3792, 2002.

[30] Douglas R Warrick, Bret W Tobalske, and Donald R Powers. Lift production in the hovering hummingbird. Proceedings. Biological sciences / The Royal Society, 276(1674):3747-52, December 2009.

[31] Torkel Weis-fogh. Quick estimates of flight fitness in hovering animals, including novel mechanisms for lift production. Journal of Experimental Biology, 59:169-230, 1973.

[32] Adam M. Wickenheiser and Ephrahim Garcia. Optimization of Perching 
Maneuvers Through Vehicle Morphing. Journal of Guidance, Control, and Dynamics, 31(4):815-823, July 2008.

[33] J. M. Zanker. The Wing Beat of Drosophila Melanogaster. I. Kinematics. Philosophical Transactions of the Royal Society B: Biological Sciences, 327(1238):1-18, February 1990.

[34] Patrick Zdunich, Derek Bilyk, Marc MacMaster, David Loewen, James DeLaurier, Roy Kornbluh, Tom Low, Scott Stanford, and Dennis Holeman. Development and Testing of the Mentor Flapping-Wing Micro Air Vehicle. Journal of Aircraft, 44(5):1701-1711, September 2007.

[35] Liang Zhao, Qingfeng Huang, Xinyan Deng, and Sanjay Sane. The effect of chord-wise flexibility on the aerodynamic force generation of flapping wings: experimental studies. In IEEE International Conference on Robotics and Automation, pages 0-5, Kobe, Japan, 2009. 